\title{
Projections of Future Climate Change
}

\section{Co-ordinating Lead Authors}

U. Cubasch, G.A. Meehl

\section{Lead Authors}

G.J. Boer, R.J. Stouffer, M. Dix, A. Noda, C.A. Senior, S. Raper, K.S. Yap

\section{Contributing Authors}

A. Abe-Ouchi, S. Brinkop, M. Claussen, M. Collins, J. Evans, I. Fischer-Bruns, G. Flato, J.C. Fyfe, A. Ganopolski, J.M. Gregory, Z.-Z. Hu, F. Joos, T. Knutson, R. Knutti, C. Landsea, L. Mearns, C. Milly, J.F.B. Mitchell, T. Nozawa, H. Paeth, J. Räisänen, R. Sausen, S. Smith, T. Stocker, A. Timmermann, U. Ulbrich, A. Weaver, J. Wegner, P. Whetton, T. Wigley, M. Winton, F. Zwiers

\section{Review Editors}

J.-W. Kim, J. Stone 


\section{Contents}

Executive Summary $\quad 527$

9.1 Introduction

530

9.1.1 Background and Recap of Previous Reports 530

9.1.2 New Types of Model Experiments since 1995

9.2 Climate and Climate Change

9.2.1 Climate Forcing and Climate Response 532

9.2.2 Simulating Forced Climate Change 534

9.2.2.1 Signal versus noise 534

9.2.2.2 Ensembles and averaging $\quad 534$

9.2.2.3 Multi-model ensembles $\quad 535$

9.2.2.4 Uncertainty 536

9.3 Projections of Climate Change 536

9.3.1 Global Mean Response 536

9.3.1.1 $1 \% / \mathrm{yr} \mathrm{CO}_{2}$ increase (CMIP2) experiments

9.3.1.2 Projections of future climate from forcing scenario experiments (IS92a)

9.3.1.3 Marker scenario experiments (SRES)

9.3.2 Patterns of Future Climate Change 9.3.2.1 Summary

543

548

9.3.3 Range of Temperature Response to SRES Emission Scenarios

554
9.3.3.1 Implications for temperature of stabilisation of greenhouse gases 557

9.3.4 Factors that Contribute to the Response $\quad 559$

9.3.4.1 Climate sensitivity 559

9.3.4.2 The role of climate sensitivity and ocean heat uptake 561

9.3.4.3 Thermohaline circulation changes 562

9.3.4.4 Time-scales of response $\quad 563$

9.3.5 Changes in Variability 565

9.3.5.1 Intra-seasonal variability $\quad 566$

9.3.5.2 Interannual variability $\quad 567$

9.3.5.3 Decadal and longer time-scale variability $\quad 568$

9.3.5.4 Summary $\quad 570$

9.3.6 Changes of Extreme Events $\quad 570$

9.3.6.1 Temperature 570

9.3.6.2 Precipitation and convection $\quad 572$

9.3.6.3 Extra-tropical storms $\quad 573$

9.3.6.4 Tropical cyclones $\quad 574$

9.3.6.5 Commentary on changes in extremes of weather and climate 574

9.3.6.6 Conclusions $\quad 575$

\subsection{General Summary 576}

Appendix 9.1: Tuning of a Simple Climate Model to AOGCM Results 577

References 


\section{Executive Summary}

The results presented in this chapter are based on simulations made with global climate models and apply to spacial scales of hundreds of kilometres and larger. Chapter 10 presents results for regional models which operate on smaller spatial scales. Climate change simulations are assessed for the period 1990 to 2100 and are based on a range of scenarios for projected changes in greenhouse gas concentrations and sulphate aerosol loadings (direct effect). A few Atmosphere-Ocean General Circulation Model (AOGCM) simulations include the effects of ozone and/or indirect effects of aerosols (see Table 9.1 for details). Most integrations $^{1}$ do not include the less dominant or less well understood forcings such as land-use changes, mineral dust, black carbon, etc. (see Chapter 6). No AOGCM simulations include estimates of future changes in solar forcing or in volcanic aerosol concentrations.

There are many more AOGCM projections of future climate available than was the case for the IPCC Second Assessment Report (IPCC, 1996) (hereafter SAR). We concentrate on the IS92a and draft SRES A2 and B2 scenarios. Some indication of uncertainty in the projections can be obtained by comparing the responses among models. The range and ensemble standard deviation are used as a measure of uncertainty in modelled response. The simulations are a combination of a forced climate change component together with internally generated natural variability. A number of modelling groups have produced ensembles of simulations where the projected forcing is the same but where variations in initial conditions result in different evolutions of the natural variability. Averaging these integrations preserves the forced climate change signal while averaging out the natural variability noise, and so gives a better estimate of the models' projected climate change.

For the AOGCM experiments, the mean change and the range in global average surface air temperature (SAT) for the 1961 to 1990 average to the mid-21st century (2021 to 2050) for IS92a is $+1.3^{\circ} \mathrm{C}$ with a range from +0.8 to $+1.7^{\circ} \mathrm{C}$ for greenhouse gas plus sulphates (GS) as opposed to $+1.6^{\circ} \mathrm{C}$ with a range from +1.0 to $+2.1^{\circ} \mathrm{C}$ for greenhouse gas only (G). For SRES A2 the mean is $+1.1^{\circ} \mathrm{C}$ with a range from +0.5 to $+1.4^{\circ} \mathrm{C}$, and for $\mathrm{B} 2$, the mean is $+1.2^{\circ} \mathrm{C}$ with a range from +0.5 to $+1.7^{\circ} \mathrm{C}$.

For the end of the 21st century (2071 to 2100), for the draft SRES marker scenario A2, the global average SAT change from AOGCMs compared with 1961 to 1990 is $+3.0^{\circ} \mathrm{C}$ and the range is +1.3 to $+4.5^{\circ} \mathrm{C}$, and for $\mathrm{B} 2$ the mean SAT change is $+2.2^{\circ} \mathrm{C}$ and the range is +0.9 to $+3.4^{\circ} \mathrm{C}$.

AOGCMs can only be integrated for a limited number of scenarios due to computational expense. Therefore, a simple climate model is used here for the projections of climate change for the next century. The simple model is tuned to simulate the response found in several of the AOGCMs used here. The forcings for the simple model are based on the radiative forcing estimates from Chapter 6, and are slightly different to the forcings used by the AOGCMs. The indirect aerosol forcing is

\footnotetext{
${ }^{1}$ In this report, the term "integration" is used to mean a climate model rum.
}

scaled assuming a value of $-0.8 \mathrm{Wm}^{-2}$ for 1990 . Using the IS92 scenarios, the SAR gives a range for the global mean temperature change for 2100 , relative to 1990 , of +1 to $+3.5^{\circ} \mathrm{C}$. The estimated range for the six final illustrative SRES scenarios using updated methods is +1.4 to $+5.6^{\circ} \mathrm{C}$. The range for the full set of SRES scenarios is +1.4 to $+5.8^{\circ} \mathrm{C}$.

These estimates are larger than in the SAR, partly as a result of increases in the radiative forcing, especially the reduced estimated effects of sulphate aerosols in the second half of the 21 st century. By construction, the new range of temperature responses given above includes the climate model response uncertainty and the uncertainty of the various future scenarios, but not the uncertainty associated with the radiative forcings, particularly aerosol. Note the AOGCM ranges above are 30-year averages for a period ending at the year 2100 compared to the average for the period 1961 to 1990 , while the results for the simple model are for temperature changes at the year 2100 compared with the year 1990.

A traditional measure of climate response is equilibrium climate sensitivity derived from $2 \times \mathrm{CO}_{2}$ experiments with mixedlayer models, i.e., Atmospheric General Circulation Models (AGCMs) coupled to non-dynamic slab oceans, run to equilibrium. It has been cited historically to provide a calibration for models used in climate change experiments. The mean and standard deviation of this quantity from seventeen mixed-layer models used in the SAR are +3.8 and $+0.8^{\circ} \mathrm{C}$, respectively. The same quantities from fifteen models in active use are +3.5 and $+0.9^{\circ} \mathrm{C}$, not significantly different from the values in the SAR. These quantities are model dependent, and the previous estimated range for this quantity, widely cited as +1.5 to $+4.5^{\circ} \mathrm{C}$, still encompasses the more recent model sensitivity estimates.

A more relevant measure of transient climate change is the transient climate response (TCR). It is defined as the globally averaged surface air temperature change for AOGCMs at the time of $\mathrm{CO}_{2}$ doubling in $1 \% / \mathrm{yr} \mathrm{CO}_{2}$ increase experiments. The TCR combines elements of model sensitivity and factors that affect response (e.g., ocean heat uptake). It provides a useful measure for understanding climate system response and allows direct comparison of global coupled models. The range of TCR for current AOGCMs is +1.1 to $+3.1^{\circ} \mathrm{C}$ with an average of $1.8^{\circ} \mathrm{C}$. The $1 \% / \mathrm{yr} \mathrm{CO}_{2}$ increase represents the changes in radiative forcing due to all greenhouse gases, hence this is a higher rate than is projected for $\mathrm{CO}_{2}$ alone. This increase of radiative forcing lies on the high side of the SRES scenarios (note also that $\mathrm{CO}_{2}$ doubles around mid-21st century in most of the scenarios). However these experiments are valuable for promoting the understanding of differences in the model responses.

The following findings from the models analysed in this chapter corroborate results from the SAR (projections of regional climate change are given in Chapter 10) for all scenarios considered. We assign these to be virtually certain to very likely (defined as agreement among most models, or, where only a small number of models have been analysed and their results are physically plausible, these have been assessed to characterise those from a larger number of models). The more recent results are generally obtained from models with improved parametrizations (e.g., better land-surface process schemes). 
- The troposphere warms, stratosphere cools, and near surface temperature warms.

- Generally, the land warms faster than the ocean, the land warms more than the ocean after forcing stabilises, and there is greater relative warming at high latitudes.

- The cooling effect of tropospheric aerosols moderates warming both globally and locally, which mitigates the increase in SAT.

- The SAT increase is smaller in the North Atlantic and circumpolar Southern Ocean regions relative to the global mean.

- As the climate warms, Northern Hemisphere snow cover and sea-ice extent decrease.

- The globally averaged mean water vapour, evaporation and precipitation increase.

- Most tropical areas have increased mean precipitation, most of the sub-tropical areas have decreased mean precipitation, and in the high latitudes the mean precipitation increases.

- Intensity of rainfall events increases.

- There is a general drying of the mid-continental areas during summer (decreases in soil moisture). This is ascribed to a combination of increased temperature and potential evaporation that is not balanced by increases in precipitation.

- A majority of models show a mean El Niño-like response in the tropical Pacific, with the central and eastern equatorial Pacific sea surface temperatures warming more than the western equatorial Pacific, with a corresponding mean eastward shift of precipitation.

- Available studies indicate enhanced interannual variability of northern summer monsoon precipitation.

- With an increase in the mean surface air temperature, there are more frequent extreme high maximum temperatures and less frequent extreme low minimum temperatures. There is a decrease in diurnal temperature range in many areas, with night-time lows increasing more than daytime highs. A number of models show a general decrease in daily variability of surface air temperature in winter, and increased daily variability in summer in the Northern Hemisphere land areas.

- The multi-model ensemble signal to noise ratio is greater for surface air temperature than for precipitation.

- Most models show weakening of the Northern Hemisphere thermohaline circulation (THC), which contributes to a reduction in the surface warming in the northern North Atlantic. Even in models where the THC weakens, there is still a warming over Europe due to increased greenhouse gases.
- The deep ocean has a very long thermodynamic response time to any changes in radiative forcing; over the next century, heat anomalies penetrate to depth mainly at high latitudes where mixing is greatest.

A second category of results assessed here are those that are new since the SAR, and we ascribe these to be very likely (as defined above):

- The range of the TCR is limited by the compensation between the effective climate sensitivity (ECS) and ocean heat uptake. For instance, a large ECS, implying a large temperature change, is offset by a comparatively large heat flux into the ocean.

- Including the direct effect of sulphate aerosols (IS92a or similar) reduces global mean mid-21st century warming (though there are uncertainties involved with sulphate aerosol forcing - see Chapter 6).

- Projections of climate for the next 100 years have a large range due both to the differences of model responses and the range of emission scenarios. Choice of model makes a difference comparable to choice of scenario considered here.

- In experiments where the atmospheric greenhouse gas concentration is stabilised at twice its present day value, the North Atlantic THC recovers from initial weakening within one to several centuries.

- The increases in surface air temperature and surface absolute humidity result in even larger increases in the heat index (a measure of the combined effects of temperature and moisture). The increases in surface air temperature also result in an increase in the annual cooling degree days and a decrease in heating degree days.

Additional new results since the SAR; these are assessed to be likely due to many (but not most) models showing a given result, or a small number of models showing a physically plausible result.

- Areas of increased 20 year return values of daily maximum temperature events are largest mainly in areas where soil moisture decreases; increases in return values of daily minimum temperature especially occur over most land areas and are generally larger where snow and sea ice retreat.

- Precipitation extremes increase more than does the mean and the return period for extreme precipitation events decreases almost everywhere.

Another category includes results from a limited number of studies which are new, less certain, or unresolved, and we assess these to have medium likelihood, though they remain physically plausible: 
- Although the North Atlantic THC weakens in most models, the relative roles of surface heat and freshwater fluxes vary from model to model. Wind stress changes appear to play only a minor role.

- It appears that a collapse in the THC by the year 2100 is less likely than previously discussed in the SAR, based on the AOGCM results to date.

- Beyond 2100, the THC could completely shut-down, possibly irreversibly, in either hemisphere if the rate of change of radiative forcing was large enough and applied long enough. The implications of a complete shut-down of the THC have not been fully explored.

- Although many models show an El Niño-like change in the mean state of tropical Pacific SSTs, the cause is uncertain. It has been related to changes in the cloud radiative forcing and/or evaporative damping of the east-west SST gradient in some models.

- Future changes in El Niño-Southern Oscillation (ENSO) interannual variability differ from model to model. In models that show increases, this is related to an increase in thermocline intensity, but other models show no significant change and there are considerable uncertainties due to model limitations of simulating ENSO in the current generation of AOGCMs (Chapter 8).

- Several models produce less of the weak but more of the deeper mid-latitude lows, meaning a reduced total number of storms. Techniques are being pioneered to study the mechanisms of the changes and of variability, but general agreement among models has not been reached.

- There is some evidence that shows only small changes in the frequency of tropical cyclones derived from large-scale parameters related to tropical cyclone genesis, though some measures of intensities show increases, and some theoretical and modelling studies suggest that upper limit intensities could increase (for further discussion see Chapter 10).

- There is no clear agreement concerning the changes in frequency or structure of naturally occurring modes of variability such as the North Atlantic Oscillation. 


\subsection{Introduction}

The purpose of this chapter is to assess and quantify projections of possible future climate change from climate models. A background of concepts used to assess climate change experiments is presented in Section 9.2, followed by Section 9.3 which includes results from ensembles of several categories of future climate change experiments, factors that contribute to the response of those models, changes in variability and changes in extremes. Section 9.4 is a synthesis of our assessment of model projections of climate change.

In a departure from the organisation of the SAR, the assessment of regional information derived in some way from global models (including results from embedded regional high resolution models, downscaling, etc.) now appears in Chapter 10.

\subsubsection{Background and Recap of Previous Reports}

Studies of projections of future climate change use a hierarchy of coupled ocean/atmosphere/sea-ice/land-surface models to provide indicators of global response as well as possible regional patterns of climate change. One type of configuration in this climate model hierarchy is an Atmospheric General Circulation Model (AGCM), with equations describing the time evolution of temperature, winds, precipitation, water vapour and pressure, coupled to a simple non-dynamic "slab" upper ocean, a layer of water usually around $50 \mathrm{~m}$ thick that calculates only temperature (sometimes referred to as a "mixed-layer model"). Such air-sea coupling allows those models to include a seasonal cycle of solar radiation. The sea surface temperatures (SSTs) respond to increases in carbon dioxide $\left(\mathrm{CO}_{2}\right)$, but there is no ocean dynamical response to the changing climate. Since the full depth of the ocean is not included, computing requirements are relatively modest so these models can be run to equilibrium with a doubling of atmospheric $\mathrm{CO}_{2}$. This model design was prevalent through the 1980s, and results from such equilibrium simulations were an early basis of societal concern about the consequences of increasing $\mathrm{CO}_{2}$.

However, such equilibrium (steady-state) experiments provide no information on time-dependent climate change and no information on rates of climate change. In the late 1980s, more comprehensive fully coupled global ocean/atmosphere/seaice/land-surface climate models (also referred to as AtmosphereOcean Global Climate Models, Atmosphere-Ocean General Circulation Models or simply AOGCMs) began to be run with slowly increasing $\mathrm{CO}_{2}$, and preliminary results from two such models appeared in the 1990 IPCC Assessment (IPCC, 1990).

In the 1992 IPCC update prior to the Earth Summit in Rio de Janeiro (IPCC, 1992), there were results from four AOGCMs run with $\mathrm{CO}_{2}$ increasing at $1 \% / \mathrm{yr}$ to doubling around year 70 of the simulations (these were standardised sensitivity experiments, and consequently no actual dates were attached). Inclusion of the full ocean meant that warming at high latitudes was not as uniform as from the non-dynamic mixed-layer models. In regions of deep ocean mixing in the North Atlantic and Southern Oceans, warming was less than at other high latitude locations. Three of those four models used some form of flux adjustment whereby the fluxes of heat, fresh water and momentum were either singly or in some combination adjusted at the air-sea interface to account for incompatibilities in the component models. However, the assessment of those models suggested that the main results concerning the patterns and magnitudes of the climate changes in the model without flux adjustment were essentially the same as in the flux-adjusted models.

The most recent IPCC Second Assessment Report (IPCC, 1996) (hereafter SAR) included a much more extensive collection of global coupled climate model results from models run with what became a standard $1 \% / \mathrm{yr} \mathrm{CO}_{2}$-increase experiment. These models corroborated the results in the earlier assessment regarding the time evolution of warming and the reduced warming in regions of deep ocean mixing. There were additional studies of changes in variability in the models in addition to changes in the mean, and there were more results concerning possible changes in climate extremes. Information on possible future changes of regional climate was included as well.

The SAR also included results from the first two global coupled models run with a combination of increasing $\mathrm{CO}_{2}$ and sulphate aerosols for the 20th and 21st centuries. Thus, for the first time, models were run with a more realistic forcing history for the 20th century and allowed the direct comparison of the model's response to the observations. The combination of the warming effects on a global scale from increasing $\mathrm{CO}_{2}$ and the regional cooling from the direct effect of sulphate aerosols produced a better agreement with observations of the time evolution of the globally averaged warming and the patterns of 20th century climate change. Subsequent experiments have attempted to quantify and include additional forcings for 20th century climate (Chapter 8), with projected outcomes for those forcings in scenario integrations into the 21 st century discussed below.

In the SAR, the two global coupled model runs with the combination of $\mathrm{CO}_{2}$ and direct effect of sulphate aerosols both gave a warming at mid-21st century relative to 1990 of around $1.5^{\circ} \mathrm{C}$. To investigate more fully the range of forcing scenarios and uncertainty in climate sensitivity (defined as equilibrium globally averaged surface air temperature increase due to a doubling of $\mathrm{CO}_{2}$, see discussion in Section 9.2 below) a simpler climate model was used. Combining low emissions with low sensitivity and high emissions with high sensitivity gave an extreme range of 1 to $4.5^{\circ} \mathrm{C}$ for the warming in the simple model at the year 2100 (assuming aerosol concentrations constant at 1990-levels). These projections were generally lower than corresponding projections in IPCC (1990) because of the inclusion of aerosols in the pre-1990 radiative forcing history. When the possible effects of future changes of anthropogenic aerosol as prescribed in the IS92 scenarios were incorporated this led to lower projections of temperature change of between $1^{\circ} \mathrm{C}$ and $3.5^{\circ} \mathrm{C}$ with the simple model.

Spatial patterns of climate change simulated by the global coupled models in the SAR corroborated the IPCC (1990) results. With increasing greenhouse gases the land was projected to warm generally more than the oceans, with a maximum annual mean warming in high latitudes associated with reduced snow cover and increased runoff in winter, with greatest warming at 
high northern latitudes. Including the effects of aerosols led to a somewhat reduced warming in middle latitudes of the Northern Hemisphere and the maximum warming in northern high latitudes was less extensive since most sulphate aerosols are produced in the Northern Hemisphere. All models produced an increase in global mean precipitation but at that time there was little agreement among models on changes in storminess in a warmer world and conclusions regarding extreme storm events were even more uncertain.

\subsubsection{New Types of Model Experiments since 1995}

The progression of experiments including additional forcings has continued and new experiments with additional greenhouse gases (such as ozone, CFCs, etc., as well as $\mathrm{CO}_{2}$ ) will be assessed in this chapter.

In contrast to the two global coupled climate models in the 1990 Assessment, the Coupled Model Intercomparison Project (CMIP) (Meehl et al., 2000a) includes output from about twenty AOGCMs worldwide, with roughly half of them using flux adjustment. Nineteen of them have been used to perform idealised $1 \% / y r$ $\mathrm{CO}_{2}$-increase climate change experiments suitable for direct intercomparison and these are analysed here. Roughly half that number have also been used in more detailed scenario experiments with time evolutions of forcings including at least $\mathrm{CO}_{2}$ and sulphate aerosols for 20th and 21st century climate. Since there are some differences in the climate changes simulated by various models even if the same forcing scenario is used, the models are compared to assess the uncertainties in the responses. The comparison of 20th century climate simulations with observations (see Chapter 8) has given us more confidence in the abilities of the models to simulate possible future climate changes in the 21 st century and reduced the uncertainty in the model projections (see Chapter 14). The newer model integrations without flux adjustment give us indications of how far we have come in removing biases in the model components. The results from CMIP confirm what was noted in the SAR in that the basic patterns of climate system response to external forcing are relatively robust in models with and without flux adjustment (Gregory and Mitchell, 1997; Fanning and Weaver, 1997; Meehl et al., 2000a). This also gives us more confidence in the results from the models still using flux adjustment.

The IPCC data distribution centre (DDC) has collected results from a number of transient scenario experiments. They start at an early time of industrialisation and most have been run with and without the inclusion of the direct effect of sulphate aerosols. Note that most models do not use other forcings described in Chapter 6 such as soot, the indirect effect of sulphate aerosols, or land-use changes. Forcing estimates for the direct effect of sulphate aerosols and other trace gases included in the DDC models are given in Chapter 6. Several models also include effects of tropospheric and stratospheric ozone changes.

Additionally, multi-member ensemble integrations have been run with single models with the same forcing. So-called "stabilisation" experiments have also been run with the atmospheric greenhouse gas concentrations increasing by $1 \% / \mathrm{yr}$ or following an IPCC scenario, until $\mathrm{CO}_{2}$-doubling, tripling or quadrupling. The greenhouse gas concentration is then kept fixed and the model integrations continue for several hundred years in order to study the commitment to climate change. The $1 \% / y r$ rate of increase for future climate, although larger than actual $\mathrm{CO}_{2}$ increase observed to date, is meant to account for the radiative effects of $\mathrm{CO}_{2}$ and other trace gases in the future and is often referred to as "equivalent $\mathrm{CO}_{2}$ " (see discussion in Section 9.2.1). This rate of increase in radiative forcing is often used in model intercomparison studies to assess general features of model response to such forcing.

In 1996, the IPCC began the development of a new set of emissions scenarios, effectively to update and replace the wellknown IS92 scenarios. The approved new set of scenarios is described in the IPCC Special Report on Emission Scenarios (SRES) (Nakićenović et al., 2000; see more complete discussion of SRES scenarios and forcing in Chapters 3, 4, 5 and 6). Four different narrative storylines were developed to describe consistently the relationships between emission driving forces and their evolution and to add context for the scenario quantification (see Box 9.1). The resulting set of forty scenarios (thirty-five of which contain data on the full range of gases required for climate modelling) cover a wide range of the main demographic, economic and technological driving forces of future greenhouse gas and sulphur emissions. Each scenario represents a specific quantification of one of the four storylines. All the scenarios based on the same storyline constitute a scenario "family". (See Box 9.1, which briefly describes the main characteristics of the four SRES storylines and scenario families.) The SRES scenarios do not include additional climate initiatives, which means that no scenarios are included that explicitly assume implementation of the UNFCCC or the emissions targets of the Kyoto Protocol. However, greenhouse gas emissions are directly affected by non-climate change policies designed for a wide range of other purposes. Furthermore, government policies can, to varying degrees, influence the greenhouse gas emission drivers and this influence is broadly reflected in the storylines and resulting scenarios.

Because SRES was not approved until 15 March 2000, it was too late for the modelling community to incorporate the scenarios into their models and have the results available in time for this Third Assessment Report. Therefore, in accordance with a decision of the IPCC Bureau in 1998 to release draft scenarios to climate modellers (for their input to the Third Assessment Report) one marker scenario was chosen from each of four of the scenario groups based on the storylines (A1B, A2, B1 and B2) (Box 9.1). The choice of the markers was based on which initial quantification best reflected the storyline, and features of specific models. Marker scenarios are no more or less likely than any other scenarios but these scenarios have received the closest scrutiny. Scenarios were also selected later to illustrate the other two scenario groups (A1FI and A1T), hence there is an illustrative scenario for each of the six scenario groups. These latter two illustrative scenarios were not selected in time for AOGCM models to utilise them in this report. In fact, time and computer resource limitations dictated that most modelling groups could run only A2 and B2, and results from those integrations are evaluated in this chapter. However, results for all six illustrative scenarios are shown here using a simple climate model discussed below. The IS92a scenario is also used in a number of the results presented in this chapter in order to provide direct comparison with the results in the SAR. 
Box 9.1: The Emissions Scenarios of the Special Report on Emissions Scenarios (SRES)

A1. The A1 storyline and scenario family describe a future world of very rapid economic growth, global population that peaks in mid-century and declines thereafter, and the rapid introduction of new and more efficient technologies. Major underlying themes are convergence among regions, capacity building and increased cultural and social interactions, with a substantial reduction in regional differences in per capita income. The A1 scenario family develops into three groups that describe alternative directions of technological change in the energy system. The three A1 groups are distinguished by their technological emphasis: fossil intensive (A1FI), non-fossil energy sources (A1T), or a balance across all sources (A1B) (where balanced is defined as not relying too heavily on one particular energy source, on the assumption that similar improvement rates apply to all energy supply and end use technologies).

A2. The A2 storyline and scenario family describe a very heterogeneous world. The underlying theme is self-reliance and preservation of local identities. Fertility patterns across regions converge very slowly, which results in continuously increasing population. Economic development is primarily regionally oriented and per capita economic growth and technological change are more fragmented and slower than in other storylines.

B1. The B1 storyline and scenario family describe a convergent world with the same global population, that peaks in mid-century and declines thereafter, as in the A1 storyline, but with rapid change in economic structures toward a service and information economy, with reductions in material intensity and the introduction of clean and resource-efficient technologies. The emphasis is on global solutions to economic, social and environmental sustainability, including improved equity, but without additional climate initiatives.

B2. The B2 storyline and scenario family describe a world in which the emphasis is on local solutions to economic, social and environmental sustainability. It is a world with continuously increasing global population, at a rate lower than A2, intermediate levels of economic development, and less rapid and more diverse technological change than in the B1 and A1 storylines. While the scenario is also oriented towards environmental protection and social equity, it focuses on local and regional levels.

The final four marker scenarios contained in SRES differ in minor ways from the draft scenarios used for the AOGCM experiments described in this report. In order to ascertain the likely effect of differences in the draft and final SRES scenarios each of the four draft and final marker scenarios were studied using a simple climate model tuned to the AOGCMs used in this report. For three of the four marker scenarios (A1B, A2 and B2) temperature change from the draft and final scenarios are very similar. The primary difference is a change to the standardised values for 1990 to 2000, which is common to all these scenarios. This results in a higher forcing early in the period. There are further small differences in net forcing, but these decrease until, by 2100 , differences in temperature change in the two versions of these scenarios are in the range 1 to $2 \%$. For the B1 scenario, however, temperature change is significantly lower in the final version, leading to a difference in the temperature change in 2100 of almost $20 \%$, as a result of generally lower emissions across the full range of greenhouse gases. For descriptions of the simulations, see Section 9.3.1.

\subsection{Climate and Climate Change}

Chapter 1 discusses the nature of the climate system and the climate variability and change it may undergo, both naturally and as a consequence of human activity. The projections of future climate change discussed in this chapter are obtained using climate models in which changes in atmospheric composition are specified. The models "translate" these changes in composition into changes in climate based on the physical processes governing the climate system as represented in the models. The simulated climate change depends, therefore, on projected changes in emissions, the changes in atmospheric greenhouse gas and particulate (aerosol) concentrations that result, and the manner in which the models respond to these changes. The response of the climate system to a given change in forcing is broadly characterised by its "climate sensitivity". Since the climate system requires many years to come into equilibrium with a change in forcing, there remains a "commitment" to further climate change even if the forcing itself ceases to change.

Observations of the climate system and the output of models are a combination of a forced climate change "signal" and internally generated natural variability which, because it is random and unpredictable on long climate time-scales, is characterised as climate "noise". The availability of multiple simulations from a given model with the same forcing, and of simulations from many models with similar forcing, allows ensemble methods to be used to better characterise projected climate change and the agreement or disagreement (a measure of reliability) of model results.

\subsubsection{Climate Forcing and Climate Response}

\section{The heat balance}

Broad aspects of global mean temperature change may be illustrated using a simple representation of the heat budget of the climate system expressed as:

$$
\mathrm{dH} / \mathrm{dt}=\mathrm{F}-\alpha \mathrm{T} \text {. }
$$


Here $\mathrm{F}$ is the radiative forcing change as discussed in Chapter 6; $\alpha \mathrm{T}$ represents the net effect of processes acting to counteract changes in mean surface temperature, and $\mathrm{dH} / \mathrm{dt}$ is the rate of heat storage in the system. All terms are differences from unperturbed equilibrium climate values. A positive forcing will act to increase the surface temperature and the magnitude of the resulting increase will depend on the strength of the feedbacks measured by $\alpha \mathrm{T}$. If $\alpha$ is large, the temperature change needed to balance a given change in forcing is small and vice versa. The result will also depend on the rate of heat storage which is dominated by the ocean so that $\mathrm{dH} / \mathrm{dt}=\mathrm{dH}_{\mathrm{o}} / \mathrm{dt}=\mathrm{F}_{\mathrm{o}}$ where $\mathrm{H}_{\mathrm{o}}$ is the ocean heat content and $\mathrm{F}_{\mathrm{o}}$ is the flux of heat into the ocean. With this approximation the heat budget becomes $\mathrm{F}=\alpha \mathrm{T}+\mathrm{F}_{\mathrm{o}}$, indicating that both the feedback term and the flux into the ocean act to balance the radiative forcing for non-equilibrium conditions.

\section{Radiative forcing in climate models}

A radiative forcing change, symbolised by $\mathrm{F}$ above, can result from changes in greenhouse gas concentrations and aerosol loading in the atmosphere. The calculation of $\mathrm{F}$ is discussed in Chapter 6 where a new estimate of $\mathrm{CO}_{2}$ radiative forcing is given which is smaller than the value in the SAR. According to Section 6.3.1, the lower value is due mainly to the fact that stratospheric temperature adjustment was not included in the (previous) estimates given for the forcing change. It is important to note that this new radiative forcing estimate does not affect the climate change and equilibrium climate sensitivity calculations made with general circulation models. The effect of a change in greenhouse gas concentration and/or aerosol loading in a general circulation model (GCM) is calculated internally and interactively based on, and in turn affecting, the three dimensional state of the atmosphere. In particular, the stratospheric temperature responds to changes in radiative fluxes due to changes in $\mathrm{CO}_{2}$ concentration and the GCM calculation includes this effect.

\section{Equivalent $\mathrm{CO}_{2}$}

The radiative effects of the major greenhouse gases which are well-mixed throughout the atmosphere are often represented in GCMs by an "equivalent" $\mathrm{CO}_{2}$ concentration, namely the $\mathrm{CO}_{2}$ concentration that gives a radiative forcing equal to the sum of the forcings for the individual greenhouse gases. When used in simulations of forced climate change, the increase in "equivalent $\mathrm{CO}_{2}$ " will be larger than that of $\mathrm{CO}_{2}$ by itself, since it also accounts for the radiative effects of other gases.

\section{$1 \% / y r$ increasing $\mathrm{CO}_{2}$}

A common standardised forcing scenario specifies atmospheric $\mathrm{CO}_{2}$ to increase at a rate of $1 \% / y e a r$ compound until the concentration doubles (or quadruples) and is then held constant. The $\mathrm{CO}_{2}$ content of the atmosphere has not, and likely will not, increase at this rate (let alone suddenly remain constant at twice or four times an initial value). If regarded as a proxy for all greenhouse gases, however, an "equivalent $\mathrm{CO}_{2}$ " increase of $1 \% / \mathrm{yr}$ does give a forcing within the range of the SRES scenarios.

This forcing prescription is used to illustrate and to quantify aspects of AOGCM behaviour and provides the basis for the analysis and intercomparison of modelled responses to a specified forcing change (e.g., in the SAR and the CMIP2 intercomparison). The resulting information is also used to calibrate simpler models which may then be employed to investigate a broad range of forcing scenarios as is done in Section 9.3.3. Figure 9.1 illustrates the global mean temperature evolution for this standardised forcing in a simple illustrative example with no exchange with the deep ocean (the green curves) and for a full coupled AOGCM (the red curves). The diagram also illustrates the transient climate response, climate sensitivity and warming commitment.

\section{$T C R$ - Transient climate response}

The temperature change at any time during a climate change integration depends on the competing effects of all of the processes that affect energy input, output, and storage in the ocean. In particular, the global mean temperature change which occurs at the time of $\mathrm{CO}_{2}$ doubling for the specific case of a $1 \% / \mathrm{yr}$ increase of $\mathrm{CO}_{2}$ is termed the "transient climate response" (TCR) of the system. This temperature change, indicated in Figure 9.1, integrates all processes operating in the system, including the strength of the feedbacks and the rate of heat storage in the ocean, to give a straightforward measure of model response to a change in forcing. The range of TCR values serves to illustrate and calibrate differences in model response to the same standardised forcing. Analogous TCR measures may be used, and compared among models, for other forcing scenarios.

\section{Equilibrium climate sensitivity}

The "equilibrium climate sensitivity" (IPCC 1990, 1996) is defined as the change in global mean temperature, $\mathrm{T}_{2 \mathrm{x}}$, that results when the climate system, or a climate model, attains a new equilibrium with the forcing change $\mathrm{F}_{2 \mathrm{x}}$ resulting from a doubling of the atmospheric $\mathrm{CO}_{2}$ concentration. For this new equilibrium $\mathrm{dH} / \mathrm{dt}=0$ in the simple heat budget equation and $\mathrm{F}_{2 \mathrm{x}}=\alpha \mathrm{T}_{2 \mathrm{x}}$ indicating a balance between energy input and output. The equilibrium climate sensitivity

$$
\mathrm{T}_{2 \mathrm{x}}=\mathrm{F}_{2 \mathrm{x}} / \alpha
$$

is inversely proportional to $\alpha$, which measures the strength of the feedback processes in the system that act to counter a change in forcing. The equilibrium climate sensitivity is a straightforward, although averaged, measure of how the system responds to a specific forcing change and may be used to compare model responses, calibrate simple climate models, and to scale temperature changes in other circumstances.

In earlier assessments, the climate sensitivity was obtained from calculations made with AGCMs coupled to mixed-layer upper ocean models (referred to as mixed-layer models). In that case there is no exchange of heat with the deep ocean and a model can be integrated to a new equilibrium in a few tens of years. For a full coupled atmosphere/ocean GCM, however, the heat exchange with the deep ocean delays equilibration and several millennia, rather than several decades, are required to attain it. This difference is illustrated in Figure 9.1 where the smooth green curve illustrates the rapid approach to a new climate equilibrium in an idealised mixed-layer case while the red curve is the result of a coupled model integration and indicates the much longer time needed to attain equilibrium when there is interaction with the deep ocean. 


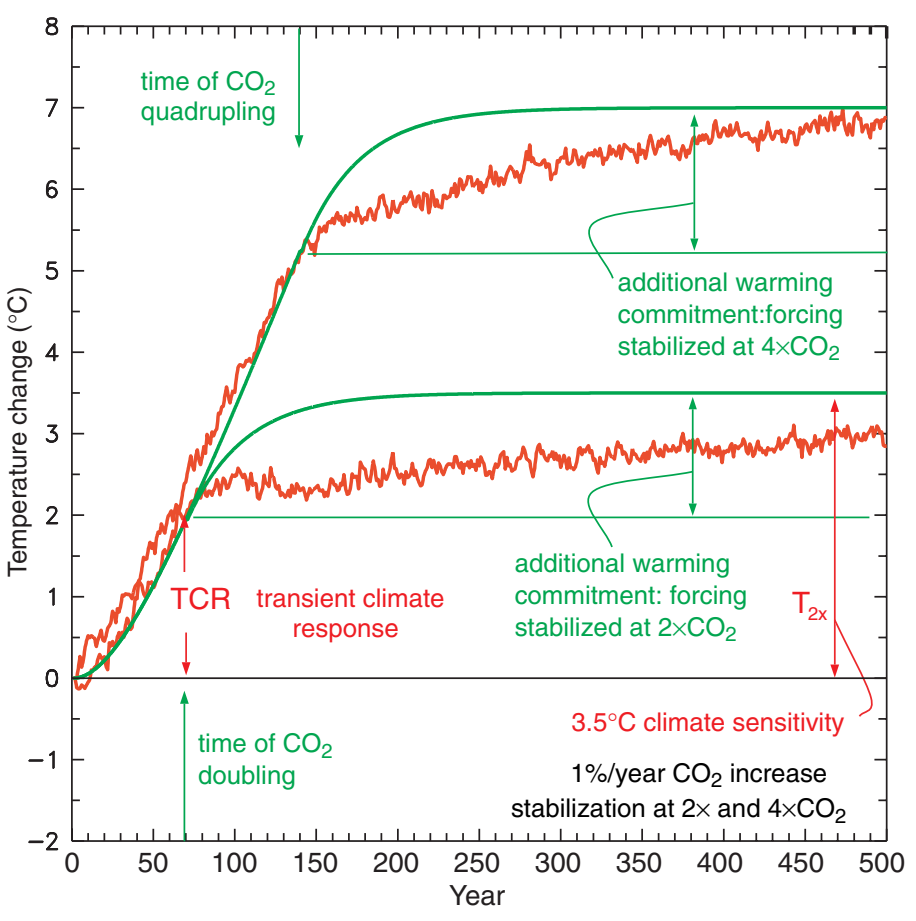

Figure 9.1: Global mean temperature change for $1 \% / \mathrm{yr} \mathrm{CO}_{2}$ increase with subsequent stabilisation at $2 \times \mathrm{CO}_{2}$ and $4 \times \mathrm{CO}_{2}$. The red curves are from a coupled AOGCM simulation (GFDL_R15_a) while the green curves are from a simple illustrative model with no exchange of energy with the deep ocean. The "transient climate response", TCR, is the temperature change at the time of $\mathrm{CO}_{2}$ doubling and the "equilibrium climate sensitivity", $\mathrm{T}_{2 \mathrm{x}}$, is the temperature change after the system has reached a new equilibrium for doubled $\mathrm{CO}_{2}$, i.e., after the "additional warming commitment" has been realised.

\section{Effective climate sensitivity}

Although the definition of equilibrium climate sensitivity is straightforward, it applies to the special case of equilibrium climate change for doubled $\mathrm{CO}_{2}$ and requires very long simulations to evaluate with a coupled model. The "effective climate sensitivity" is a related measure that circumvents this requirement. The inverse of the feedback term $\alpha$ is evaluated from model output for evolving non-equilibrium conditions as

$$
1 / \alpha_{\mathrm{e}}=\mathrm{T} /\left(\mathrm{F}-\mathrm{dH}_{\mathrm{o}} / \mathrm{dt}\right)=\mathrm{T} /\left(\mathrm{F}-\mathrm{F}_{\mathrm{o}}\right)
$$

and the effective climate sensitivity is calculated as

$$
\mathrm{T}_{\mathrm{e}}=\mathrm{F}_{2 \mathrm{x}} / \alpha_{\mathrm{e}}
$$

with units and magnitudes directly comparable to the equilibrium sensitivity. The effective sensitivity becomes the equilibrium sensitivity under equilibrium conditions with $2 \times \mathrm{CO}_{2}$ forcing. The effective climate sensitivity is a measure of the strength of the feedbacks at a particular time and it may vary with forcing history and climate state.

\section{Warming commitment}

An increase in forcing implies a "commitment" to future warming even if the forcing stops increasing and is held at a constant value. At any time, the "additional warming commitment" is the further increase in temperature, over and above the increase that has already been experienced, that will occur before the system reaches a new equilibrium with radiative forcing stabilised at the current value. This behaviour is illustrated in Figure 9.1 for the idealised case of instantaneous stabilisation at $2 \times$ and $4 \times \mathrm{CO}_{2}$. Analogous behaviour would be seen for more realistic stabilisation scenarios.

\subsubsection{Simulating Forced Climate Change}

\subsubsection{Signal versus noise}

A climate change simulation produces a time evolving three dimensional distribution of temperature and other climate variables. For the real system or for a model, and taking temperature as an example, this is expressed as $\mathrm{T}=\mathrm{T}_{0}+\mathrm{T}_{0}$ ' for preindustrial equilibrium conditions. $\mathrm{T}$ is now the full temperature field rather than the global mean temperature change of Section 9.2.1. $\mathrm{T}_{0}$ represents the temperature structure of the mean climate, which is determined by the (pre-industrial) forcing, and $\mathrm{T}_{0}{ }^{\prime}$ the internally generated random natural variability with zero mean. For climate which is changing as a consequence of increasing atmospheric greenhouse gas concentrations or other forcing changes, $T=T_{0}+T_{f}+T^{\prime}$ where $T_{f}$ is the deterministic climate change caused by the changing forcing, and $\mathrm{T}^{\prime}$ is the natural variability under these changing conditions. Changes in the statistics of the natural variability, that is in the statistics of $\mathrm{T}_{0}{ }^{\prime}$ vs $\mathrm{T}^{\prime}$, are of considerable interest and are discussed in Sections 9.3.5 and 9.3.6 which treat changes in variability and extremes.

The difference in temperature between the control and climate change simulations is written as $\Delta \mathrm{T}=\mathrm{T}_{\mathrm{f}}+\left(\mathrm{T}^{\prime}-\mathrm{T}_{0}\right)=\mathrm{T}_{\mathrm{f}}$ $+T^{\prime \prime}$, and is a combination of the deterministic signal $T_{f}$ and a random component $\mathrm{T}^{\prime \prime}=\mathrm{T}^{\prime}-\mathrm{T}_{0}{ }^{\prime}$ which has contributions from the natural variability of both simulations. A similar expression arises when calculating climate change as the difference between an earlier and a later period in the observations or a simulation. Observed and simulated climate change are the sum of the forced "signal" and the natural variability "noise" and it is important to be able to separate the two. The natural variability that obscures the forced signal may be at least partially reduced by averaging.

\subsubsection{Ensembles and averaging}

An ensemble consists of a number of simulations undertaken with the same forcing scenario, so that the forced change $T_{f}$ is the same for each, but where small perturbations to remote initial conditions result in internally generated climate variability that is different for each ensemble member. Small ensembles of simulations have been performed with a number of models as indicated in the "number of simulations" column in Table 9.1. Averaging over the ensemble of results, indicated by braces, gives the ensemble mean climate change as $\{\Delta \mathrm{T}\}=$ $\mathrm{T}_{\mathrm{f}}+\left\{\mathrm{T}^{\prime \prime}\right\}$. For independent realisations, the natural variability noise is reduced by the ensemble averaging (averaging to zero for a large enough ensemble) so that $\{\Delta \mathrm{T}\}$ is an improved estimate of the model's forced climate change $T_{\mathrm{f}}$. This is illustrated in Figure 9.2, which shows the simulated temperature differences from 1975 to 1995 to the first decade in the 21st century for three climate change simulations made with the same model and the same forcing scenario but starting from 

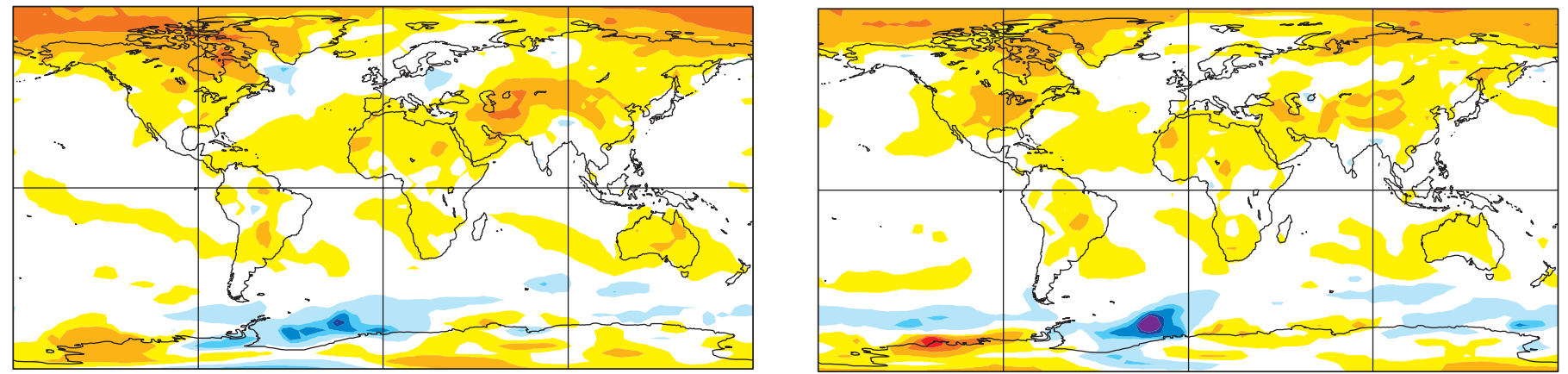

1

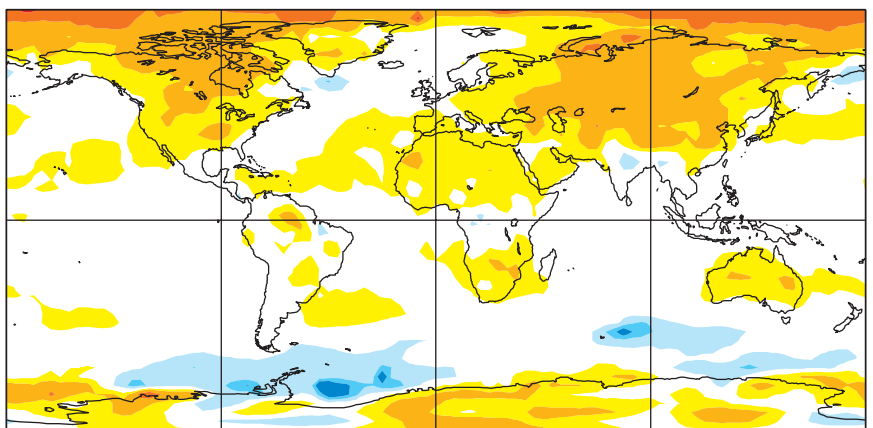

2

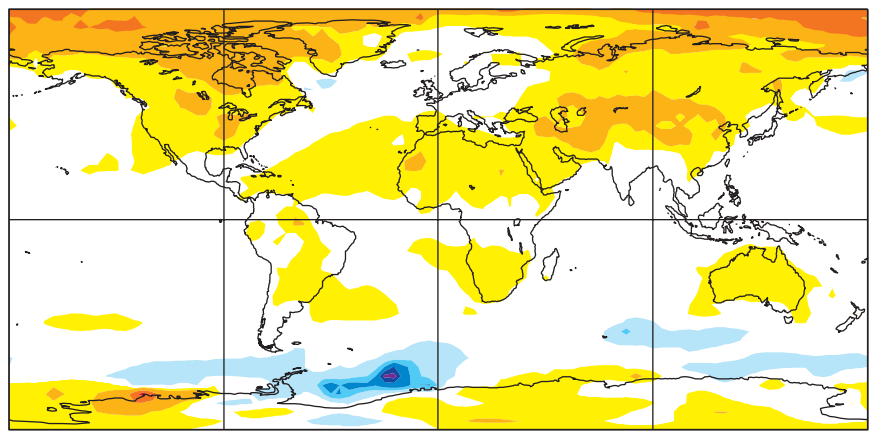

3

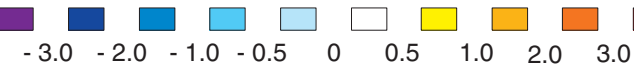

ENSEMBLE MEAN

Figure 9.2: Three realisations of the geographical distribution of temperature differences from 1975 to 1995 to the first decade in the 21 st century made with the same model (CCCma CGCM1) and the same IS92a greenhouse gas and aerosol forcing but with slightly different initial conditions a century earlier. The ensemble mean is the average of the three realisations. (Unit: ${ }^{\circ} \mathrm{C}$ ).

slightly different initial conditions more than a century earlier. The differences between the simulations reflect differences in the natural variability. The ensemble average over the three realisations, also shown in the diagram, is an estimate of the model's forced climate change where some of this natural variability has been averaged out.

The ensemble variance for a particular model, assuming there is no correlation between the forced component and the variability, is $\sigma_{\Delta \mathrm{T}}^{2}=\left\{(\Delta \mathrm{T}-\{\Delta \mathrm{T}\})^{2}\right\}=\left\{\left(\mathrm{T}^{\prime \prime}-\left\{\mathrm{T}^{\prime \prime}\right\}\right)^{2}\right\}=\sigma_{\mathrm{N}}^{2}$ which gives a measure of the natural variability noise. The "signal to noise ratio", $\{\Delta \mathrm{T}\} / \sigma_{\Delta \mathrm{T}}$, compares the strength of the climate change signal to this natural variability noise. The signal stands out against the noise when and where this ratio is large. The signal will be better represented by the ensemble mean as the size of the ensemble grows and the noise is averaged out over more independent realisations. This is indicated by the width, $\{\Delta \mathrm{T}\} \pm 2 \sigma_{\Delta \mathrm{T}} / \mathrm{N} \mathrm{n}$, of the approximate $95 \%$ confidence interval which decreases as the ensemble size $\mathrm{n}$ increases.

The natural variability may be further reduced by averaging over more realisations, over longer time intervals, and by averaging in space, although averaging also affects the information content of the result. In what follows, the geographical distributions $\Delta \mathrm{T}$, zonal averages $[\Delta \mathrm{T}]$, and global averages $<\Delta \mathrm{T}>$ of temperature and other variables are discussed. As the amount of averaging increases, the climate change signal is better defined, since the noise is increasingly averaged out, but the geographical information content is reduced.

\subsubsection{Multi-model ensembles}

The collection of coupled climate model results that is available for this report permits a multi-model ensemble approach to the synthesis of projected climate change. Multi-model ensemble approaches are already used in short-range climate forecasting (e.g., Graham et al., 1999; Krishnamurti et al., 1999; Brankovic and Palmer, 2000; Doblas-Reyes et al., 2000; Derome et al., 2001). When applied to climate change, each model in the ensemble produces a somewhat different projection and, if these represent plausible solutions to the governing equations, they may be considered as different realisations of the climate change drawn from the set of models in active use and produced with current climate knowledge. In this case, temperature is represented as $\mathrm{T}=\mathrm{T}_{0}+\mathrm{T}_{\mathrm{F}}+\mathrm{T}_{\mathrm{m}}+\mathrm{T}^{\prime}$ where $\mathrm{T}_{\mathrm{F}}$ is the deterministic forced climate change for the real system and $T_{m}=T_{f}-T_{F}$ is the error in the model's simulation of this forced response. $T^{\prime}$ now also includes errors in the statistical behaviour of the simulated natural variability. The multi-model ensemble mean estimate of forced climate change is $\{\Delta \mathrm{T}\}=\mathrm{T}_{\mathrm{F}}+\left\{\mathrm{T}_{\mathrm{m}}\right\}+\left\{\mathrm{T}^{\prime \prime}\right\}$ where the natural variability again averages to zero for a large enough ensemble. To the extent that unrelated model errors tend to average out, the ensemble mean or systematic error $\left\{\mathrm{T}_{\mathrm{m}}\right\}$ will be small, $\{\Delta \mathrm{T}\}$ will approach $\mathrm{T}_{\mathrm{F}}$ and the multi-model ensemble average will be a better estimate of the forced climate change of the real system than the result from a particular model.

As noted in Chapter 8, no one model can be chosen as "best" and it is important to use results from a range of models. Lambert 
and Boer (2001) show that for the CMIP1 ensemble of simulations of current climate, the multi-model ensemble means of temperature, pressure, and precipitation are generally closer to the observed distributions, as measured by mean squared differences, correlations, and variance ratios, than are the results of any particular model. The multi-model ensemble mean represents those features of projected climate change that survive ensemble averaging and so are common to models as a group. The multimodel ensemble variance, assuming no correlation between the forced and variability components, is $\sigma^{2}{ }_{\Delta \mathrm{T}}=\sigma^{2}{ }_{\mathrm{M}}+\sigma^{2}{ }_{\mathrm{N}}$, where $\sigma^{2}{ }_{M}=\left\{\left(T_{m}-\left\{T_{m}\right\}\right)^{2}\right\}$ measures the inter-model scatter of the forced component and $\sigma^{2}$ the natural variability. The common signal is again best discerned where the signal to noise ratio $\{\Delta \mathrm{T}\}$ $/ \sigma_{\Delta \mathrm{T}}$ is largest.

Figure 9.3 illustrates some basic aspects of the multi-model ensemble approach for global mean temperature and precipitation. Each model result is the sum of a smooth forced signal, $\mathrm{T}_{\mathrm{f}}$ and the accompanying natural variability noise. The natural variability is different for each model and tends to average out so that the ensemble mean estimates the smooth forced signal. The scatter of results about the ensemble mean (measured by the ensemble variance) is an indication of uncertainty in the results and is seen to increase with time. Global mean temperature is seen to be a more robust climate change variable than precipitation in the sense that $\{\Delta \mathrm{T}\} / \sigma_{\Delta \mathrm{T}}$ is larger than $\{\Delta \mathrm{P}\} / \sigma_{\Delta \mathrm{P}}$. These results are discussed further in Section 9.3.2.

\subsubsection{Uncertainty}

Projections of climate change are affected by a range of uncertainties (see also Chapter 14) and there is a need to discuss and to quantify uncertainty in so far as is possible. Uncertainty in projected climate change arises from three main sources; uncertainty in forcing scenarios, uncertainty in modelled responses to given forcing scenarios, and uncertainty due to missing or misrepresented physical processes in models. These are discussed in turn below.

Forcing scenarios: The use of a range of forcing scenarios reflects uncertainties in future emissions and in the resulting greenhouse gas concentrations and aerosol loadings in the atmosphere. The complexity and cost of full AOGCM simulations has restricted these calculations to a subset of scenarios; these are listed in Table 9.1 and discussed in Section 9.3.1. Climate projections for the remaining scenarios are made with less general models and this introduces a further level of uncertainty. Section 9.3.2 discusses global mean warming for a broad range of scenarios obtained with simple models calibrated with AOGCMs. Chapter 13 discusses a number of techniques for scaling AOGCM results from a particular forcing scenario to apply to other scenarios.

Model response: The ensemble standard deviation and the range are used as available indications of uncertainty in model results for a given forcing, although they are by no means a complete characterisation of the uncertainty. There are a number of caveats associated with the ensemble approach. Common or systematic errors in the simulation of current climate (e.g., Gates et al., 1999; Lambert and Boer, 2001; Chapter 8) survive ensemble averaging and contribute error to the ensemble mean while not contributing to the standard deviation. A tendency for models to under-simulate the level of natural variability would result in an underestimate of ensemble variance. There is also the possibility of seriously flawed outliers in the ensemble corrupting the results. The ensemble approach nevertheless represents one of the few methods currently available for deriving information from the array of model results and it is used in this chapter to characterise projections of future climate.

Missing or misrepresented physics: No attempt has been made to quantify the uncertainty in model projections of climate change due to missing or misrepresented physics. Current models attempt to include the dominant physical processes that govern the behaviour and the response of the climate system to specified forcing scenarios. Studies of "missing" processes are often carried out, for instance of the effect of aerosols on cloud lifetimes, but until the results are well-founded, of appreciable magnitude, and robust in a range of models, they are considered to be studies of sensitivity rather than projections of climate change. Physical processes which are misrepresented in one or more, but not all, models will give rise to differences which will be reflected in the ensemble standard deviation.

The impact of uncertainty due to missing or misrepresented processes can, however, be limited by requiring model simulations to reproduce recent observed climate change. To the extent that errors are linear (i.e., they have proportionally the same impact on the past and future changes), it is argued in Chapter 12, Section 12.4.3.3 that the observed record provides a constraint on forecast anthropogenic warming rates over the coming decades that does not depend on any specific model's climate sensitivity, rate of ocean heat uptake and (under some scenarios) magnitude of sulphate forcing and response.

\subsection{Projections of Climate Change}

\subsubsection{Global Mean Response}

Since the SAR, there have been a number of new AOGCM climate simulations with various forcings that can provide estimates of possible future climate change as discussed in Section 9.1.2. For the first time we now have a reasonable number of climate simulations with different forcings so we can begin to quantify a mean climate response along with a range of possible outcomes. Here each model's simulation of a future climate state is treated as a possible outcome for future climate as discussed in the previous section.

These simulations fall into three categories (Table 9.1):

- The first are integrations with idealised forcing, namely, a $1 \% / \mathrm{yr}$ compound increase of $\mathrm{CO}_{2}$. This $1 \%$ increase represents equivalent $\mathrm{CO}_{2}$, which includes other greenhouse gases like methane, $\mathrm{NO}_{\mathrm{x}}$ etc. as discussed in Section 9.2.1. These runs extend at least to the time of effective $\mathrm{CO}_{2}$ doubling at year 70, and are useful for direct model intercomparisons since they use exactly the same forcing and thus are valuable to calibrate model response. These experiments are collected in the CMIP exercise (Meehl et al., 2000a) and referred to as "CMIP2" (Table 9.1). 
- A second category of AOGCM climate model simulations uses specified time-evolving future forcing where the simulations start sometime in the 19th century, and are run with estimates of observed forcing through the 20th century (see Chapter 8). That state is subsequently used to begin simulations of the future climate with estimated forcings of greenhouse gases ("G") or with the additional contribution from the direct effect of sulphate aerosols ("GS") according to various scenarios, such as IS92a (see Chapter 1). These simulations avoid the cold start problem (see SAR) present in the CMIP experiments. They allow evaluation of the model climate and response to forcing changes that could be experienced over the 21 st century. The experiments are collected in the IPCC-DDC. These experiments are assessed for the mid21 st century when most of the DDC experiments with sulphate aerosols finished.

- A third category are AOGCM simulations using as an initial state the end of the 20th century integrations, and then following the A2 and B2 (denoted as such in Table 9.1) draft marker SRES forcing scenarios to the year 2100 (see Section 9.1.2). These simulations are assessed to quantify possible future climate change at the end of the 21st century, and also are treated as members of an ensemble to better assess and quantify consistent climate changes. A simple model is also used to provide estimates of global temperature change for the end of the 21st century from a greater number of the SRES forcing scenarios.

Table 9.1 gives a detailed overview of all experiments assessed in this report.

\subsubsection{1\%/yr $\mathrm{CO}_{2}$ increase (CMIP2) experiments}

Figure 9.3 shows the global average temperature and precipitation changes for the nineteen CMIP2 simulations. At the time of $\mathrm{CO}_{2}$ doubling at year 70 , the 20 -year average (years 61 to 80 ) global mean temperature change (the transient climate response TCR; see Section 9.2) for these models is 1.1 to $3.1^{\circ} \mathrm{C}$ with an average of $1.8^{\circ} \mathrm{C}$ and a standard deviation of $0.4^{\circ} \mathrm{C}$ (Figure 9.7). This is similar to the SAR results (Figure 6.4 in Kattenberg et al., 1996).

At the time of $\mathrm{CO}_{2}$ doubling at year 70 , the 20 -year average (years 61 to 80) percentage change of the global mean precipitation for these models ranges from -0.2 to $5.6 \%$ with an average of $2.5 \%$ and a standard deviation of $1.5 \%$. This is similar to the SAR results.

For a hypothetical, infinite ensemble of experiments, in which $\mathrm{T}_{\mathrm{m}}$ and $\mathrm{T}^{\prime \prime}$ are uncorrelated and both have zero means,

$$
\left\{\Delta \mathrm{T}^{2}\right\}=\mathrm{T}_{\mathrm{f}}^{2}+\left\{\mathrm{T}_{\mathrm{m}}{ }^{2}\right\}+\left\{\mathrm{T}^{\prime \prime 2}\right\}=\mathrm{T}_{\mathrm{f}}^{2}+\sigma^{2}{ }_{\mathrm{M}}+\sigma^{2}{ }_{\mathrm{N}} .
$$

The ensemble mean square climate change is thus the sum of contributions from the common forced component $\left(\mathrm{T}_{\mathrm{f}}^{2}\right)$, model differences $\left(\sigma^{2}{ }_{M}\right)$, and internal variability $\left(\sigma^{2}{ }_{N}\right)$. This framework is applied to the CMIP2 experiments in Figure 9.4. These components of the total change are estimated for each grid box separately, using formulas that allow for unbiased estimates of these when a limited number of experiments are available (Räisänen 2000, 2001). The variance associated with internal
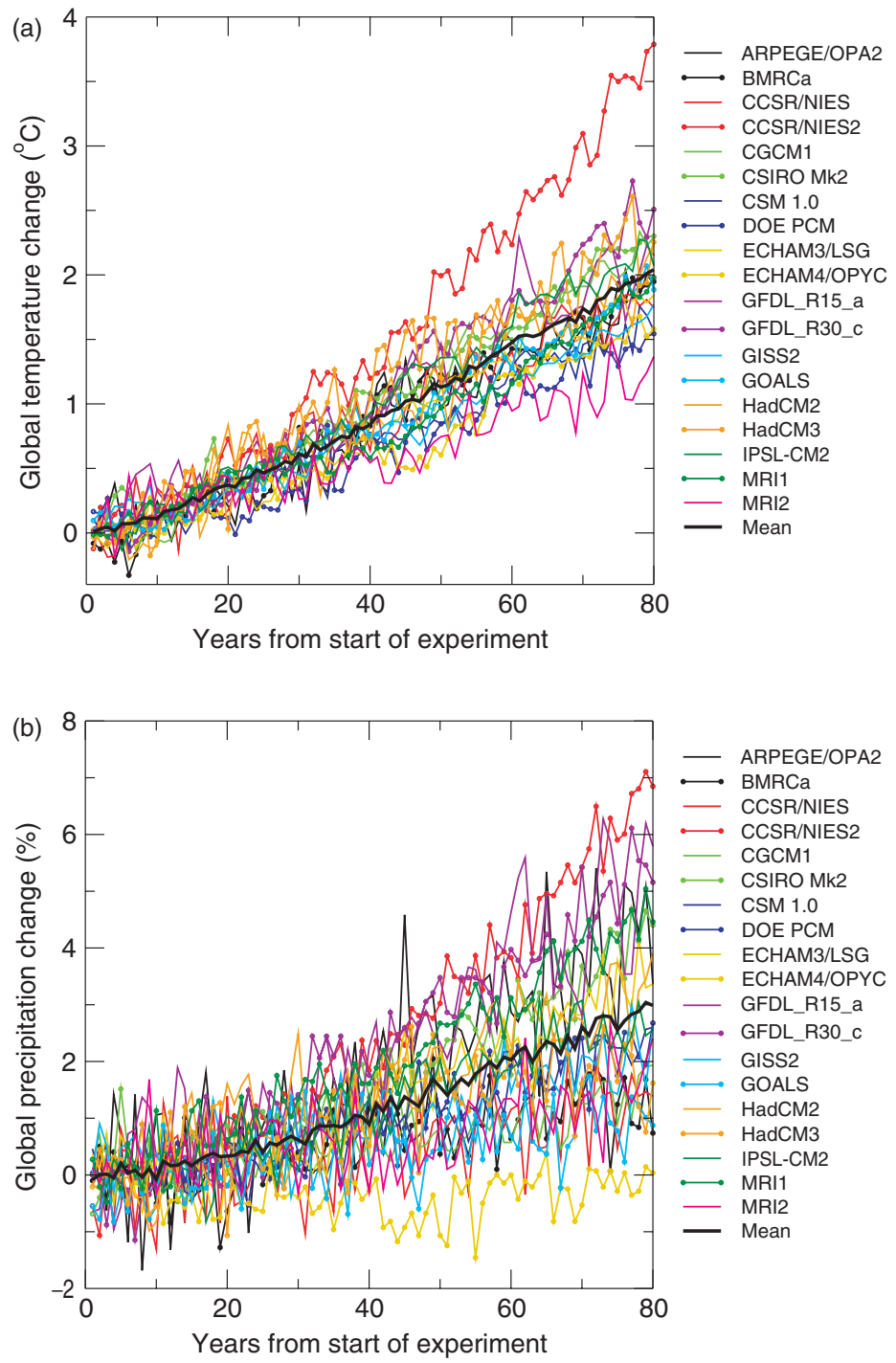

Figure 9.3: The time evolution of the globally averaged (a) temperature change relative to the control run of the CMIP2 simulations (Unit: ${ }^{\circ} \mathrm{C}$ ). (b) ditto. for precipitation. (Unit: \%). See Table 9.1 for more information on the individual models used here.

variability $\sigma^{2}{ }_{N}$ is inferred from the temporal variability of detrended $\mathrm{CO}_{2}$ run minus control run differences and the modelrelated variance $\sigma^{2}{ }_{\mathrm{M}}$ as a residual. Averaging the local statistics over the world, the relative agreement between the CMIP2 experiments is much higher for annual mean temperature changes (common signal makes up $86 \%$ of the total squared amplitude) than for precipitation (24\%) (Figure 9.4).

The relative agreement on seasonal climate changes is slightly lower, even though the absolute magnitude of the common signal is in some cases larger in the individual seasons than in the annual mean. Only 10 to $20 \%$ of the inter-experiment variance in temperature changes is attributable to internal variability, which indicates that most of this variance arises from differences between the models themselves. The estimated contribution of internal variability to the inter-experiment variance in precipitation changes is larger, from about a third in 
Table 9.1: The climate change experiments assessed in this report.

\begin{tabular}{|c|c|c|c|c|c|c|c|c|c|c|}
\hline $\begin{array}{c}\text { Model } \\
\text { Number } \\
(\text { see } \\
\text { Chapter 8, } \\
\text { Table 8.1) }\end{array}$ & $\begin{array}{l}\text { Model Name and } \\
\text { centre in italics } \\
\text { (see Chapter } 8 \text {, } \\
\text { Table 8.1) }\end{array}$ & Scenario name & Scenario description & $\begin{array}{l}\text { Number of } \\
\text { simulations }\end{array}$ & $\begin{array}{l}\text { Length of } \\
\text { simulation or } \\
\text { starting and } \\
\text { final year }\end{array}$ & $\begin{array}{c}\text { Transient } \\
\text { Climate } \\
\text { Response } \\
\text { (TCR) } \\
\text { (Section } \\
9.2 .1)\end{array}$ & 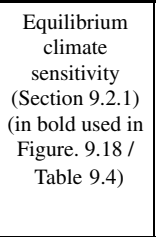 & $\begin{array}{c}\text { Effective } \\
\text { climate } \\
\text { sensitivity } \\
\text { (Section } \\
9.2 .1 \text { ) (from } \\
\text { CMIP2 yrs } \\
\text { 61-80) in bold } \\
\text { used in } \\
\text { Table A1 }\end{array}$ & References & Remarks \\
\hline 2 & $\begin{array}{l}\text { ARPEGE/OPA2 } \\
\text { CERFACS }\end{array}$ & CMIP2 & $1 \% \mathrm{CO}_{2}$ & 1 & 80 & 1.64 & & & $\begin{array}{c}\text { Barthelet } e t \\
\text { al., 1998a }\end{array}$ & \\
\hline \multirow[t]{2}{*}{3} & \multirow[t]{2}{*}{$\begin{array}{l}\text { BMRCa } \\
\text { BMRC }\end{array}$} & ML & $\begin{array}{l}\text { Equilibrium } 2 \times \mathrm{CO}_{2} \text { in mixed-layer } \\
\text { experiment }\end{array}$ & 2 & 60 & & 2.2 & & \multirow{2}{*}{\begin{tabular}{|c|} 
Colman and \\
McAvaney, \\
1995; Colman, \\
2001 \\
\end{tabular}} & \\
\hline & & CMIP2 & $1 \% \mathrm{CO}_{2}$ & 1 & 100 & 1.63 & & & & \\
\hline \multirow[t]{5}{*}{5} & \multirow[t]{5}{*}{$\begin{array}{l}\text { CCSR/NIES } \\
C C S R / N I E S\end{array}$} & ML & $\begin{array}{l}\text { Equilibrium } 2 \times \mathrm{CO}_{2} \text { in mixed-layer } \\
\text { experiment }\end{array}$ & 1 & 40 & & 3.6 & & \multirow[t]{5}{*}{$\begin{array}{l}\text { Emori et al., } \\
\quad 1999\end{array}$} & \\
\hline & & CMIP2 & $1 \% \mathrm{CO}_{2}$ & 1 & 80 & 1.8 & & & & \\
\hline & & G & $\begin{array}{l}\text { Historical equivalent } \mathrm{CO}_{2} \text { to } 1990 \text { then } \\
1 \% \mathrm{CO}_{2} \text { (approx. IS92a) }\end{array}$ & 1 & $1890-2099$ & & & & & \\
\hline & & GS & $\begin{array}{l}\text { As } \mathrm{G} \text { but including direct effect of } \\
\text { sulphate aerosols }\end{array}$ & 1 & $1890-2099$ & & & & & \\
\hline & & GS2 & $\begin{array}{l}1 \% \mathrm{CO}_{2}+\text { direct effect of sulphate } \\
\text { aerosols but with explicit representation }\end{array}$ & 1 & $1890-2099$ & & & & & \\
\hline \multirow[t]{6}{*}{31} & \multirow[t]{6}{*}{$\begin{array}{l}\text { CCSR/NIES2 } \\
\text { CCSR/NIES }\end{array}$} & ML & $\begin{array}{l}\text { Equilibrium } 2 \times \mathrm{CO}_{2} \text { in mixed-layer } \\
\text { experiment }\end{array}$ & 1 & 40 & & 5.1 & & \multirow[t]{6}{*}{$\begin{array}{c}\text { Nozawa et al., } \\
2001\end{array}$} & \\
\hline & & CMIP2 & $1 \% \mathrm{CO}_{2}$ & 1 & 80 & 3.1 & & 11.6 & & \\
\hline & & A1 & SRES A1 scenario & 1 & $1890-2100$ & & & & & \\
\hline & & $\mathrm{A} 2$ & SRES A2 scenario & 1 & $1890-2100$ & & & & & \\
\hline & & B1 & SRES B1 scenario & 1 & $1890-2100$ & & & & & \\
\hline & & $\mathrm{B} 2$ & SRES B2 scenario & 1 & $1890-2100$ & & & & & \\
\hline \multirow[t]{6}{*}{6} & \multirow[t]{6}{*}{$\begin{array}{l}\text { CGCM1 } \\
\text { CCCma }\end{array}$} & ML & $\begin{array}{l}\text { Equilibrium } 2 \times \mathrm{CO}_{2} \text { in mixed-layer } \\
\text { experiment }\end{array}$ & 1 & 30 & & 3.5 & & $\begin{array}{c}\text { Boer et al., } \\
1992 \\
\end{array}$ & \\
\hline & & CMIP2 & $1 \% \mathrm{CO}_{2}$ & 1 & 80 & 1.96 & & 3.6 & \multirow{5}{*}{$\begin{array}{l}\text { Boer et al., } \\
2000 \mathrm{a}, \mathrm{b}\end{array}$} & \multirow{5}{*}{$\begin{array}{l}1,000 \mathrm{yr} \\
\text { control }\end{array}$} \\
\hline & & $\mathrm{G}$ & $\begin{array}{l}\text { Historical equivalent } \mathrm{CO}_{2} \text { to } 1990 \text { then } \\
1 \% \mathrm{CO}_{2} \text { (approx. IS92a) }\end{array}$ & 1 & $1900-2100$ & & & & & \\
\hline & & GS & $\begin{array}{l}\text { As G but including direct effect of } \\
\text { sulphate aerosols }\end{array}$ & 3 & $1900-2100$ & & & & & \\
\hline & & GS2050 & $\begin{array}{l}\text { As GS but all forcings stabilised in } \\
\text { year } 2050\end{array}$ & 1 & $\begin{array}{l}1000 \text { after } \\
\text { stability }\end{array}$ & & & & & \\
\hline & & GS2100 & $\begin{array}{l}\text { As GS but all forcings stabilised in } \\
\text { year } 2100\end{array}$ & 1 & $\begin{array}{l}1000 \text { after } \\
\text { stability }\end{array}$ & & & & & \\
\hline \multirow[t]{3}{*}{7} & \multirow[t]{3}{*}{$\begin{array}{l}\text { CGCM2 } \\
\text { CCCma }\end{array}$} & GS & $\begin{array}{l}\text { Historical equivalent } \mathrm{CO}_{2} \text { to } 1990 \text { then } \\
1 \% \mathrm{CO}_{2} \text { (approx. IS } 92 \mathrm{a} \text { ) and direct } \\
\text { effect of sulphate aerosols }\end{array}$ & 3 & $1900-2100$ & & & & \multirow[t]{3}{*}{$\begin{array}{c}\text { Flato and } \\
\text { Boer, } 2001\end{array}$} & \multirow[t]{3}{*}{$\begin{array}{l}1,000 \mathrm{yr} \\
\text { control }\end{array}$} \\
\hline & & $\mathrm{A} 2$ & SRES A2 scenario & 3 & $1990-2100$ & & & & & \\
\hline & & B2 & \begin{tabular}{|l|} 
SRES B2 scenario \\
\end{tabular} & 3 & $1990-2100$ & & & & & \\
\hline \multirow[t]{7}{*}{10} & \multirow[t]{7}{*}{$\begin{array}{l}\text { CSIRO Mk2 } \\
\text { CSIRO }\end{array}$} & ML & $\begin{array}{l}\text { Equilibrium } 2 \times \mathrm{CO}_{2} \text { in mixed-layer } \\
\text { experiment }\end{array}$ & 1 & 60 & & 4.3 & & $\begin{array}{l}\text { Watterson } \text { et } \\
\text { al., } 1998\end{array}$ & \\
\hline & & CMIP2 & $1 \% \mathrm{CO}_{2}$ & 1 & 80 & 2.00 & & 3.7 & \multirow{2}{*}{$\begin{array}{c}\text { Gordon and } \\
\text { O'Farrell, } \\
1997\end{array}$} & \\
\hline & & $\mathrm{G}$ & \begin{tabular}{|l|} 
Historical equivalent $\mathrm{CO}_{2}$ to 1990 then \\
$1 \% \mathrm{CO}_{2}$ (approx. IS92a) \\
\end{tabular} & 1 & $1881-2100$ & & & & & \\
\hline & & G2080 & $\begin{array}{l}\text { As } \mathrm{G} \text { but forcing stabilised at } 2080(3 \times \\
\left.\text { initial } \mathrm{CO}_{2}\right)\end{array}$ & 1 & $\begin{array}{l}700 \text { after } \\
\text { stability }\end{array}$ & & & & Hirst, 1999 & \\
\hline & & GS & $\begin{array}{l}\text { As } G+\text { direct effect of sulphate } \\
\text { aerosols }\end{array}$ & 1 & $1881-2100$ & & & & \begin{tabular}{|c|} 
Gordon and \\
O'Farrell, 1997 \\
\end{tabular} & \\
\hline & & $\mathrm{A} 2$ & SRES A2 scenario & 1 & $1990-2100$ & & & & & \\
\hline & & $\mathrm{B} 2$ & SRES B2 scenario & 1 & $1990-2100$ & & & & & \\
\hline \multirow[t]{2}{*}{11} & \multirow[t]{2}{*}{$\begin{array}{l}\text { CSM } 1.0 \\
\text { NCAR }\end{array}$} & ML & $\begin{array}{l}\text { Equilibrium } 2 \times \mathrm{CO}_{2} \text { in mixed-layer } \\
\text { experiment }\end{array}$ & 1 & 50 & & 2.1 & & $\begin{array}{l}\text { Meehl et al., } \\
\text { 2000a }\end{array}$ & \\
\hline & & CMIP2 & $1 \% \mathrm{CO}_{2}$ & 1 & 80 & 1.43 & & 1.9 & & \\
\hline 12 & $\begin{array}{l}\text { CSM } 1.3^{\mathrm{a}} \\
\text { NCAR }\end{array}$ & GS & $\begin{array}{l}\text { Historical GHGs + direct effect of sulph- } \\
\text { ate aerosols to } 1990 \text { then BAU } \mathrm{CO}_{2}+ \\
\text { direct effect of sulphate aerosols includ- } \\
\text { ing effects of pollution control policies } \\
\end{array}$ & 1 & $1870-2100$ & & & & \begin{tabular}{|l} 
Boville et al., \\
$2001 ;$ \\
Dai et al. \\
2001
\end{tabular} & \\
\hline & & GS2150 & $\begin{array}{l}\text { Historical GHGs + direct effect of } \\
\text { sulphate to aerosols to } 1990 \text { then as GS } \\
\text { except WRE550 scenario for } \mathrm{CO}_{2} \text { until } \\
\text { it reaches } 550 \mathrm{ppm} \text { in } 2150\end{array}$ & 1 & $1870-2100$ & & & & & \\
\hline & & $\mathrm{A} 1$ & SRES A1 scenario & 1 & $1870-2100$ & & & & & \\
\hline & & A2 & SRES A2 scenario & 1 & $1870-2100$ & & & & & \\
\hline & & $\mathrm{B} 2$ & SRES B2 scenario & 1 & $1870-2100$ & & & & & \\
\hline & & CMIP2 & $1 \% \mathrm{CO}_{2}$ & 1 & 100 & 1.58 & & 2.2 & & \\
\hline 14 & $\begin{array}{l}\text { ECHAM3/LSG } \\
D K R Z\end{array}$ & G & $\begin{array}{l}\text { Historical equiv } \mathrm{CO}_{2} \text { to } 1990 \text { then } 1 \% \\
\mathrm{CO}_{2} \text { (approx. IS92a) }\end{array}$ & 1 & $1881-2085$ & & & & \begin{tabular}{|c|} 
Cubasch et al., \\
1992, 1994,
\end{tabular} & \\
\hline & & G2050 & $\begin{array}{l}\text { As } \mathrm{G} \text { but forcing stabilised at } 2050(2 \times \\
\left.\text { initial } \mathrm{CO}_{2}\right)\end{array}$ & 1 & $\begin{array}{l}850 \text { after } \\
\text { stability }\end{array}$ & & & & 1996 & \\
\hline & & G2110 & $\begin{array}{l}\text { As } \mathrm{G} \text { but forcing stabilised at } 2110(4 \times \\
\left.\text { initial } \mathrm{CO}_{2}\right)\end{array}$ & 2 & $\begin{array}{l}850 \text { after } \\
\text { stability }\end{array}$ & & & & $\begin{array}{c}\text { Voss and } \\
\text { Mikolajewicz, }\end{array}$ & $\begin{array}{l}\text { Periodically } \\
\text { synchronous }\end{array}$ \\
\hline & & GS & As G + direct effect of sulphate aerosols & 2 & $1881-2050$ & & & & 2001 & coupling \\
\hline & & ML & $\begin{array}{l}\text { Equilibrium } 2 \times \mathrm{CO}_{2} \text { in mixed-layer } \\
\text { experiment }\end{array}$ & 1 & 60 & & 3.2 & & \begin{tabular}{|c|} 
Cubasch et al., \\
$1992,1994,1996 \mathrm{~b}$
\end{tabular} & \\
\hline
\end{tabular}

${ }^{a}$ CSM 1.3 was at the time of the printing of this report not archived completely in the DDC. It is therefore not considered in calculations and diagrams refering to the DDC experiments with the exception of Figure 9.5. 
Table 9.1: Continuation.

\begin{tabular}{|c|c|c|c|c|c|c|c|c|c|c|}
\hline $\begin{array}{c}\text { Model } \\
\text { Number } \\
\text { (see } \\
\text { Chapter 8, } \\
\text { Table 8.1) }\end{array}$ & $\begin{array}{l}\text { Model Name and } \\
\text { centre in italics } \\
\text { (see Chapter } 8 \\
\text { Table 8.1) }\end{array}$ & Scenario name & Scenario description & $\begin{array}{l}\text { Number of } \\
\text { simulations }\end{array}$ & $\begin{array}{l}\text { Length of } \\
\text { simulation or } \\
\text { starting and } \\
\text { final year }\end{array}$ & $\begin{array}{l}\text { Transient } \\
\text { Climate } \\
\text { Response } \\
\text { (TCR) } \\
\text { (Section } \\
9.2 .1)\end{array}$ & $\begin{array}{c}\text { Equilibrium } \\
\text { climate } \\
\text { sensitivity } \\
\text { (Section } 9.2 .1 \text { ) } \\
\text { (in bold used in } \\
\text { Figure. } 9.18 \text { / } \\
\text { Table } 9.4\end{array}$ & $\begin{array}{c}\text { Effective } \\
\text { climate } \\
\text { sensitivity } \\
\text { (Section } \\
9.2 .1 \text { ) (from } \\
\text { CMIP2 yrs } \\
\text { 61-80) in bold } \\
\text { used in } \\
\text { Table A1 }\end{array}$ & References & Remarks \\
\hline \multirow[t]{6}{*}{15} & \multirow{6}{*}{$\begin{array}{l}\text { ECHAM4/OPYC } \\
M P I\end{array}$} & CMIP2 & $1 \% \mathrm{CO}_{2}$ & 1 & 80 & 1.4 & & 2.6 & \multirow{4}{*}{$\begin{array}{c}\text { Roeckner } \text { et } \\
\text { al., } 1999\end{array}$} & \\
\hline & & $\mathrm{G}$ & Historical GHGs to 1990 then IS92a & 1 & $1860-2099$ & & & & & \\
\hline & & GS & $\begin{array}{l}\text { As } G+\text { direct effect of sulphate } \\
\text { aerosol interactively calculated }\end{array}$ & 1 & $1860-2049$ & & & & & \\
\hline & & GSIO & $\begin{array}{l}\text { As GS + indirect effect of sulphate } \\
\text { aerosol + ozone }\end{array}$ & 1 & $1860-2049$ & & & & & \\
\hline & & $\mathrm{A} 2$ & SRES A2 scenario & 1 & $1990-2100$ & & & & \multirow{2}{*}{$\begin{array}{l}\text { Stendel et al., } \\
2000\end{array}$} & \\
\hline & & B2 & SRES B2 scenario & 1 & $1990-2100$ & & & & & \\
\hline \multirow[t]{6}{*}{16} & \multirow[t]{6}{*}{$\begin{array}{l}\text { GFDL_R15_a } \\
G F D L\end{array}$} & ML & \begin{tabular}{|l|}
$\begin{array}{l}\text { Equilibrium } 2 \times \mathrm{CO}_{2} \text { in mixed-layer } \\
\text { experiment }\end{array}$ \\
\end{tabular} & 2 & 40 & & $\begin{array}{c}3.7 \\
(3.9)^{\mathrm{b}}\end{array}$ & & \begin{tabular}{|c|}
$\begin{array}{c}\text { Manabe } \text { et al. } \\
1991\end{array}$ \\
\end{tabular} & \multirow[t]{6}{*}{$\begin{array}{c}15,000 \text { year } \\
\text { control }\end{array}$} \\
\hline & & CMIP2 & $1 \% \mathrm{CO}_{2}$ & 2 & 80 & 2.15 & & 4.2 & \multirow{3}{*}{$\begin{array}{c}\text { Stouffer and } \\
\text { Manabe, } 1999\end{array}$} & \\
\hline & & CMIP270 & \begin{tabular}{|l|} 
As CMIP2 but forcing stabilised at \\
year $70\left(2 \times\right.$ initial $\left.\mathrm{CO}_{2}\right)$ \\
\end{tabular} & 1 & 4000 & & $(4.5)^{\mathrm{c}}$ & & & \\
\hline & & CMIP2140 & $\begin{array}{l}\text { As CMIP2 but forcing stabilised at } \\
\text { year } 140\left(4 \times \text { initial } \mathrm{CO}_{2}\right) \\
\end{array}$ & 1 & 5000 & & & & & \\
\hline & & G & $\begin{array}{l}\text { Historical equivalent } \mathrm{CO}_{2} \text { to } 1990 \text { then } \\
1 \% \mathrm{CO}_{2} \text { (approximate IS92a) } \\
\end{array}$ & 1 & $1766-2065$ & & & & \multirow{2}{*}{$\begin{array}{l}\text { Haywood } e t \\
\text { al., 1997; } \\
\text { Sarmiento et } \\
\text { al., } 1998 \\
\end{array}$} & \\
\hline & & GS & As $G+$ direct effect of sulphate aerosols & 2 & $1766-2065$ & & & & & \\
\hline \multirow[t]{2}{*}{17} & \multirow[t]{2}{*}{$\begin{array}{l}\text { GFDL_R15_b } \\
\text { GFDL }\end{array}$} & CMIP2 & $1 \% \mathrm{CO}_{2}$ & 1 & 80 & $\begin{array}{c}\text { Data } \\
\text { unavailable } \\
\end{array}$ & & & & \\
\hline & & GS & $\begin{array}{l}\text { Historical equivalent } \mathrm{CO}_{2} \text { to } 1990 \text { then } \\
1 \% \mathrm{CO}_{2} \text { (approximate IS92a) + direct } \\
\text { effect of sulphate aerosols }\end{array}$ & $\begin{array}{l}3 \\
3 \\
3\end{array}$ & $\begin{array}{l}1766-2065 \\
1866-2065 \\
1916-2065\end{array}$ & & & & $\begin{array}{l}\text { Dixon and } \\
\text { Lanzante, } \\
1999\end{array}$ & \\
\hline \multirow[t]{7}{*}{18} & \multirow[t]{7}{*}{$\begin{array}{l}\text { GFDL_R30_c } \\
\text { GFDL }\end{array}$} & ML & \begin{tabular}{|l|}
$\begin{array}{l}\text { Equilibrium } 2 \times \mathrm{CO}_{2} \text { in mixed-layer } \\
\text { experiment }\end{array}$ \\
\end{tabular} & 1 & 40 & & 3.4 & & & \multirow{2}{*}{$\begin{array}{c}2 \times 1,000 \text { year } \\
\text { control runs } \\
\text { with different } \\
\text { oceanic dia- } \\
\text { pycnal mixing }\end{array}$} \\
\hline & & CMIP2 & $1 \% \mathrm{CO}_{2}$ & 2 & 80 & 1.96 & & & & \\
\hline & & CMIP270 & $\begin{array}{l}\text { As CMIP2 but forcing stabilised at } \\
\text { year } 70\left(2 \times \text { initial } \mathrm{CO}_{2}\right)\end{array}$ & 1 & $\begin{array}{l}140 \text { after } \\
\text { stability }\end{array}$ & & & & & \multirow{2}{*}{\begin{tabular}{|c|} 
Different \\
oceanic \\
diapycnal \\
mixing
\end{tabular}} \\
\hline & & CMIP2140 & $\begin{array}{l}\text { As CMIP2 but forcing stabilised at } \\
\text { year } 140\left(4 \times \text { initial } \mathrm{CO}_{2}\right)\end{array}$ & 1 & $\begin{array}{l}160 \text { after } \\
\text { stability }\end{array}$ & & & & & \\
\hline & & GS & $\begin{array}{l}\text { Historical equivalent } \mathrm{CO}_{2} \text { to } 1990 \text { then } \\
1 \% \mathrm{CO} \text { (approximate IS92a) + direct } \\
\text { effect of sulphate aerosols }\end{array}$ & 9 & $1866-2090$ & & & & \begin{tabular}{|c|} 
Knutson et al., \\
1999
\end{tabular} & \\
\hline & & $\mathrm{A} 2$ & SRES A2 scenario & 1 & 1960-2090 & & & & & \\
\hline & & $\mathrm{B} 2$ & SRES B2 scenario & 1 & $1960-2090$ & & & & & \\
\hline \multirow[t]{2}{*}{20} & \multirow[t]{2}{*}{$\begin{array}{l}\text { GISS2 } \\
\text { GISS }\end{array}$} & ML & \begin{tabular}{|l|}
$\begin{array}{l}\text { Equilibrium } 2 \times \mathrm{CO}_{2} \text { in mixed-layer } \\
\text { experiment }\end{array}$ \\
\end{tabular} & 1 & 40 & & $(3.1)^{d}$ & & $\begin{array}{l}\text { Yao and Del } \\
\text { Genio, } 1999 \\
\end{array}$ & \\
\hline & & CMIP2 & $1 \% \mathrm{CO}_{2}$ & 1 & 80 & 1.45 & & & $\begin{array}{c}\text { Russell } \text { et al., } \\
\text { 1995; Russell } \\
\text { and Rind, 1999 }\end{array}$ & \\
\hline 21 & \begin{tabular}{|l} 
GOALS \\
IAP/LASG
\end{tabular} & CMIP2 & $1 \% \mathrm{CO}_{2}$ & 1 & 80 & 1.65 & & & & \\
\hline \multirow[t]{6}{*}{22} & \multirow[t]{6}{*}{$\begin{array}{l}\text { HadCM2 } \\
U K M O\end{array}$} & ML & $\begin{array}{l}\text { Equilibrium } 2 \times \mathrm{CO}_{2} \text { in mixed-layer } \\
\text { experiment }\end{array}$ & 1 & 40 & & 4.1 & & \begin{tabular}{c|} 
Senior and \\
Mitchell, 2000 \\
\end{tabular} & \\
\hline & & CMIP2 & $1 \% \mathrm{CO}_{2}$ & 1 & 80 & 1.7 & & 2.5 & \begin{tabular}{c|} 
Keen and \\
Murphy, 1997 \\
\end{tabular} & \multirow[t]{5}{*}{$\begin{array}{l}1,000 \text { year } \\
\text { control run }\end{array}$} \\
\hline & & CMIP270 & $\begin{array}{l}\text { As CMIP2 but forcing stabilised at } \\
\text { year } 70\left(2 \times \text { initial } \mathrm{CO}_{2}\right) \\
\end{array}$ & 1 & $\begin{array}{l}900 \text { after } \\
\text { stability }\end{array}$ & & & & \begin{tabular}{|c|} 
Senior and \\
Mitchell, 2000 \\
\end{tabular} & \\
\hline & & $\mathrm{G}$ & $\begin{array}{l}\text { Historical equivalent } \mathrm{CO}_{2} \text { to } 1990 \\
\text { then } 1 \% \mathrm{CO}_{2} \text { (approximate IS92a) }\end{array}$ & 4 & $1881-2085$ & & & & \begin{tabular}{|c|} 
Mitchell et al., \\
1995; Mitchell \\
and Johns, 1997 \\
\end{tabular} & \\
\hline & & G2150 & $\begin{array}{l}\text { As G but all forcings stabilised in } \\
\text { year } 2150\end{array}$ & 1 & $\begin{array}{l}110 \text { after } \\
\text { stability }\end{array}$ & & & & \begin{tabular}{|l|} 
Mitchell et al. \\
2000
\end{tabular} & \\
\hline & & GS & $\begin{array}{l}\text { As } G+\text { direct effect of sulphate } \\
\text { aerosols }\end{array}$ & 4 & $1860-2100$ & & & & \begin{tabular}{|} 
Mitchell et al., \\
1995; Mitchell \\
and Johns, 1997 \\
\end{tabular} & \\
\hline \multirow[t]{5}{*}{23} & \multirow[t]{5}{*}{$\begin{array}{l}\text { HadCM3 } \\
\text { UKMO }\end{array}$} & ML & \begin{tabular}{|l}
$\begin{array}{l}\text { Equilibrium } 2 \times \mathrm{CO}_{2} \text { in mixed-layer } \\
\text { experiment }\end{array}$ \\
\end{tabular} & 1 & 30 & & 3.3 & & $\begin{array}{l}\text { Williams } \text { et al., } \\
2001\end{array}$ & \\
\hline & & CMIP2 & $1 \% \mathrm{CO}_{2}$ & 1 & 80 & 2.0 & & 3.0 & & \multirow{4}{*}{$\begin{array}{l}1,800 \text { year } \\
\text { control run }\end{array}$} \\
\hline & & G GSIO & $\begin{array}{l}\text { Historical GHGs to } 1990 \text { then IS95a } \\
\begin{array}{l}\text { As G + direct and indirect effect of } \\
\text { sulphate aerosols + ozone changes }\end{array} \\
\end{array}$ & 1 & $\begin{array}{l}1860-2100 \\
1860-2100\end{array}$ & & & & \multirow[t]{3}{*}{\begin{tabular}{|c|} 
Mitchell et al., \\
1998; Gregory \\
and Lowe, 2000 \\
Johns et al., \\
2001
\end{tabular}} & \\
\hline & & $\mathrm{A} 2$ & SRES A2 scenario & 1 & $1990-2100$ & & & & & \\
\hline & & $\mathrm{B} 2$ & SRES B2 scenario & 1 & $1990-2100$ & & & & & \\
\hline
\end{tabular}

${ }^{\mathrm{b}}$ The equilibrium climate sensitivity if the control SSTs from the coupled model are used.

${ }^{c}$ The equilibrium climate sensitivity calculated from the coupled model.

$\mathrm{d}$ The ML experiment used in Table 9.2 for the GISS model were performed with a different atmospheric model to that used in the coupled model listed here. 
Table 9.1: Continuation.

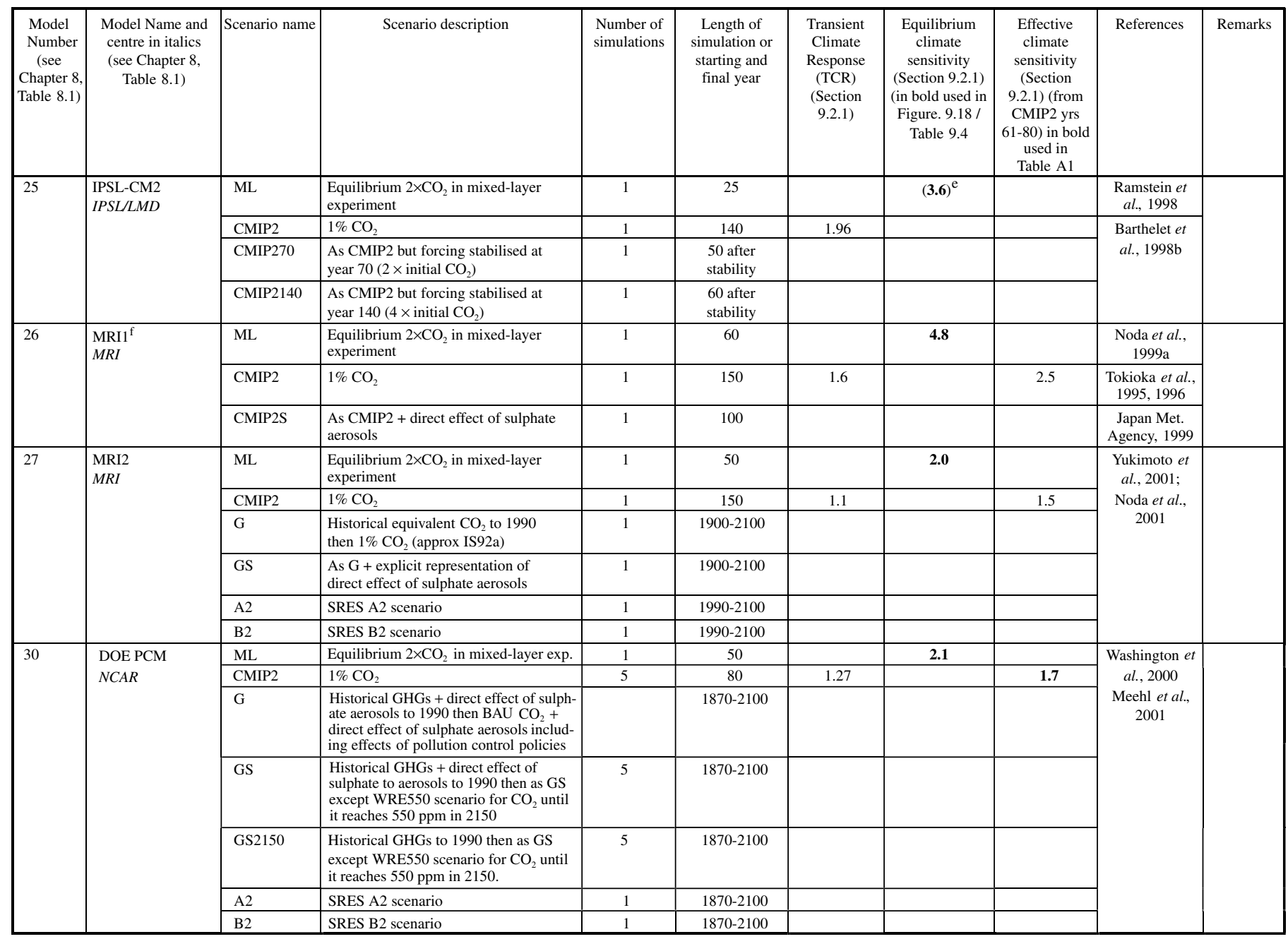

${ }^{\mathrm{e}}$ The ML experiment used in Table 9.2 for the IPSL-CM2 model were performed with a slightly earlier version of the atmospheric model than that used in the coupled model, but tests have suggested the changes would not affect the equilibrium climate sensitivity.

${ }^{\mathrm{f}}$ Model MRI1 exists in two versions. At the time of writing, more complete assessment data was available for the earlier version, whose control run is in the CMIP1 database. This model is used in Chapter 8. The model used in Chapter 9 has two extra ocean levels and a modified ocean mixing scheme. Its control run is in the CMIP2 database. The equilibrium climate sensitivities and Transient Climate Responses (shown in this table) of the two models are the same.
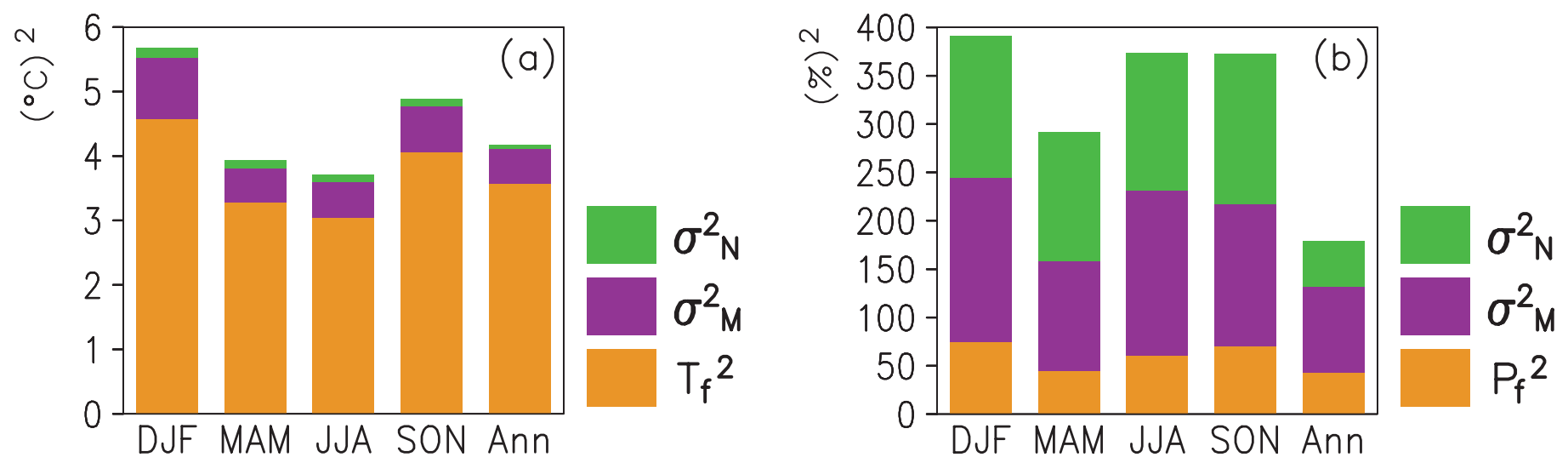

Figure 9.4: Intercomparison statistics for seasonal and annual (a) temperature and (b) precipitation changes in nineteen CMIP2 experiments at the doubling of $\mathrm{CO}_{2}$ (years 61 to 80). The total length of the bars shows the mean squared amplitude of the simulated local temperature and precipitation changes averaged over all experiments and over the whole world. The lowermost part of each bar represents a nominally unbiased "common signal", the mid-part directly model-related variance and the top part the inter-experiment variance attributed to internal variability. Precipitation changes are defined as $100 \% \times\left(\mathrm{P}_{\mathrm{G}}-\mathrm{P}_{\mathrm{CTRL}}\right) / \mathrm{Max}\left(\mathrm{P}_{\mathrm{CTRL}}, 0.25 \mathrm{~mm} /\right.$ day $)$, where the lower limit of $0.25 \mathrm{~mm} /$ day is used to reduce the sensitivity of the global statistics to areas with very little control run precipitation. 
(a)
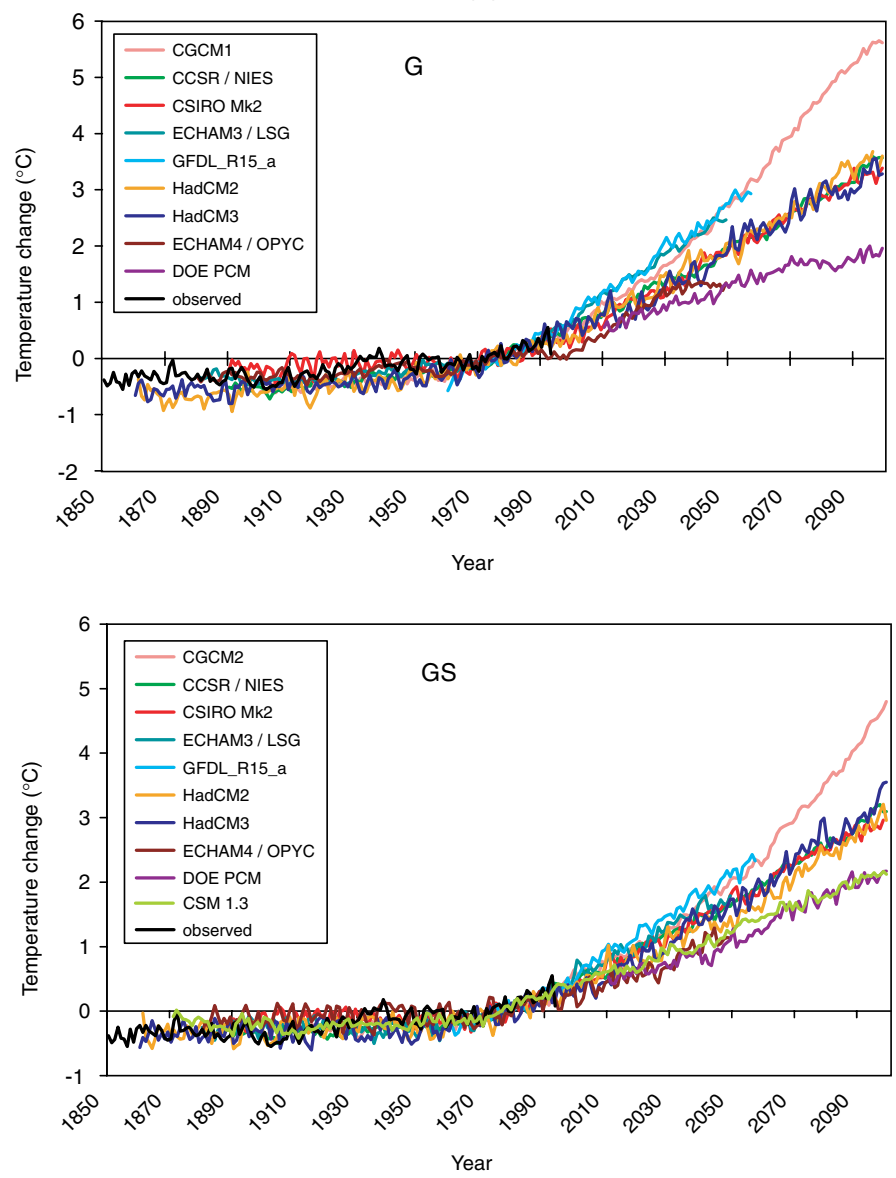

(b)
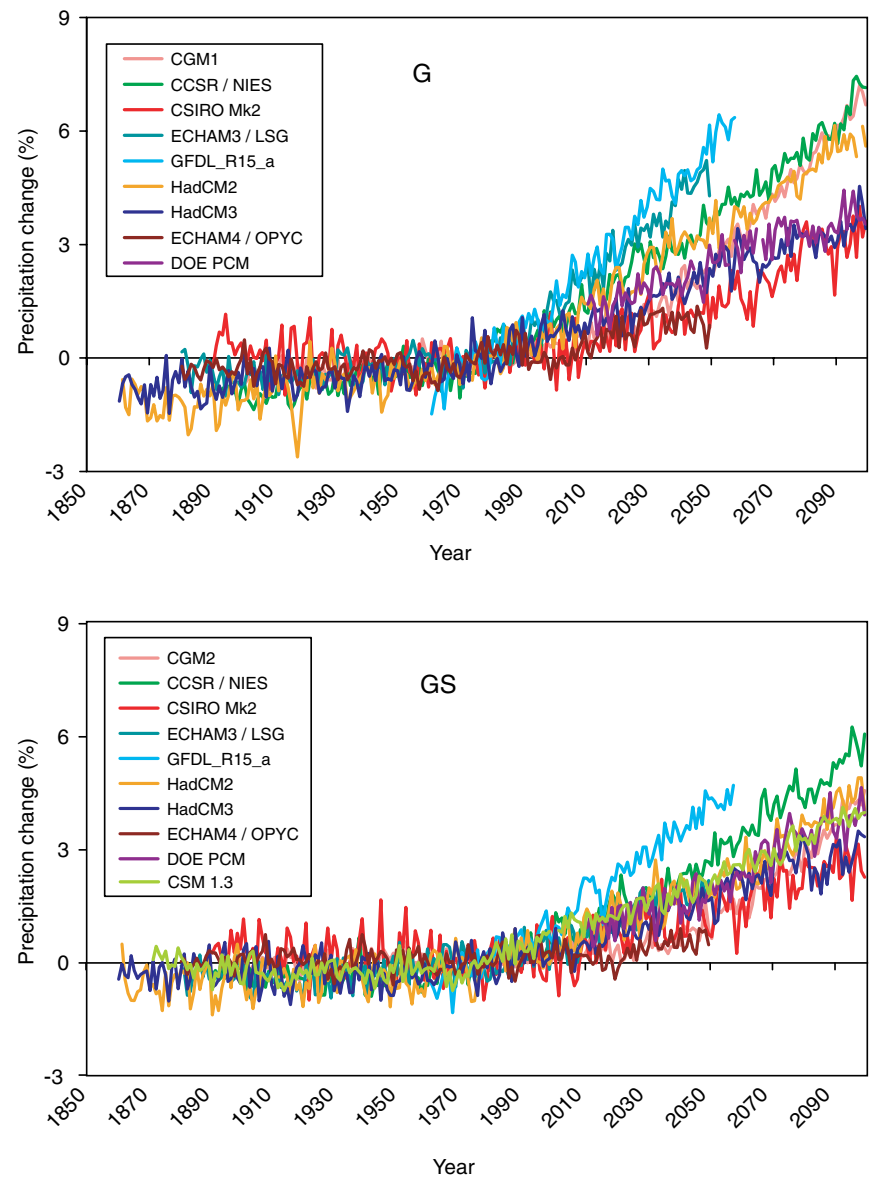

Figure 9.5: (a) The time evolution of the globally averaged temperature change relative to the years (1961 to 1990) of the DDC simulations (IS92a). G: greenhouse gas only (top), GS: greenhouse gas and sulphate aerosols (bottom). The observed temperature change (Jones, 1994) is indicated by the black line. (Unit: ${ }^{\circ} \mathrm{C}$ ). See Table 9.1 for more information on the individual models used here. (b) The time evolution of the globally averaged precipitation change relative to the years (1961 to 1990) of the DDC simulations. GHG: greenhouse gas only (top), GS: greenhouse gas and sulphate aerosols (bottom). (Unit: \%). See Table 9.1 for more information on the individual models used here.

the annual mean to about $50 \%$ in individual seasons. Thus there is more internal variability and model differences and less common signal indicating lower reliability in the changes of precipitation compared to temperature.

\subsubsection{Projections of future climate from forcing scenario experiments (IS92a)}

Please note that the use of projections for forming climate scenarios to study the impacts of climate change is discussed in Chapter 13.

These experiments include changes in greenhouse gases plus the direct effect of sulphate aerosol using IS92a type forcing (see Chapter 6 for a complete discussion of direct and indirect effect forcing from sulphate aerosols). The temperature change (Figures 9.5a and 9.7a, top) for the 30-year average 2021 to 2050 compared with 1961 to 1990 is $+1.3^{\circ} \mathrm{C}$ with a range of +0.8 to $+1.7^{\circ} \mathrm{C}$ as opposed to $+1.6^{\circ} \mathrm{C}$ with a range of +1.0 to $+2.1^{\circ} \mathrm{C}$ for greenhouse gases only (Cubasch and Fischer-Bruns, 2000). The experiments including sulphate aerosols show a smaller temperature rise compared to experiments without sulphate aerosols due to the negative radiative forcing of these aerosols. Additionally, in these simulations $\mathrm{CO}_{2}$ would double around year 2060. Thus for the averaging period being considered, years 2021 to 2050, the models are still short of the $\mathrm{CO}_{2}$ doubling point seen in the idealised $1 \% / \mathrm{yr} \quad \mathrm{CO}_{2}$ increase simulations. These sensitivity ranges could be somewhat higher (about 30\%) if the positive feedback effects from the carbon cycle are included interactively but the magnitude of these feedbacks is uncertain (Cox et al., 2000; Friedlingstein, 2001). The globally averaged precipitation response for 2021 to 2050 for greenhouse gases plus sulphates is $+1.5 \%$ with a range of +0.5 to $+3.3 \%$ as opposed to $+2.3 \%$ with a range of +0.9 to $+4.4 \%$ for greenhouse gases only (Figures 9.5b and 9.7a, bottom).

\subsubsection{Marker scenario experiments (SRES)}

As discussed in Section 9.1.2, only the draft marker SRES scenarios A2 and B2 have been integrated with more than one AOGCM, because the scenarios were defined too late to have experiments ready from all the modelling groups in time for this report. Additionally, some new versions of models have been 
(a)
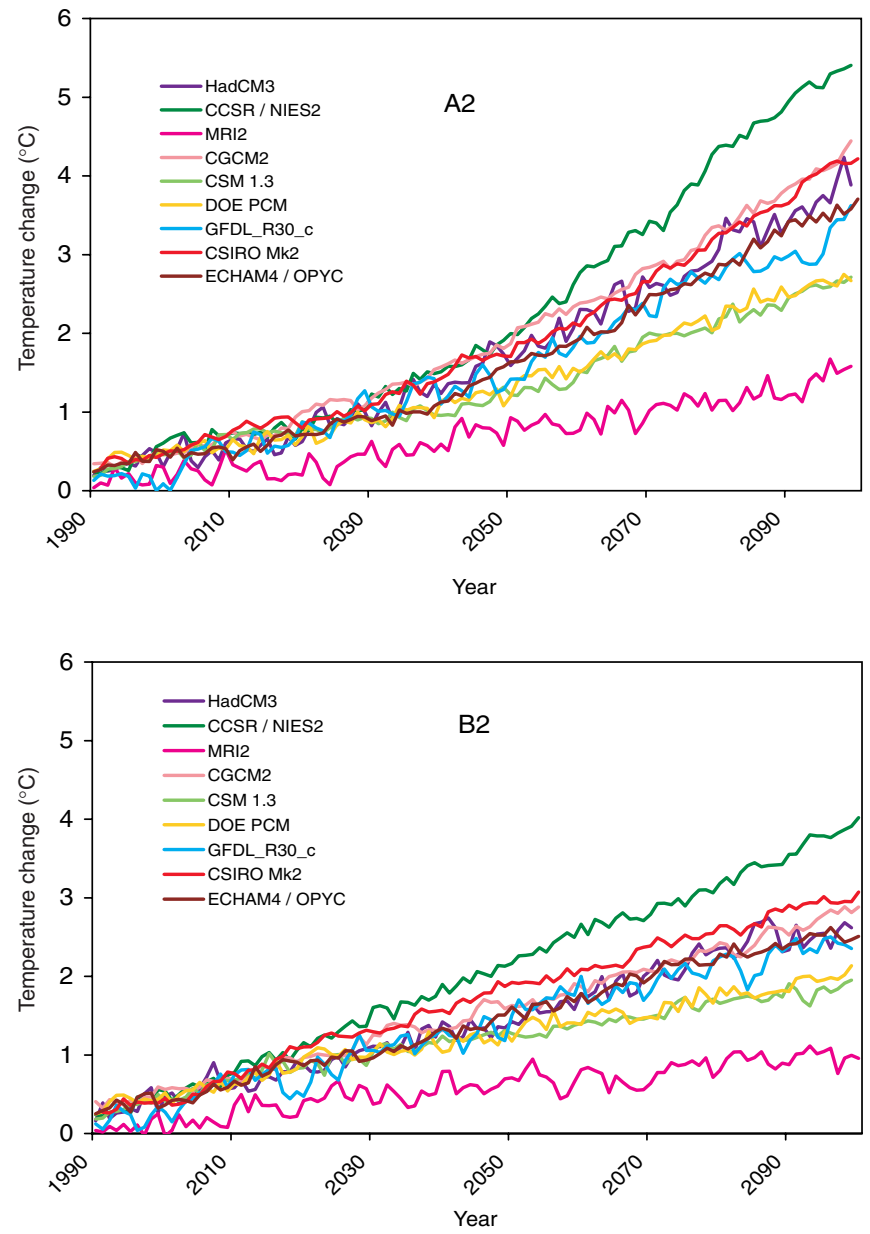

(b)
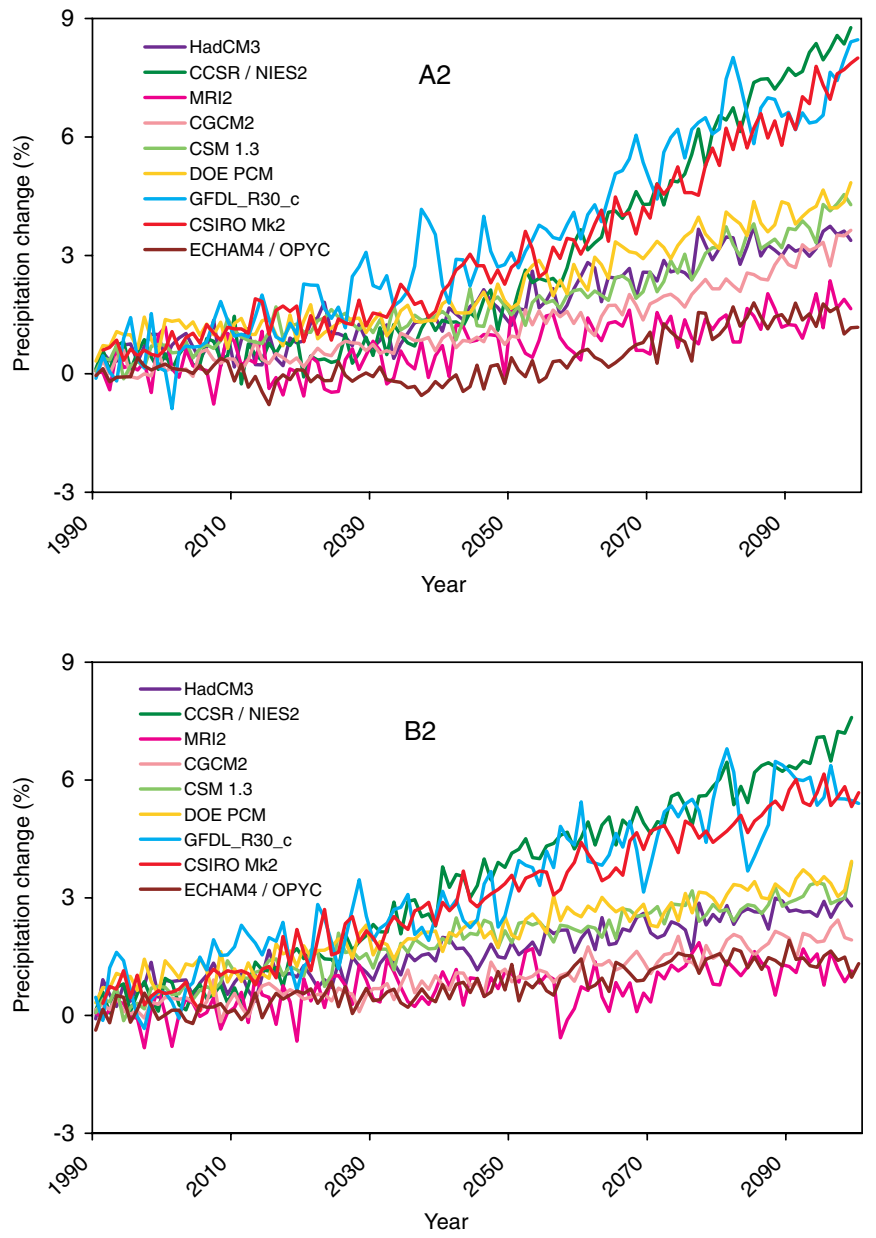

Figure 9.6: (a) The time evolution of the globally averaged temperature change relative to the years (1961 to 1990) of the SRES simulations A2 (top) and B2 (bottom) (Unit: ${ }^{\circ} \mathrm{C}$ ). See Table 9.1 for more information on the individual models used here. (b) The time evolution of the globally averaged precipitation change relative to the years (1961 to 1990) of the SRES simulations A2 (top) and B2 (bottom) (Unit: \%). See Table 9.1 for more information on the individual models used here.

used to run the $\mathrm{A} 2$ and $\mathrm{B} 2$ scenarios that have not had time to be evaluated by Chapter 8 . Therefore, we present results from all the model simulations and consider them all as possible realisations of future climate change, but their ranges are not directly comparable to the simple model results in Section 9.3.3 (range: 1.4 to $5.8^{\circ} \mathrm{C}$ ), because in the simple model analysis seven somewhat different versions of the nine models have been considered. Additionally, for the AOGCMs the temperature changes are evaluated for an average of years 2071 to 2100 compared with 1961 to 1990 , while the simple model results are differences of the year 2100 minus 1990.

The average temperature response from nine AOGCMs using the SRES A2 forcing (Figures 9.6a and 9.7b, top) for the 30-year average 2071 to 2100 relative to 1961 to 1990 is $+3.0^{\circ} \mathrm{C}$ with a range of +1.3 to $+4.5^{\circ} \mathrm{C}$, while using the SRES B2 scenarios it amounts to $+2.2^{\circ} \mathrm{C}$ with a range of +0.9 to $+3.4^{\circ} \mathrm{C}$. The B2 scenario produces a smaller warming which is consistent with its lower positive radiative forcing at the end of the 21st century. For the 30-year average 2021 to 2050 using the A2 scenario, the globally averaged surface air temperature increase compared to 1961 with 1990 is $+1.1^{\circ} \mathrm{C}$ with a range of +0.5 to $+1.4^{\circ} \mathrm{C}$, while using the SRES B2 scenarios it amounts to $+1.2^{\circ} \mathrm{C}$ with a range of +0.5 to $+1.7^{\circ} \mathrm{C}$. The values for the SRES scenarios for the mid-21st century are lower than for the IS92a scenarios for the corresponding period due to differences in the forcing.

The average precipitation response using the SRES A2 forcing (Figures 9.6b and 9.7b, bottom) for the 30-year average 2071 to 2100 compared with 1961 to 1990 is an increase of $3.9 \%$ with a range of 1.3 to $6.8 \%$, while using the SRES B2 scenarios it amounts to an increase of $3.3 \%$ with a range of 1.2 to $6.1 \%$. The lower precipitation increase values for the B2 scenario are consistent with less globally averaged warming for that scenario at the end of the 21 st century compared with A2. For the 30-year average 2021 to 2050 the globally averaged precipitation increases $1.2 \%$ for the $\mathrm{A} 2$ scenario, and $1.6 \%$ for $\mathrm{B} 2$ which is again consistent with the slightly greater global warming in B2 for mid-21st century compared with A2. Globally averaged 
(a)

Global temperature change $\left({ }^{\circ} \mathrm{C}\right)$

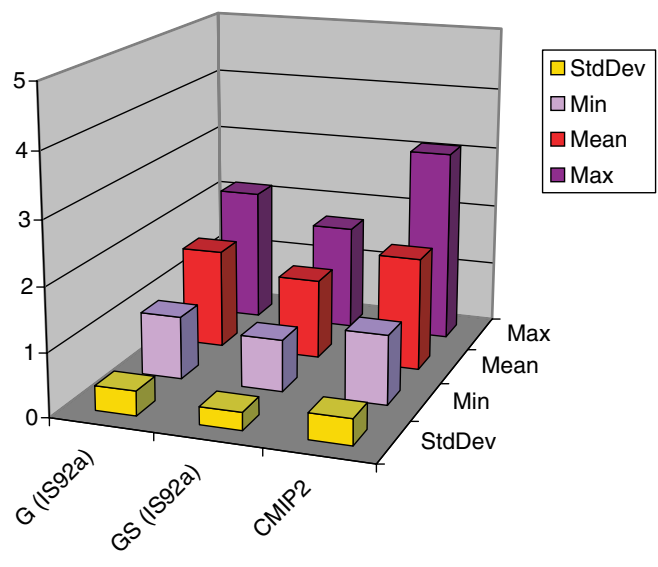

Global precipitation change (\%)

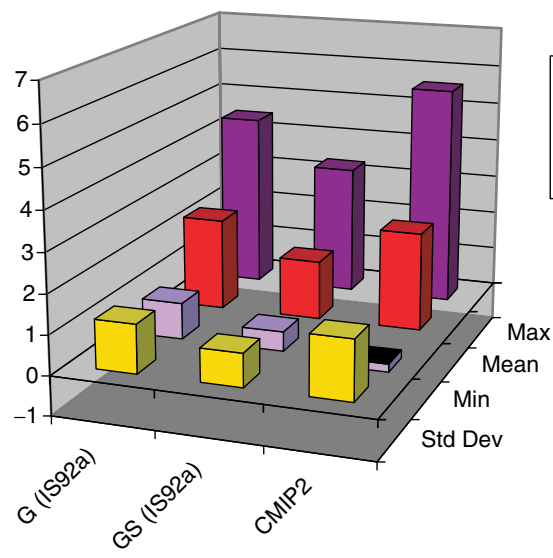

(b)

Global temperature change $\left({ }^{\circ} \mathrm{C}\right)$

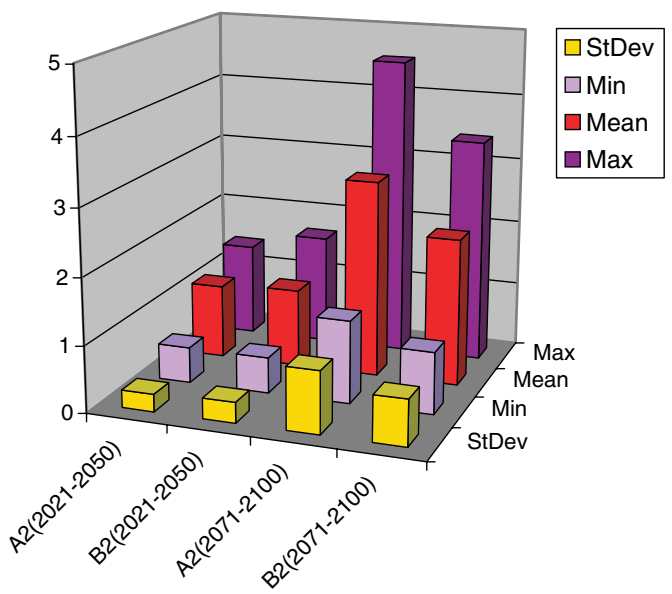

Global precipitation change (\%)

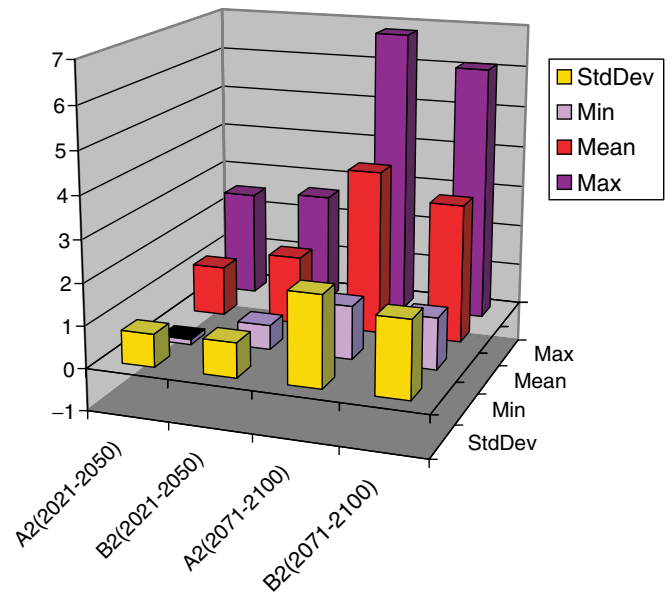

Figure 9.7: (a) The global mean, the maximum and minimum simulated by the respective models and the standard deviation for the CMIP2 experiments at the time of $\mathrm{CO}_{2}$-doubling and for the DDC experiments during the years 2021 to 2050 relative to the years 1961 to 1990 for temperature (top) (Unit: ${ }^{\circ} \mathrm{C}$ ) and precipitation (bottom) (Unit: \%). G: greenhouse gases only, GS: greenhouse gases and sulphate aerosols. See Table 9.1 for more information on the individual models used here. (b) The global mean, the maximum and minimum simulated by the respective models and the standard deviation for the SRES scenario experiments A2 and B2 performed by the AOGCMs, for the years 2021 to 2050 and 2071 to 2100 relative to the years 1961 to 1990 for temperature (top) (Unit: ${ }^{\circ} \mathrm{C}$ ) and precipitation (bottom) (Unit: \%). See Table 9.1 for more information on the individual models used here.

changes of temperature and precipitation are summarised in Figure 9.7b. A more extensive analysis of globally averaged temperature changes for a wider range of SRES forcing scenarios using a simple climate model is given in Section 9.3.3.

\subsubsection{Patterns of Future Climate Change}

For the change in annual mean surface air temperature in the various cases, the model experiments show the familiar pattern documented in the SAR with a maximum warming in the high latitudes of the Northern Hemisphere and a minimum in the Southern Ocean (due to ocean heat uptake) evident in the zonal mean for the CMIP2 models (Figure 9.8) and the geographical patterns for all categories of models (Figure 9.10). For the zonal means in Figure 9.8 there is consistent mid-tropospheric tropical warming and stratospheric cooling. The range tends to increase with height (Figure 9.8, middle) partly due to the variation in the level of the tropopause among the models. Ocean heat uptake also contributes to a minimum of warming in the North Atlantic, while land warms more rapidly than ocean almost everywhere (Figure 9.10). The large warming in high latitudes of the Northern Hemisphere is connected with a reduction in the snow (not shown) and sea-ice cover (Figure 9.9).

The ensemble mean temperature divided by its standard deviation $\{\Delta \mathrm{T}\} / \sigma_{\{\Delta \mathrm{T}\}}$ provides a measure of the consistency of the climate change patterns (Section 9.2). Different types and 

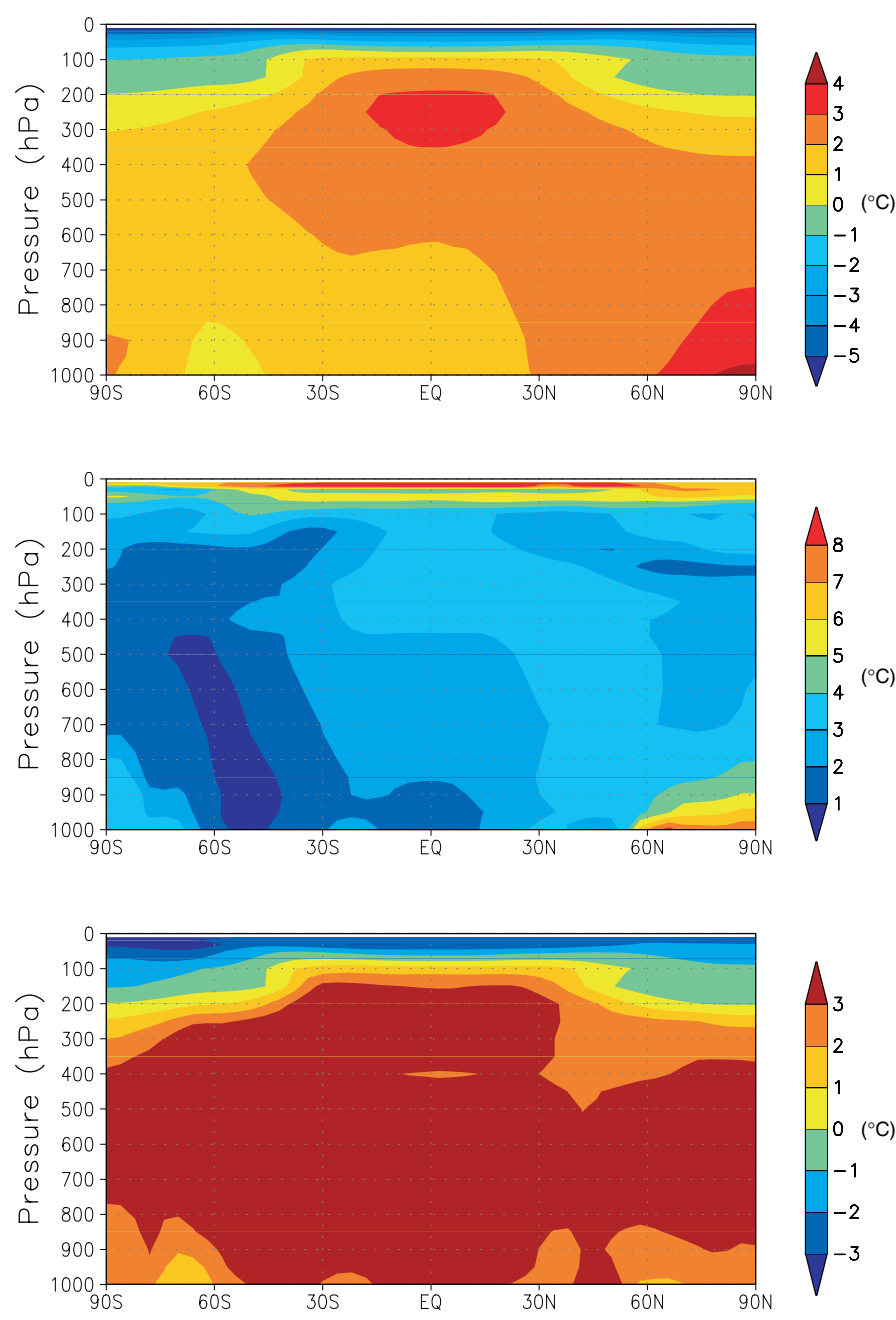

Figure 9.8: Multi-model annual mean zonal temperature change (top), zonal mean temperature change range (middle) and the zonal mean change divided by the multi-model standard deviation of the mean change (bottom) for the CMIP2 simulations. See text for scenario definitions and description of analysis technique. (Unit: ${ }^{\circ} \mathrm{C}$ ).

different numbers of models enter the ensembles for the G, GS and SRES A2 and B2 cases and results will depend both on this and on the difference in forcing. Values greater than 1.0 are a conservative estimate of areas of consistent model response, as noted in Section 9.2.2 above.

There is relatively good agreement between the models for the lower latitude response, with larger range and less certain response at higher latitudes (Figure 9.10). For example, most models show a minimum of warming somewhere in the North Atlantic but the location is quite variable. There is a tendency for more warming (roughly a degree) in the tropical central and east Pacific than in the west, though this east-west difference in warming is generally less than a degree in the multi-model ensemble and is not evident with the contour interval in Figure 9.10 except in the B2 experiment in Figure 9.10e. This El Ninolike response is discussed further in Section 9.3.5.2.
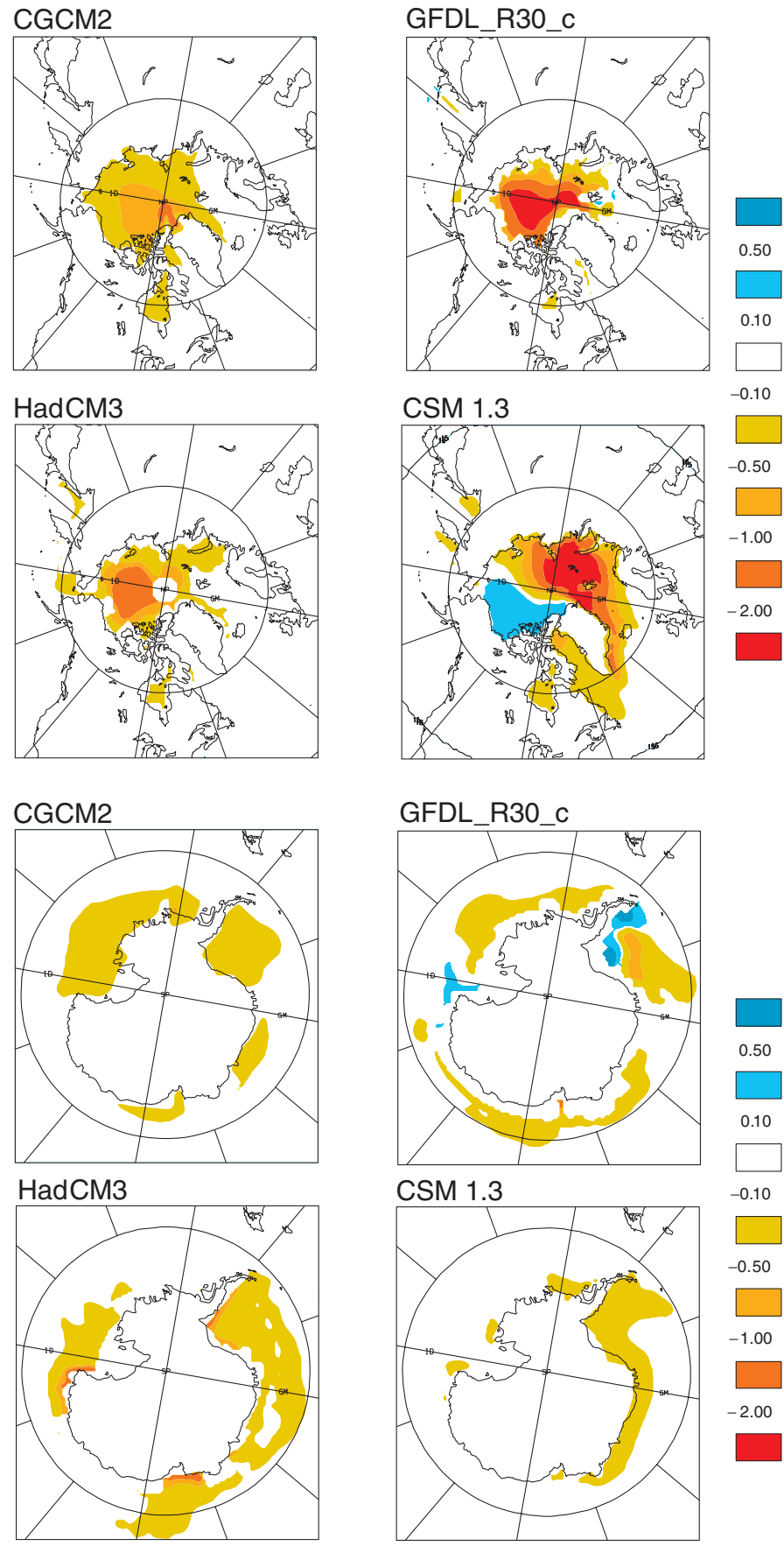

Figure 9.9: Change in annual mean sea-ice thickness between the periods 1971 to 1990 and 2041 to 2060 as simulated by four of the most recent coupled models. The upper panels show thickness changes in the Northern Hemisphere, the lower panels show changes in the Southern Hemisphere. All models were run with similar forcing scenarios: historical greenhouse gas and aerosol loading, then future forcing as per the IS92a scenario. The colour bar indicates thickness change in metres - negative values indicate a decrease in future ice thickness.

The biggest difference between the CMIP2 G (Figure 9.10a,b) and GS experiments (Figure 9.10c) is the regional moderating of the warming mainly over industrialised areas in GS where the negative forcing from sulphate aerosols is greatest at mid-21st century (note the regional changes 
Table 9.2: The pattern correlation of temperature and precipitation change for the years (2021 to 2050) relative to the years (1961 to 1990) for the simulations in the IPCC DDC. Above the diagonal: G experiments, below the diagonal: GS experiments. The diagonal is the correlation between $G$ and GS patterns from the same model.

\begin{tabular}{|c|c|c|c|c|c|c|c|c|c|}
\hline Temperature & $\begin{array}{c}\text { CGC } \\
\text { M1 }\end{array}$ & $\begin{array}{l}\text { CCSR/ } \\
\text { NIES }\end{array}$ & $\begin{array}{c}\text { CSIRO } \\
\text { Mk2 }\end{array}$ & $\begin{array}{c}\text { ECHAM3/ } \\
\text { LSG }\end{array}$ & $\begin{array}{c}\text { GFDL_ } \\
\text { R15_a }\end{array}$ & HadCM2 & HadCM3 & $\begin{array}{c}\text { ECHAM4/ } \\
\text { OPYC }\end{array}$ & DOE PCM \\
\hline CGCM1 & 0.96 & 0.74 & 0.65 & 0.47 & 0.65 & 0.72 & 0.67 & 0.65 & 0.31 \\
\hline CCSR/NIES & 0.75 & 0.97 & 0.77 & 0.45 & 0.72 & 0.77 & 0.73 & 0.80 & 0.49 \\
\hline CSIRO Mk2 & 0.61 & 0.71 & 0.96 & 0.40 & 0.75 & 0.72 & 0.67 & 0.75 & 0.63 \\
\hline ECHAM3/LSG & 0.58 & 0.50 & 0.44 & 0.46 & 0.40 & 0.53 & 0.60 & 0.53 & 0.35 \\
\hline GFDL_R15_a & 0.65 & 0.76 & 0.69 & 0.42 & 0.73 & 0.58 & 0.61 & 0.69 & 0.55 \\
\hline HadCM2 & 0.65 & 0.69 & 0.59 & 0.52 & 0.50 & 0.85 & 0.79 & 0.79 & 0.43 \\
\hline HadCM3 & 0.60 & 0.65 & 0.60 & 0.49 & 0.47 & 0.63 & 0.90 & 0.75 & 0.47 \\
\hline ЕСНАМ4/OPYC & 0.67 & 0.78 & 0.66 & 0.37 & 0.71 & 0.61 & 0.69 & 0.89 & 0.41 \\
\hline DOE PCM & 0.30 & 0.38 & 0.63 & 0.24 & 0.36 & 0.40 & 0.44 & 0.37 & 0.91 \\
\hline Precipitation & $\begin{array}{c}\text { CGC } \\
\text { M1 }\end{array}$ & $\begin{array}{c}\text { CCSR/ } \\
\text { NIES }\end{array}$ & $\begin{array}{c}\text { CSIRO } \\
\text { Mk2 }\end{array}$ & $\begin{array}{c}\text { ECHAM3/ } \\
\text { LSG }\end{array}$ & $\begin{array}{c}\text { GFDL_ } \\
\text { R15_a }\end{array}$ & HadCM2 & HadCM3 & $\begin{array}{c}\text { ECHAM4/ } \\
\text { OPYC }\end{array}$ & DOE PCM \\
\hline CGCM1 & 0.88 & 0.14 & 0.08 & 0.05 & 0.05 & 0.23 & -0.16 & -0.03 & 0.02 \\
\hline CCSR/NIES & 0.14 & 0.91 & 0.13 & 0.21 & 0.34 & 0.36 & 0.29 & 0.33 & 0.18 \\
\hline CSIRO Mk2 & 0.15 & 0.14 & 0.73 & 0.13 & 0.29 & 0.32 & 0.31 & 0.07 & 0.11 \\
\hline ECHAM3/LSG & 0.20 & 0.23 & 0.13 & 0.39 & 0.28 & 0.19 & 0.11 & 0.11 & 0.29 \\
\hline GFDL_R15_a & 0.18 & 0.20 & 0.28 & 0.14 & 0.41 & 0.28 & 0.20 & 0.22 & 0.21 \\
\hline HadCM2 & 0.34 & 0.34 & 0.23 & 0.37 & 0.24 & 0.73 & 0.19 & 0.24 & 0.17 \\
\hline HadCM3 & -0.20 & 0.06 & 0.31 & -0.05 & 0.11 & -0.01 & 0.81 & 0.25 & 0.09 \\
\hline ЕСНАМ4/OPYC & 0.13 & 0.30 & 0.09 & 0.07 & 0.04 & 0.23 & 0.20 & 0.79 & 0.01 \\
\hline DOE PCM & 0.02 & 0.08 & 0.12 & -0.09 & 0.06 & 0.13 & -0.06 & -0.07 & 0.43 \\
\hline
\end{tabular}

discussed in Chapter 10). This regional effect was noted in the SAR for only two models, but Figure 9.10c shows this is a consistent response across the greater number of more recent models. The GS experiments only include the direct effect of sulphate aerosols, but two model studies have included the direct and indirect effect of sulphate aerosols and show roughly the same pattern (Meehl et al., 1996; Roeckner et al., 1999). The simulations performed with and without the direct sulphate effect (GS and G, respectively) with the same model are more similar to each other than to the other models, indicating that the individual response characteristics of the various models are dominating the response pattern rather than differences in the forcing. With greater $\mathrm{CO}_{2}$ forcing, the simulated patterns are more highly correlated in the G simulations than in the GS simulations (Table 9.2, 26 of 36 possible model combinations for temperature, 22 of 36 for precipitation).

The SRES A2 and B2 integrations (Figure 9.10d,e) show a similar pattern of temperature change as the CMIP2 and G experiments. Since the positive radiative forcing from greenhouse gases overwhelms the sulphate aerosol forcing at the end of the 21st century in A2 and B2 compared to the GS experiments at mid-21st century, the patterns resemble more closely the G simulations in Figure 9.10a,b. The amplitude of the climate change patterns is weaker for the $\mathrm{B} 2$ than for the $\mathrm{A} 2$ simulations at the end of the 21st century (Figure 9.10d,e).

The relative change in the mean precipitation (Figure 9.11) for all models in all categories shows a general increase in the tropics (particularly the tropical oceans and parts of northern
Africa and south Asia) and the mid- and high latitudes, while the rainfall generally decreases in the sub-tropical belts. These changes are more evident for larger positive radiative forcing in the A2 and B2 scenario runs at the end of the 21st century (Figure 9.11d,e). This also applies to the areas of decrease that show a high inter-model variability and therefore little consistency among models, while in the tropics the change can exceed the variability of the signal by a factor of 2 . This is particularly evident over the central and eastern tropical Pacific where the El Niño-like surface temperature warming is associated with an eastward shift of positive precipitation anomalies. The A2 and B2 scenario experiments exhibit a relatively large increase in precipitation over the Sahara and Arabia, but with large intermodel variability. This is partly an artefact of using percentage change rather than absolute values, since in these regions the absolute precipitation amount is very small.

Other manifestations of the changes in precipitation are reported by Noda and Tokioka (1989), Murphy and Mitchell (1995) and Royer et al. (1998) who found an increase in the global mean convective rain rate in the $2 \times \mathrm{CO}_{2}$ climate compared with the $1 \times \mathrm{CO}_{2}$ climate. Results from another model (Brinkop, 2001; see also Cubasch et al., 1999) indicate a decrease in global mean convective precipitation. Essentially the results of Brinkop are consistent with Murphy and Mitchell, because in both transient climate simulations the strongest reduction in convective rain is found in the sub-tropics, and is most pronounced in the Southern Hemisphere. The increase in convective rain rate in the Northern Hemisphere is less strong in Brinkop compared to 




$$
\begin{array}{lllllllllll}
-1 & 0 & 1 & 2 & 3 & 4 & 5 & 6 & 8 & 10 & 12
\end{array}
$$

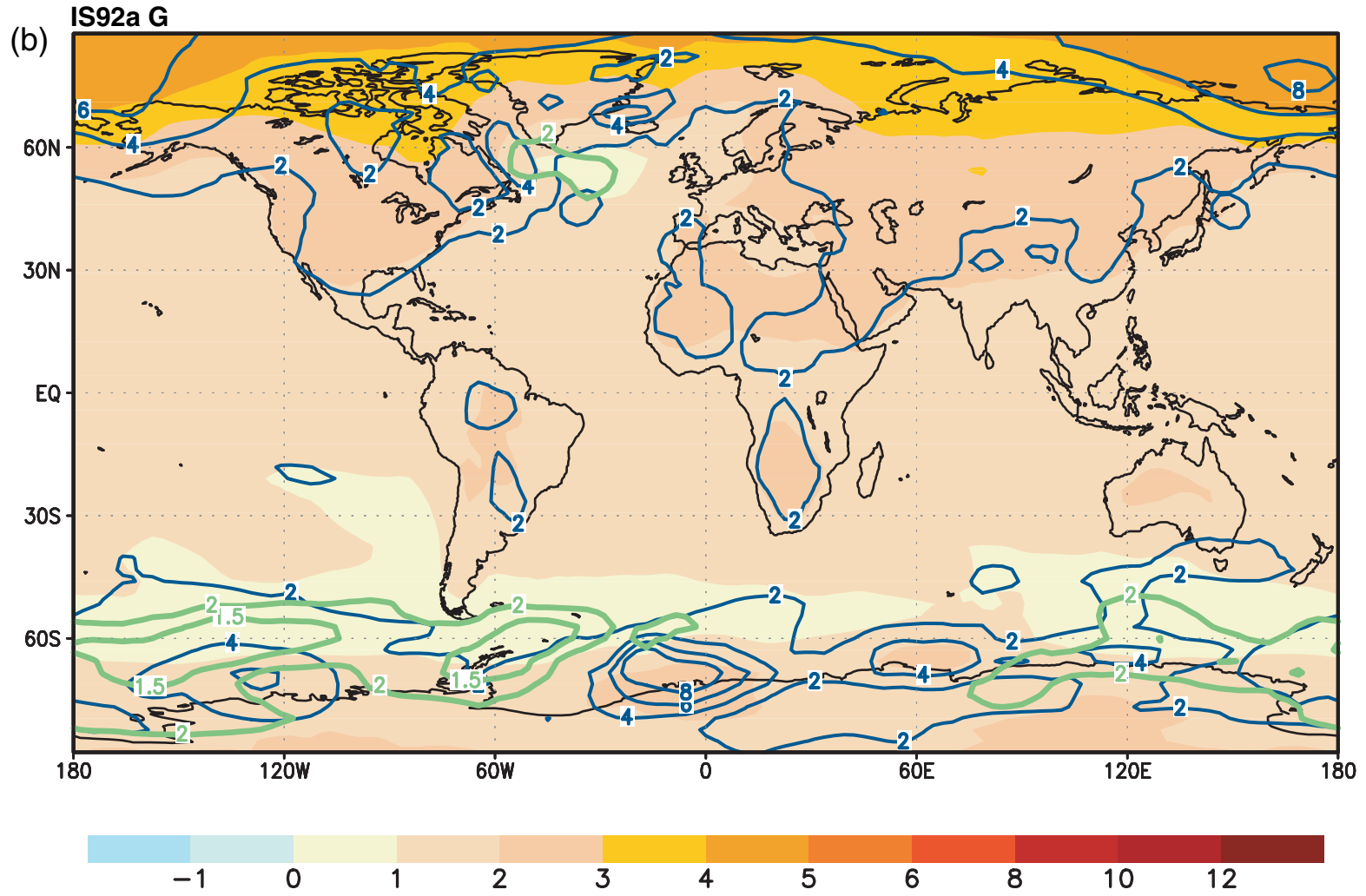

Figure 9.10: The multi-model ensemble annual mean change of the temperature (colour shading), its range (thin blue isolines) (Unit: ${ }^{\circ} \mathrm{C}$ ) and the multi-model mean change divided by the multi-model standard deviation (solid green isolines, absolute values) for (a) the CMIP2 scenarios at the time of $\mathrm{CO}_{2}$-doubling; (b) the IPCC-DDC scenario IS92a (G: greenhouse gases only) for the years 2021 to 2050 relative to the period 1961 to 1990; (c) the IPCC-DDC scenario IS92a (GS: greenhouse gases and sulphate aerosols) for the years 2021 to 2050 relative to the period 1961 to 1990; (d) the SRES scenario A2 and (e) the SRES scenario B2. Both SRES scenarios show the period 2071 to 2100 relative to the period 1961 to 1990. See text for scenario definitions and description of analysis technique. In (b) and (d) the ratio mean change/standard deviation is increasing towards the low latitudes as well as in (a), (c) and (e), while the high latitudes around Antarctica show a minimum. 

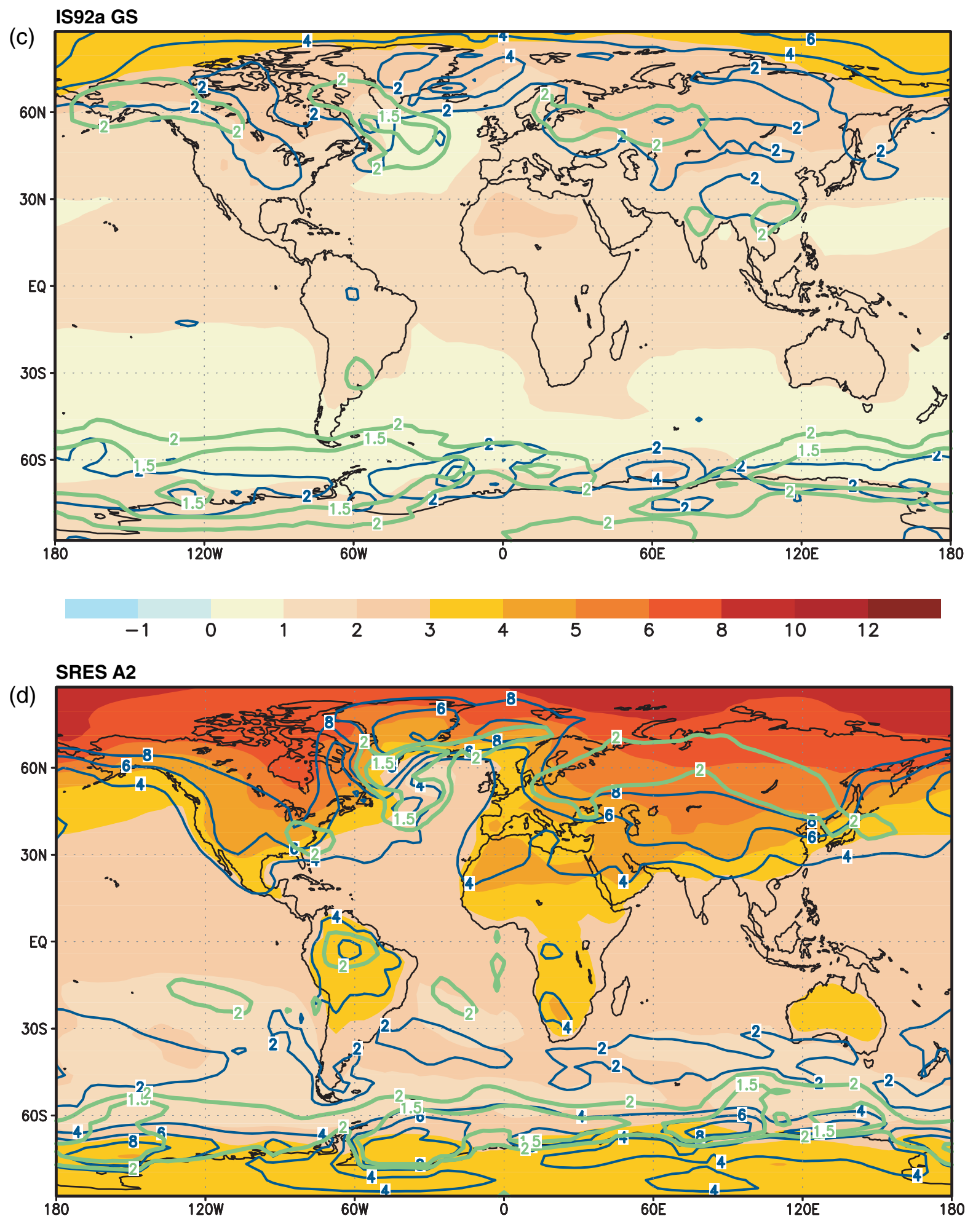

$\begin{array}{lllllllllll}-1 & 0 & 1 & 2 & 3 & 4 & 5 & 6 & 8 & 10 & 12\end{array}$

Figure 9.10: (c) and (d) Caption at Figure 9.10 (a). 


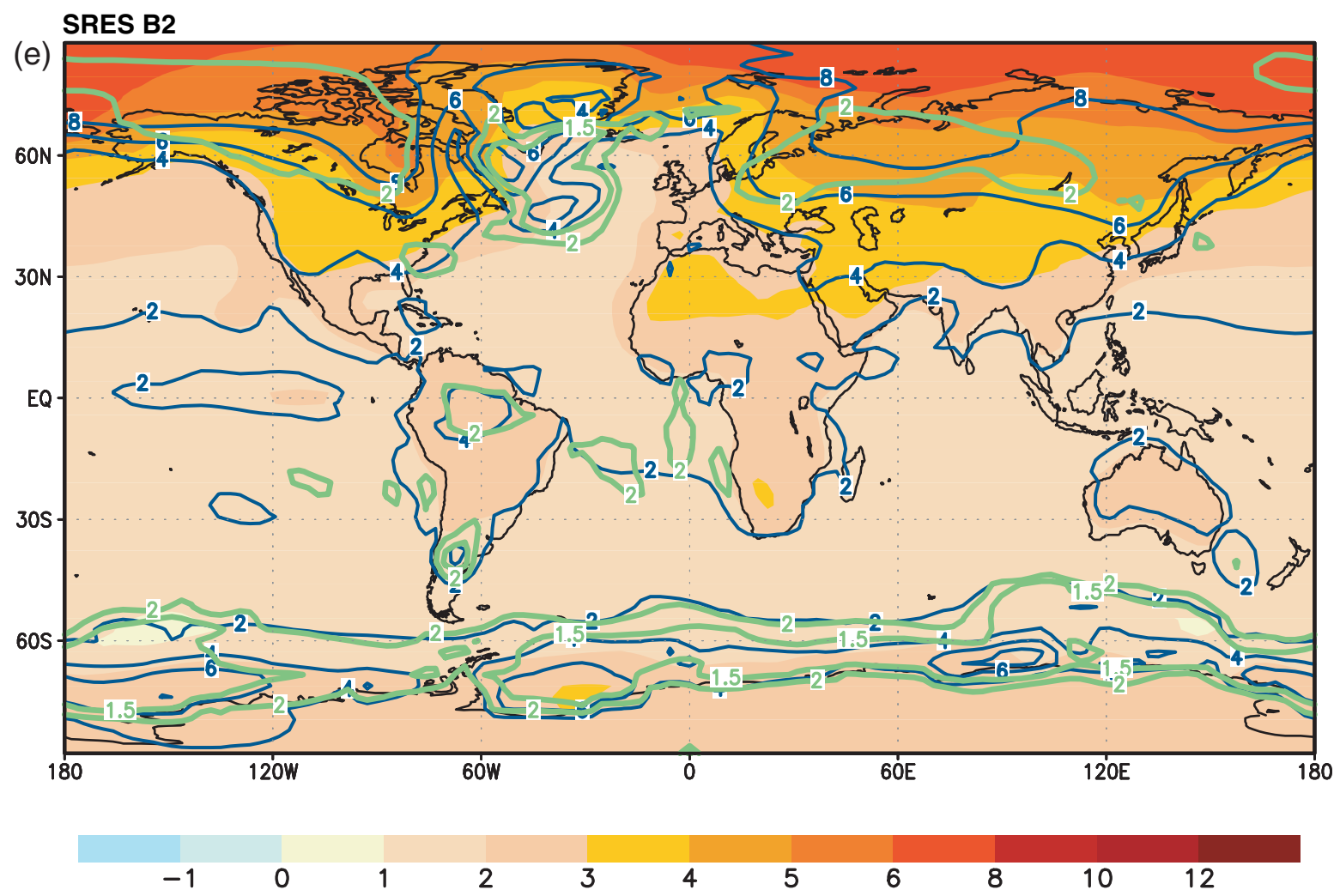

Figure 9.10: (e) Caption at Figure 9.10 (a).

Murphy and Mitchell, resulting in the decrease in global mean convective rain rate. In both models the origin of the decrease in convective precipitation is an increase in stability in the troposphere in the warmer climate. In accordance with the reduction in convective precipitation, Brinkop analysed a strong decrease ( $11 \%$ for JJA and $7.5 \%$ for DJF) of the global mean frequency of deep convection in the warmer climate. However, the frequency of shallow convection slightly increases.

The most consistent feature in the ensemble mean sea level pressure difference (Figure 9.12) is a decrease in the sea level pressure at high latitudes and an increase at mid-latitudes. In studies of the the Southern Hemisphere, this is related to a combination of changes in surface and mid-tropospheric temperature gradients (Räisänen, 1997; Fyfe et al., 1999; Kushner et al., 2001). Over wide regions of the Southern Hemisphere and Northern Hemisphere high latitudes, the ensemble mean signal generally exceeds the ensemble standard deviation indicating a consistent response across the models. For the A2 and B2 scenarios this is also found. Additionally a lowering of pressure can be found over the Sahara, probably due to thermal effects. The lowering of pressure is consistent across the A2 and B2 simulations.

\subsubsection{Summary}

First we note results assessed here that reconfirm results from the SAR:

- As the climate warms, Northern Hemisphere snow cover and sea-ice extent decrease. The globally averaged precipitation increases.
- As the radiative forcing of the climate system changes, the land warms faster than the ocean. The cooling effect of tropospheric aerosols moderates warming both globally and locally.

- The surface air temperature increase is smaller in the North Atlantic and circumpolar Southern Ocean regions.

- Most tropical areas, particularly over ocean, have increased precipitation, with decreases in most of the sub-tropics, and relatively smaller precipitation increases in high latitudes.

- The signal to noise ratio (from the multi-model ensemble) is greater for surface air temperature than for precipitation.

A second category of results assessed here are those that are new since the SAR:

- There are many more model projections for a given scenario, and more scenarios. The greater number of model simulations allows us to better quantify patterns of climate change for a given forcing and develop a measure of consistency among the models.

- Including the direct effect of sulphate aerosols according to an IS92a type estimate reduces global mean mid-21st century warming. The indirect effect, not included in most AOGCM experiments to date, is acknowledged to be uncertain, as discussed in Chapter 6.

- The geographic details of various forcing patterns are less important than differences among the models' responses for the scenarios considered here. This is the case for the global mean as well as for patterns of climate response. Thus, the choice of model and the choice of scenario are both important. 

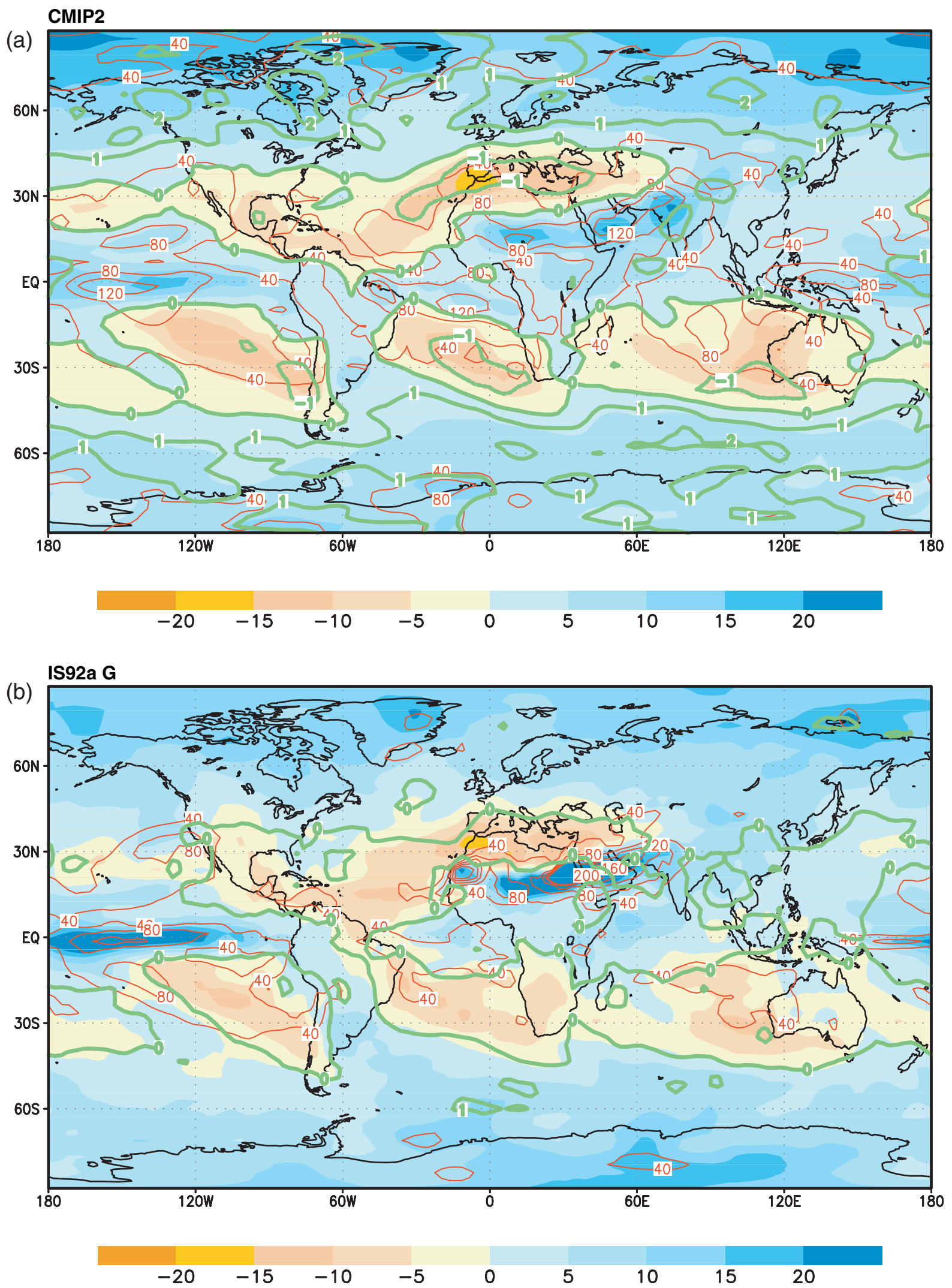

Figure 9.11: The multi-model ensemble annual mean change of the precipitation (colour shading), its range (thin red isolines) (Unit: \%) and the multi-model mean change divided by the multi-model standard deviation (solid green isolines, absolute values) for (a) the CMIP2 scenarios at the time of $\mathrm{CO}_{2}$-doubling; (b) the IPCC-DDC scenario IS92a (G: greenhouse gases only) for the years 2021 to 2050 relative to the period 1961 to 1990; (c) the IPCC-DDC scenario IS92a (GS: greenhouse gases and sulphate aerosols) for the years 2021 to 2050 relative to the period 1961 to 1990; (d) the SRES scenario A2; and (e) the SRES scenario B2. Both SRES-scenarios show the period 2071 to 2100 relative to the period 1961 to 1990. See text for scenario definitions and description of analysis technique. 

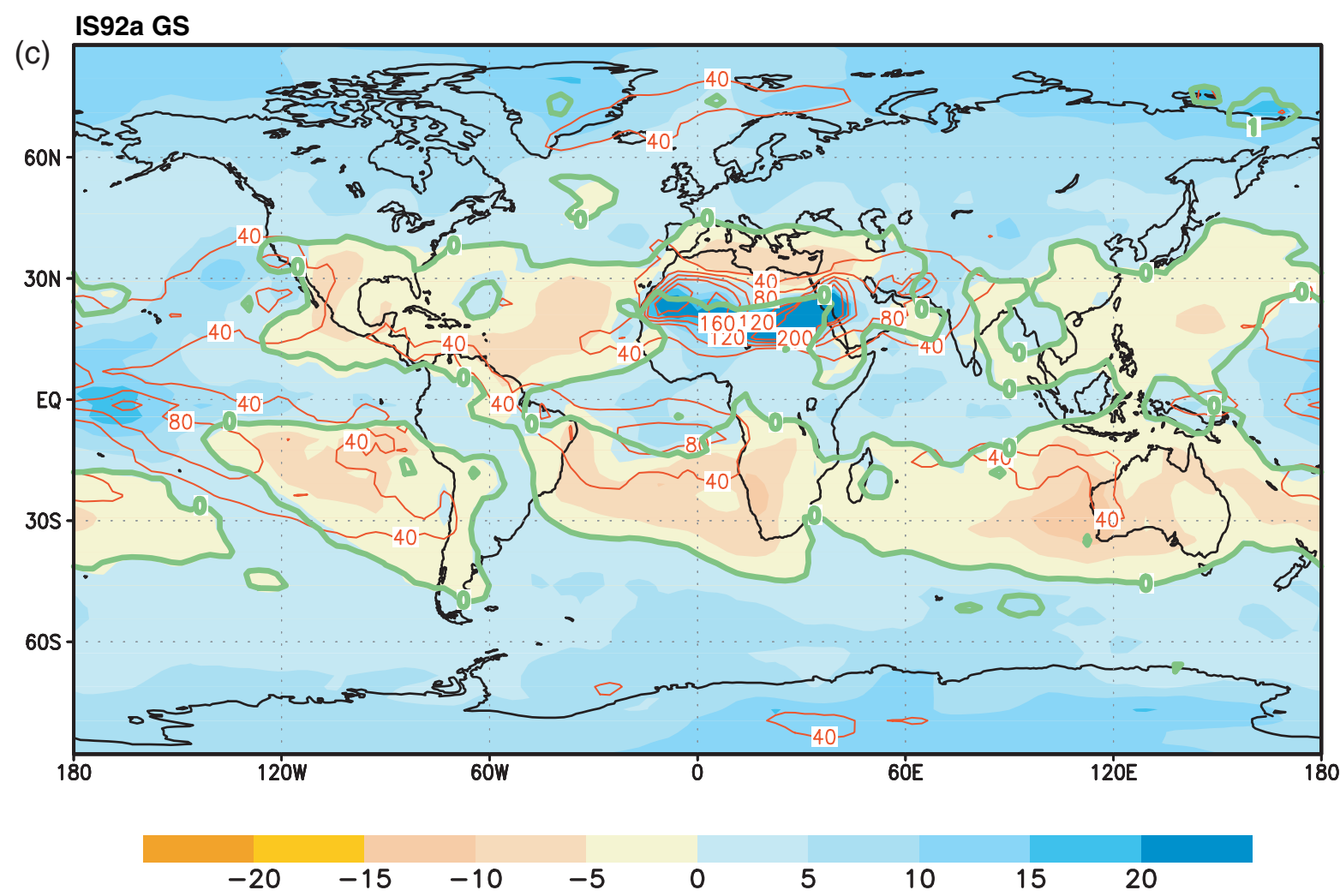

\section{SRES A2}

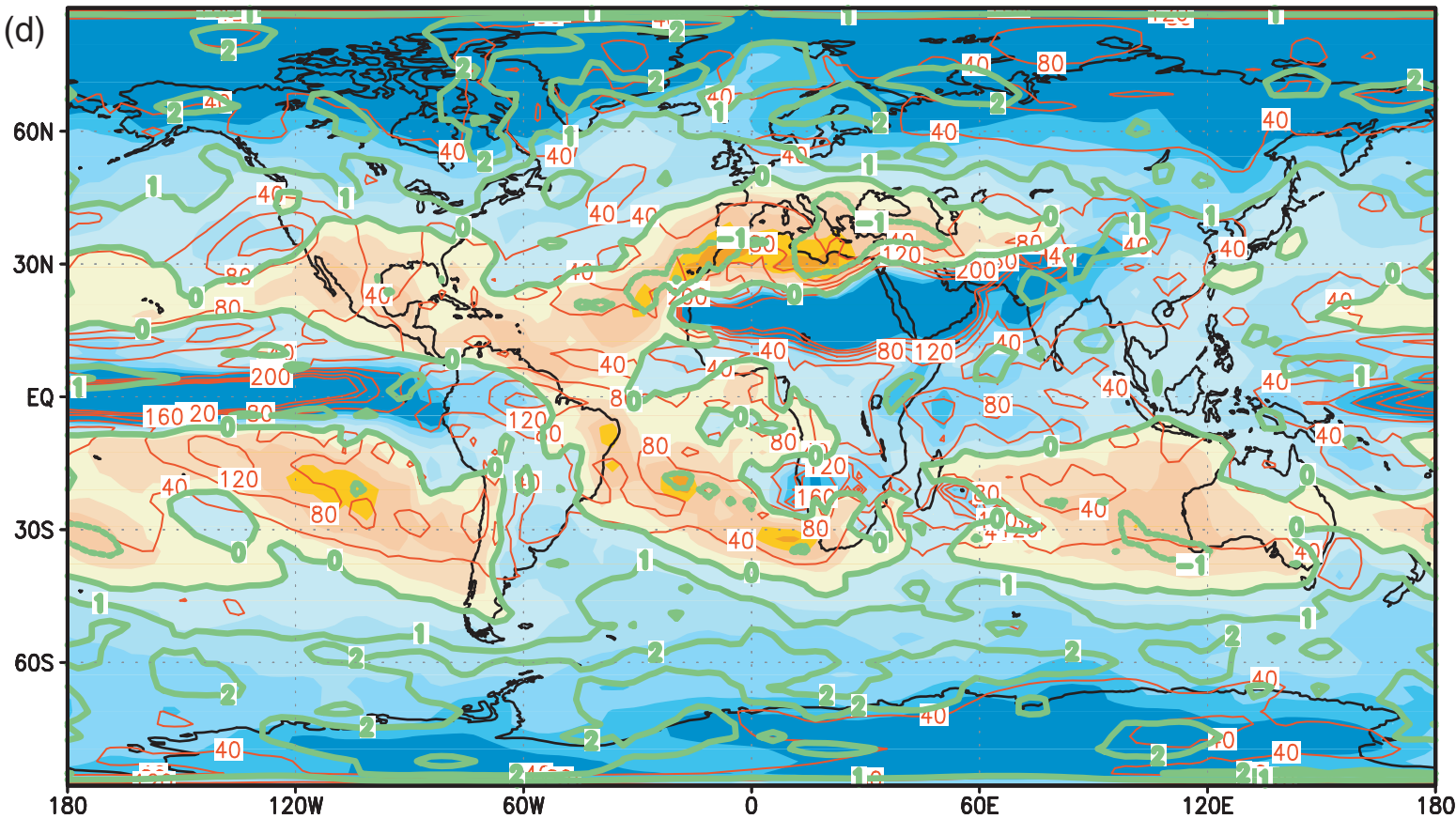

$\begin{array}{lllllllll}-20 & -15 & -10 & -5 & 0 & 5 & 10 & 15 & 20\end{array}$

Figure 9.11: (c) and (d) Caption at Figure 9.11 (a). 


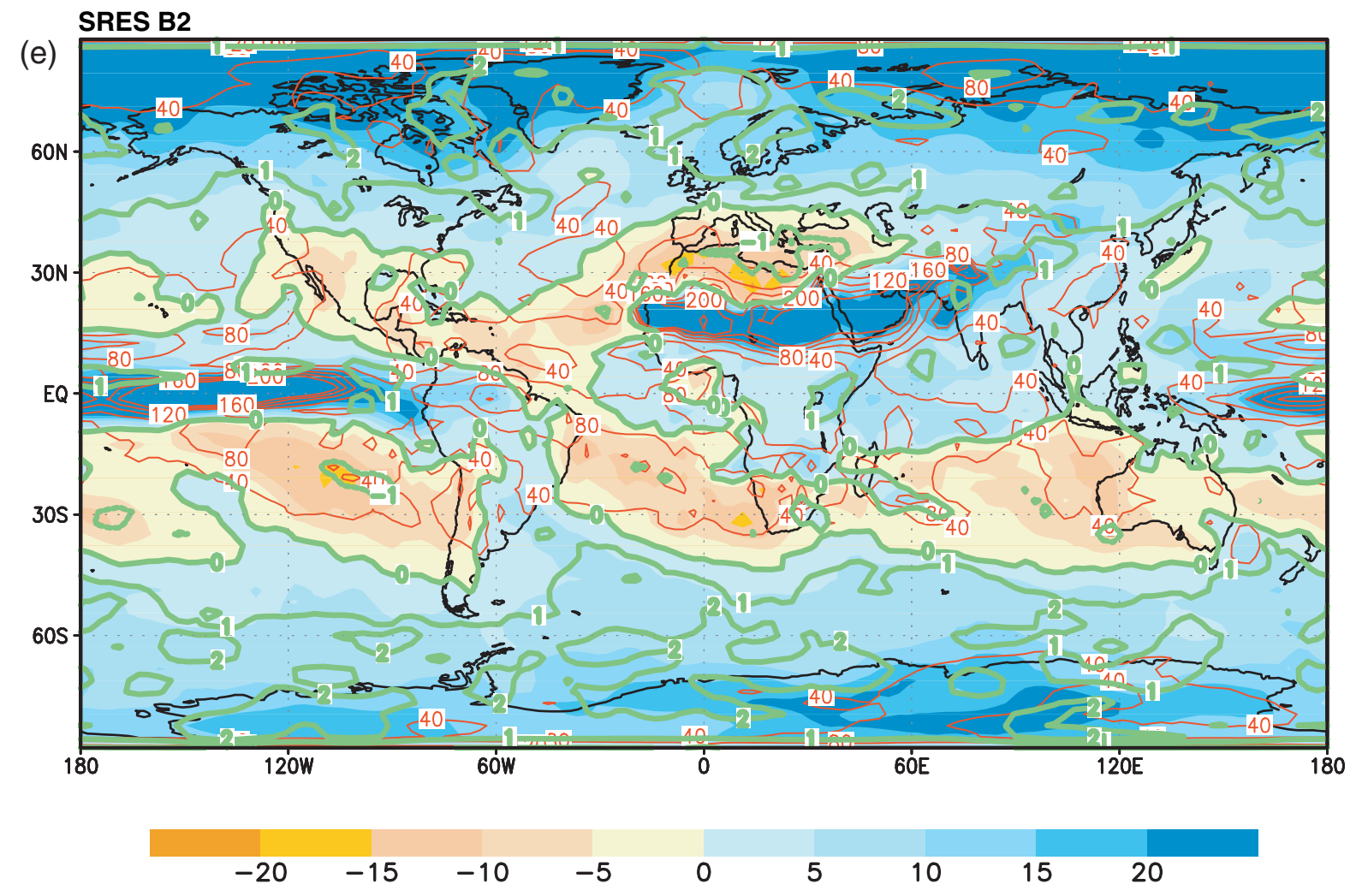

Figure 9.11: (e) Caption at Figure 9.11 (a).

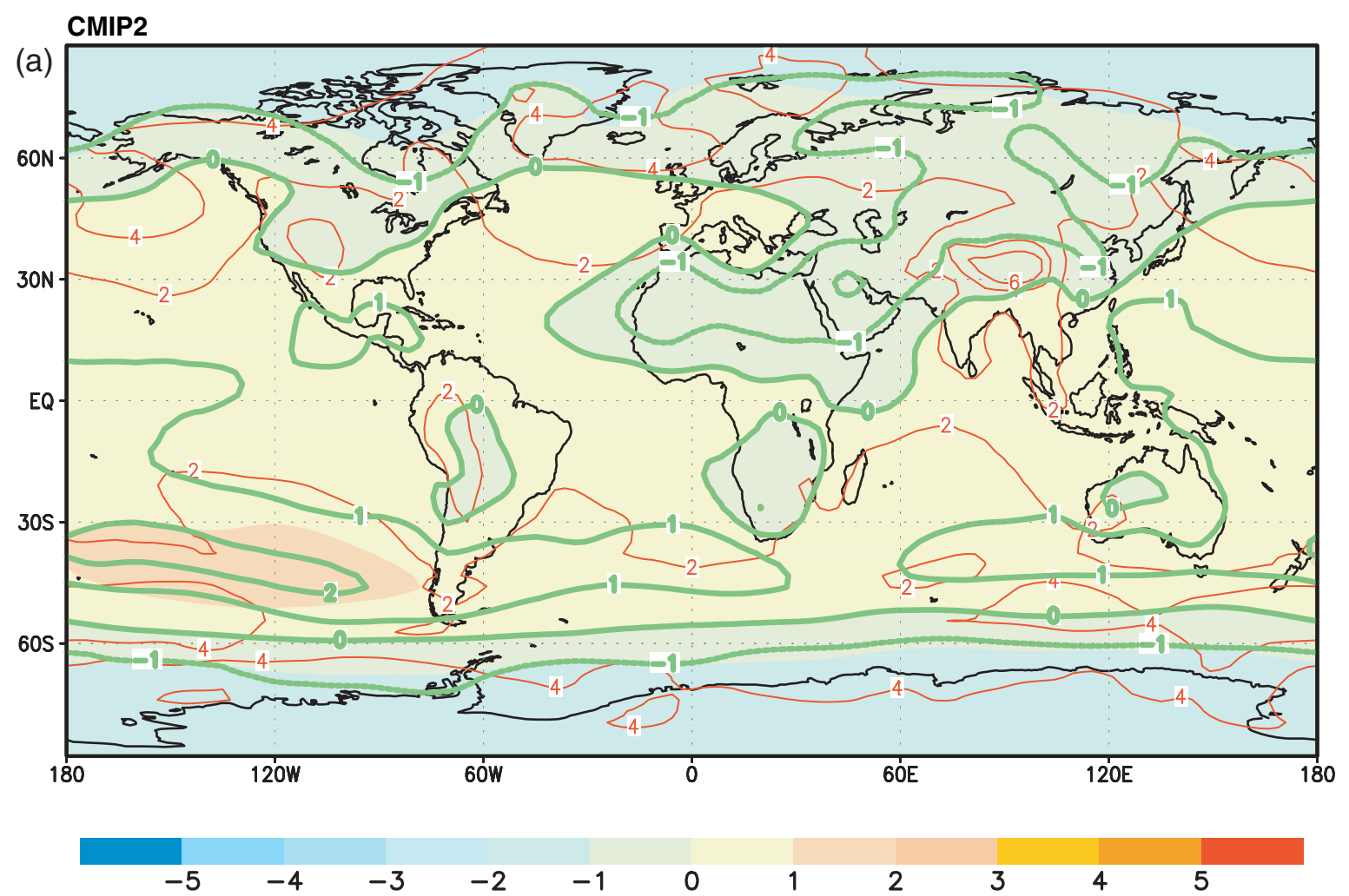

Figure 9.12: The multi-model ensemble annual mean change of the sea level pressure (colour shading), its range (thin red isolines) (Unit: hPa) and the multi-model mean change divided by the multi-model standard deviation (solid green isolines, absolute values) for (a) the CMIP2 scenarios at the time of $\mathrm{CO}_{2}$-doubling; (b) the IPCC-DDC scenario IS92a (G: greenhouse gases only) for the years 2021 to 2050 relative to the period 1961 to 1990; (c) the IPCC-DDC scenario IS92a (GS: greenhouse gases and sulphate aerosols) for the years 2021 to 2050 relative to the period 1961 to 1990; (d) the SRES scenario A2 and (e) the SRES scenario B2. Both SRES-scenarios show the period 2071 to 2100 relative to the period 1961 to 1990 . See text for scenario definitions and description of analysis technique. 

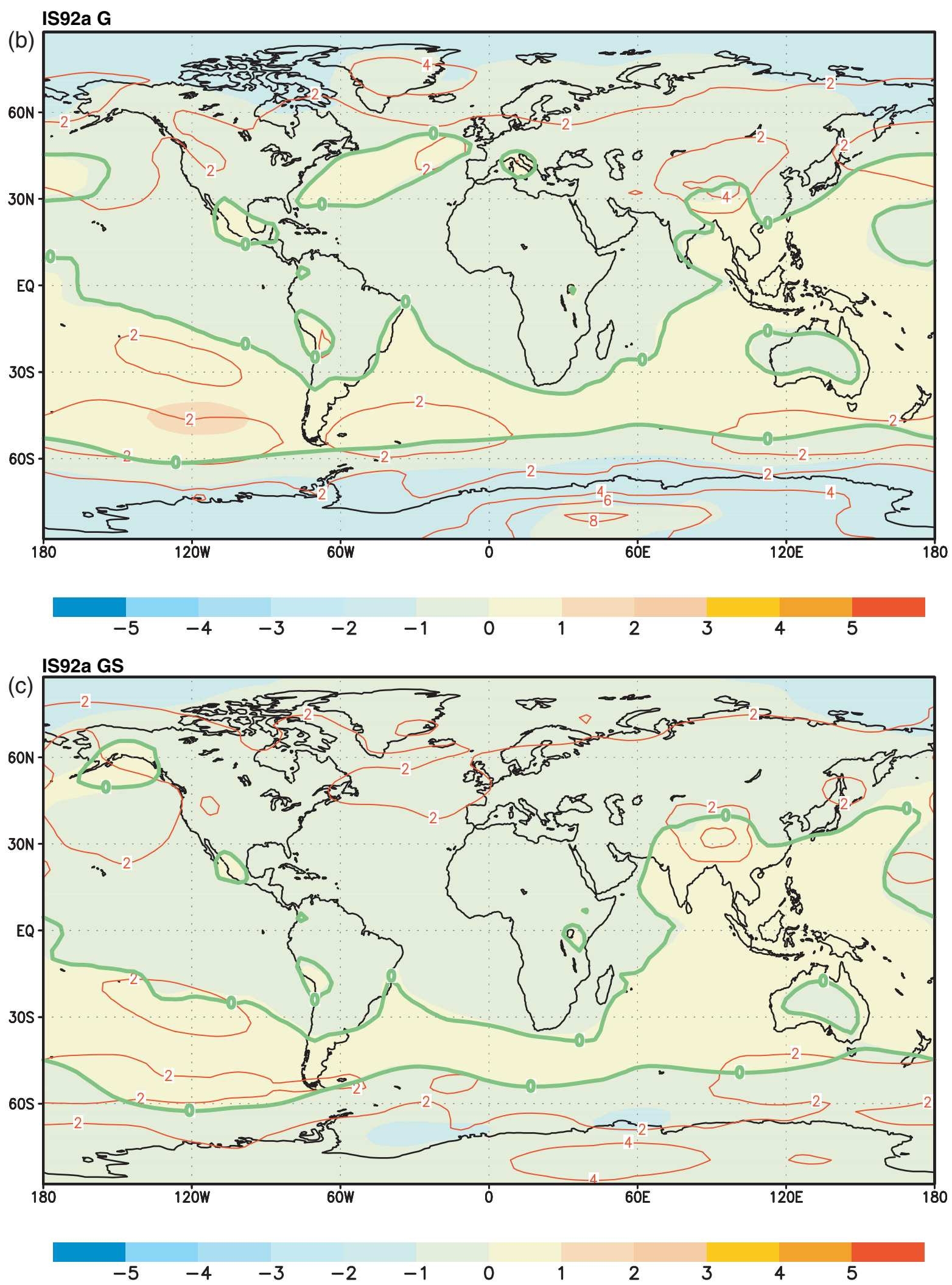

Figure 9.12: (b) and (c) Caption at Figure 9.12 (a). 

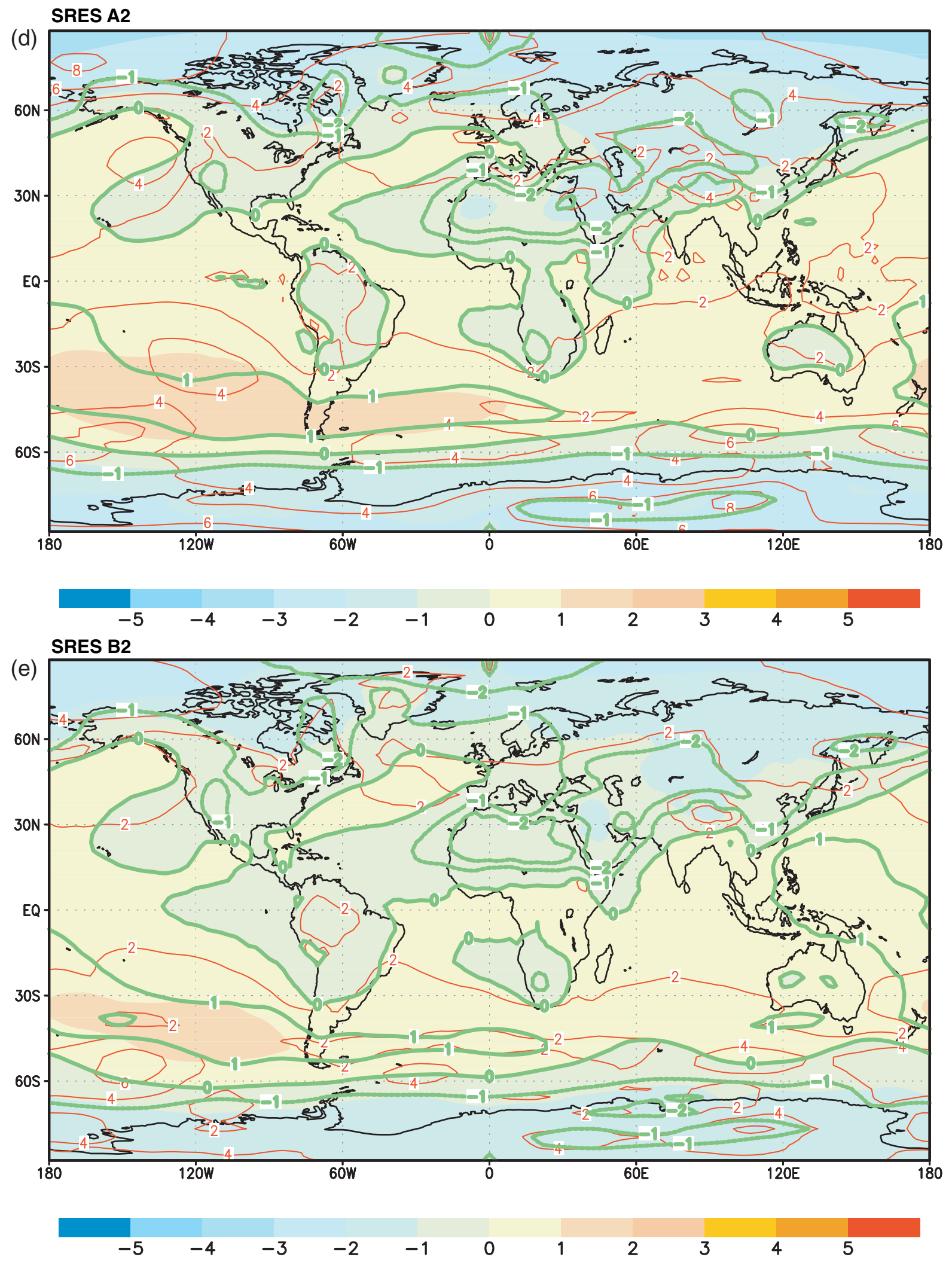

Figure 9.12: (d) and (e) Caption at Figure 9.12 (a). 


\subsubsection{Range of Temperature Response to SRES Emission Scenarios}

This section investigates the range of future global mean temperature changes resulting from the thirty-five final SRES emissions scenarios with complete greenhouse gas emissions (Nakićenović $e t$ al., 2000). This range is compared to the expected range of uncertainty due to the differences in the response of several AOGCMs. Forcing uncertainties are not considered in these calculations. As well as envelope results that incorporate all the SRES scenarios, six specific SRES scenarios are considered. These are the four illustrative marker scenarios A1B, A2, B1 and B2 and two further illustrative scenarios from the A1 family representing different energy technology options; A1FI and A1T (see Section 9.3.1.3 and Box 9.1). For comparison, results are also shown for some of the IS92 scenarios. As discussed in Section 9.3.1.3 some AOGCMs have run experiments with some or all of the four draft marker scenarios. In order to investigate the temperature change implications of the full range of the final SRES scenarios, a simple climate model is used as a tool to simulate the AOGCM results (Wigley and Raper, 1992; Raper et al., 1996, 2001a). The tuning of the simple model to emulate the different AOGCM results is described in Appendix 9.1. The original SRES MiniCAM (Mini Climate Assessment Model from the Pacific Northwest National Laboratory, USA) scenarios did not contain emissions for the reactive gases CO, NMVOCs, and $\mathrm{NO}_{\mathrm{x}}$ (Nakićenović et al., 2000). To facilitate the calculations, the MiniCAM modelling team provided emissions paths for these gases.

For the six illustrative SRES scenarios, anthropogenic emissions are shown for $\mathrm{CO}_{2}$ in Chapter 3, Figure 3.12, tabulated for $\mathrm{CH}_{4}$ and $\mathrm{N}_{2} \mathrm{O}$ in Appendix II and shown in Nakićenović et al. (2000), and shown for $\mathrm{SO}_{2}$ in Chapter 5, Figure 5.13. It is evident that these scenarios encompass a wide range of emissions. Note in particular the much lower future sulphur dioxide emissions for the six SRES scenarios compared with the IS92a scenario.

The calculation of radiative forcing from the SRES emission scenarios for the temperature projections presented here follows closely that described in Chapters 3, 4, 5 and 6, with some exceptions as described below. Further details of the forcing for the collective procedures (MAGICC model) are given by Wigley (2000). Atmospheric concentrations of the greenhouse gases are calculated from the emissions using gas cycle models. For $\mathrm{CO}_{2}$, the model of Wigley (1993) is used and as described therein, the $\mathrm{CO}_{2}$ fertilisation factor is adjusted to give a balanced 1980s mean budget. To be consistent with Chapter 3, climate feedbacks are included and the model has been tuned to give results that are similar to those of the Bern-CC and ISAM models for a climate sensitivity of $2.5^{\circ} \mathrm{C}$ (Chapter 3, Figure 3.12). The strength of the climate feedbacks on the carbon cycle are very uncertain, but models show they are in the direction of greater temperature change giving greater atmospheric $\mathrm{CO}_{2}$ concentration. The climate feedbacks in the Bern-CC model are greater than those of the ISAM model and the feedback strength used here is about half as big as that in the ISAM model. The gas cycle models for $\mathrm{CH}_{4}$ and $\mathrm{N}_{2} \mathrm{O}$ and the other trace gases are identical to those used in Chapter 4. The concentrations for the main greenhouse gases for the six SRES scenarios are shown in Chapter 4, Figure 4.14.
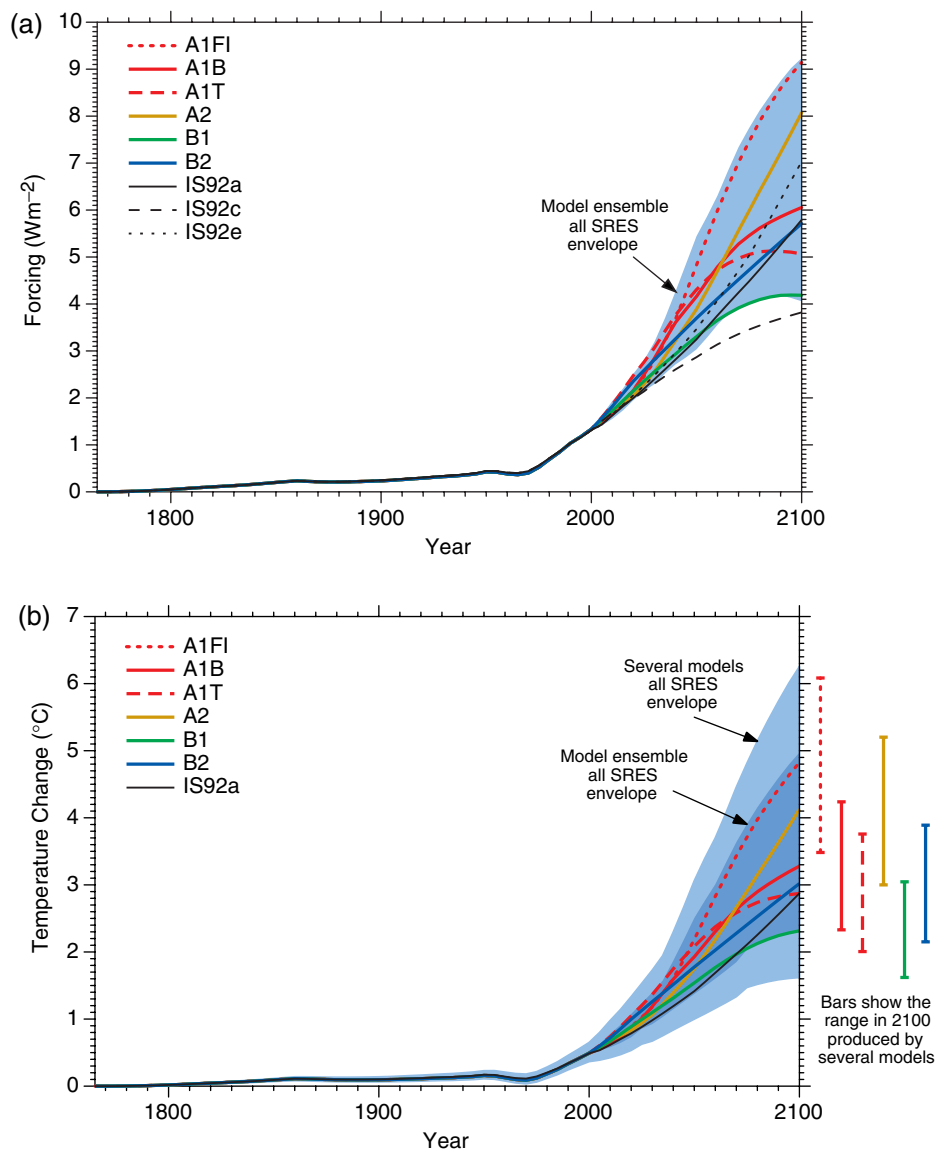

Figure 9.13: Simple model results. (a) Estimated historical anthropogenic radiative forcing followed by radiative forcing for the four illustrative SRES marker scenarios and for two additional scenarios from the A1 family illustrating different energy technology options. The blue shading shows the envelope of forcing that encompasses the full set of thirty-five SRES scenarios. The method of calculation closely follows Chapter 6 except where explained in the text. The values are based on the radiative forcing for a doubling of $\mathrm{CO}_{2}$ from seven AOGCMs as given in Appendix 9.1, Table 9.A1. The IS92a, IS92c and IS92e forcing is also shown following the same method of calculation. (b) Historical anthropogenic global mean temperature change and future changes for the six illustrative SRES scenarios using a simple climate model tuned to seven AOGCMs. Also for comparison, following the same method, results are shown for IS92a. The dark blue shading represents the envelope of the full set of thirty-five SRES scenarios using the simple model ensemble mean results. The light blue envelope is based on the GFDL_R15_a and DOE PCM parameter settings. The bars show the range of simple model results in 2100 for the seven AOGCM model tunings.

Except for the treatment of organic carbon (OC), black carbon (BC) and indirect aerosol forcing, the method of calculation for the radiative forcing follows closely that described in Chapter 6 and includes tropospheric ozone, halocarbons, and stratospheric ozone. For OC and BC this report's best estimate forcing values for the present day given in Chapter 6, Table 6.11 are used. As pointed out in Chapter 5, past and future emissions of $\mathrm{OC}$ and $\mathrm{BC}$ are uncertain. Here fossil $\mathrm{OC}$ and $\mathrm{BC}$ direct aerosol 


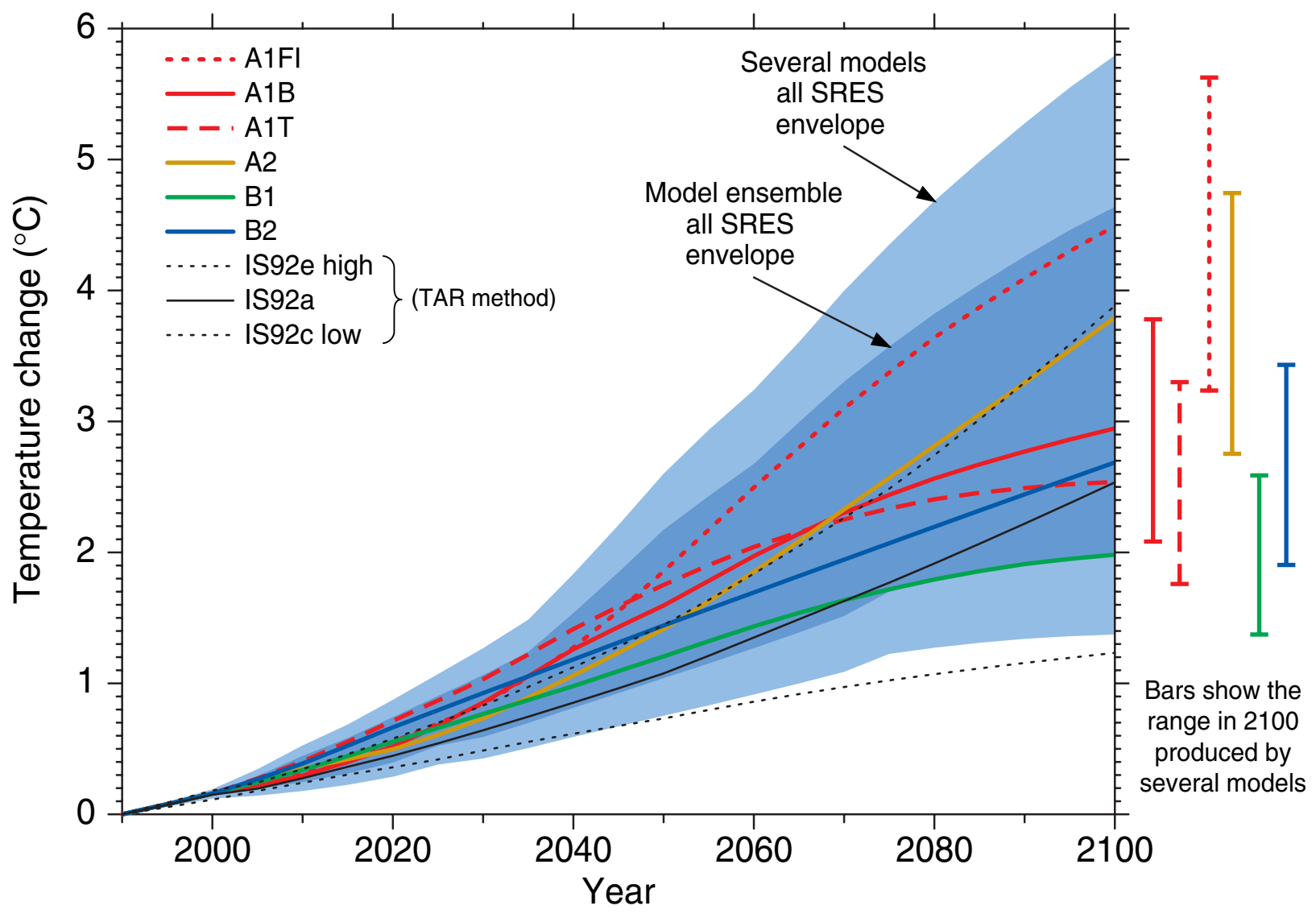

Figure 9.14: As for Figure 9.13b but results are relative to 1990 and shown for 1990 to 2100.

forcings are considered together and are scaled linearly with $\mathrm{SO}_{2}$ emissions. Biomass burning $\mathrm{OC}$ and $\mathrm{BC}$ aerosol direct forcings are both scaled with gross deforestation. First (cloud albedo) indirect sulphate aerosol forcing components are included and scaled non-linearly with $\mathrm{SO}_{2}$ emissions as derived by Wigley (1991). A present day indirect sulphate aerosol forcing of -0.8 $\mathrm{Wm}^{-2}$ is assumed. This is the same value as that employed in the SAR. It is well within the range of values recommended by Chapter 6, and is also consistent with that deduced from model simulations and the observed temperature record (Chapter 12).

Estimated total historical anthropogenic radiative forcing from 1765 to 1990 followed by forcing resulting from the six illustrative SRES scenarios are both shown in Figure 9.13a. It is evident that the six SRES scenarios considered cover nearly the full range of forcing that results from the full set of SRES scenarios. The latter is shown on figure 9.13a as an envelope since the forcing resulting from individual scenarios cross with time. For comparison, radiative forcing is also shown for the IS92a, IS92c and IS92e scenarios. It is evident that the range in forcing for the new SRES scenarios is wider and higher than in the IS92 scenarios. The range is wider due to more variation in emissions of non- $\mathrm{CO}_{2}$ greenhouse gases. The shift to higher forcing is mainly due to the reduced future sulphur dioxide emissions of the SRES scenarios compared to the IS92 scenarios. Secondary factors include generally greater tropospheric ozone forcing, the inclusion of climate feedbacks in the carbon cycle and slightly larger cumulative carbon emissions featured in some SRES scenarios.

Figure 9.13b shows the simple climate model simulations representing AOGCM-calibrated global mean temperature change results for the six illustrative SRES scenarios and for the full SRES scenario envelopes. The individual scenario timeseries and inner envelope (darker shading) are the average results obtained from simulating the results of seven AOGCMs, denoted "ensemble". The average of the effective climate sensitivity of these AOGCMs is $2.8^{\circ} \mathrm{C}$ (see Appendix 9.1). The range of global mean temperature change from 1990 to 2100 given by the six illustrative scenarios for the ensemble is 2.0 to $4.5^{\circ} \mathrm{C}$ (see Figure 9.14). The range for the six illustrative scenarios encompassing the results calibrated to the DOE PCM and GFDL_R15_a AOGCM parameter settings is 1.4 to $5.6^{\circ} \mathrm{C}$. These two AOGCMs have effective climate sensitivities of 1.7 and $4.2^{\circ} \mathrm{C}$, respectively (see Table 9.1). The range for these two parameter settings for the full set of SRES scenarios is 1.4 to $5.8^{\circ} \mathrm{C}$. Note that this is not the extreme range of possibilities, for two reasons. First, forcing uncertainties have not been considered. Second, some AOGCMs have effective climate sensitivities outside the range considered (see Table 9.1). For example, inclusion of the simple model's representation of the CCSR/NIES2 AOGCM would increase the high end of the range by several degrees $\mathrm{C}$. 
(a) Temperature change from 1990 to 2030

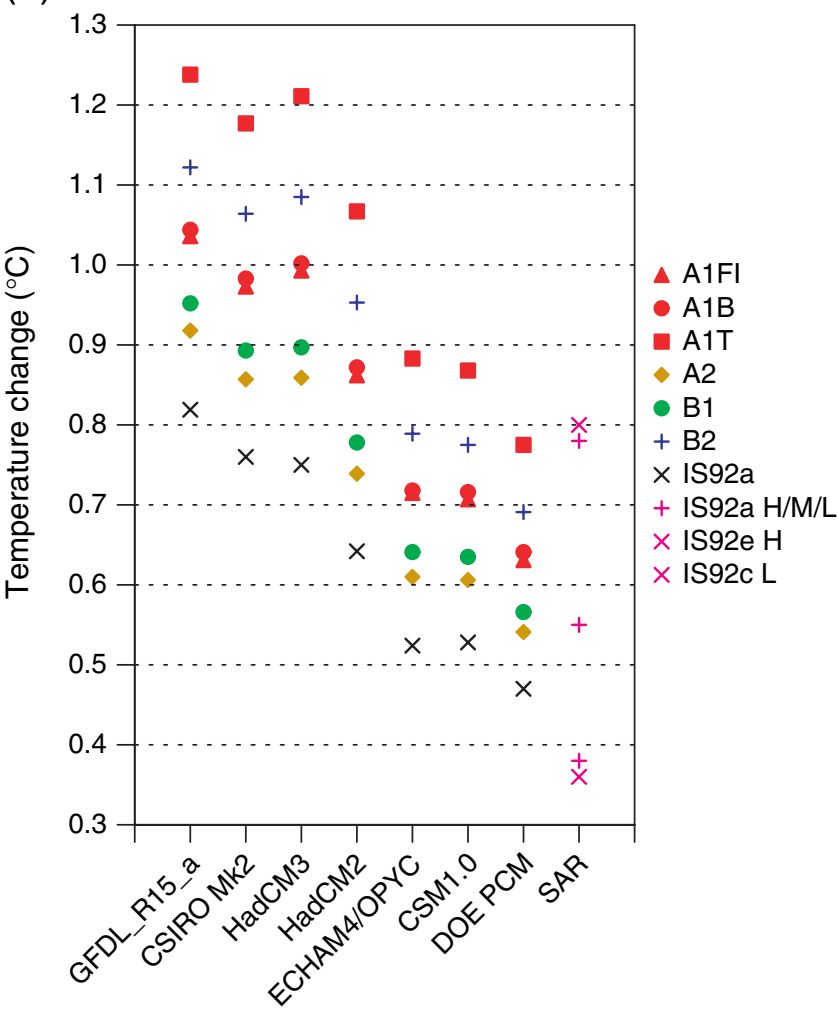

(b) Temperature change from 1990 to 2100

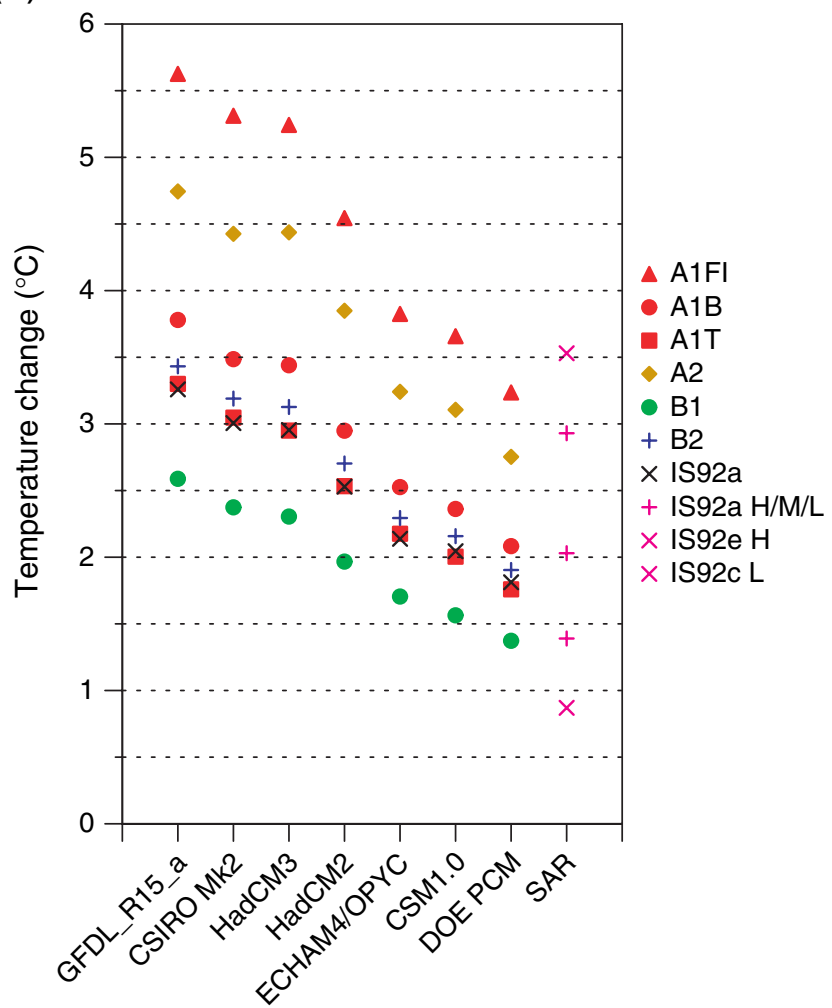

Figure 9.15: Simple model results: Temperature changes from (a) 1990 to 2030 and from (b) 1990 to 2100 for the six illustrative SRES scenarios and IS92a. The bottom axis indicates the AOGCM to which the simple model is tuned. For comparison results are also shown for the SAR version of the simple climate model using SAR forcing with some of the IS92 scenarios (see Kattenberg et al., 1996). IS92a H/M/L refers to the IS92a scenario with climate sensitivity of $1.5,2.5$ and $4.5^{\circ} \mathrm{C}$ respectively. Also shown are the IS92e scenario with a sensitivity of $4.5^{\circ} \mathrm{C}$ and the IS92c scenario with a sensitivity of $1.5^{\circ} \mathrm{C}$.

Since the AOGCM SRES results discussed in Section 9.3.1.3 are based on the draft marker SRES scenarios, it is important to note differences that would result from the use of the final SRES scenarios. Based on a comparison using the simple climate model, the final scenarios for the three markers A1B, A2 and $\mathrm{B} 2$ give temperature changes that are slightly smaller than those of the draft scenarios (Smith et al., 2001). The main difference is a change in the standardised values for 1990 through 2000, which are common to all these scenarios. This results in higher forcing early in the period. There are further small differences in net forcing, but these decrease until, by 2100 , differences in temperature change in the two versions of these scenarios are only 1 to $2 \%$. For the B1 scenario, however, temperature changes are significantly lower in the final version. The difference is almost $20 \%$ in 2100 , as a result of generally lower emissions across the whole range of greenhouse gases.

Temperature change results from the simple climate model tuned to individual AOGCMs using the six illustrative SRES scenarios are shown in Figure 9.15. For comparison, analogous results are shown for the IS92a scenario. For direct comparison with the SAR, results are also shown for some of the IS92 scenarios using the SAR forcing and the SAR version of the simple climate model (Kattenberg et al., 1996). The results give rise to conclusions similar to those of Wigley (1999) and Smith et al. (2001), which were drawn from sensitivity studies using the SAR version of the simple climate model. First, note that the range of temperature change for the SRES scenarios is shifted higher than the range for the IS92 scenarios, primarily because of the higher forcing as described above.

A second feature of the illustrative SRES scenarios is that their relative ranking in terms of global mean temperature changes varies with time (Wigley, 1999; Smith et al. 2001). The temperature-change values of the scenarios cross in about midcentury because of links between the emissions of different gases. In particular, for scenarios with higher fossil fuel use, and therefore carbon dioxide emissions (for example A2), sulphur dioxide emissions are also higher. In the near term (to around 2050) the cooling effect of higher sulphur dioxide emissions more than offsets the warming caused by increased emissions of greenhouse gases in scenarios such as A2. The effect of the high sulphur dioxide emissions in the IS92a scenario is similar. It causes IS92a to give rise to a lower 2030 temperature than any of the specific SRES scenarios considered (Figure 9.15a). The opposite effect is seen for scenarios B1 and B2, which have lower fossil fuel emissions, but also lower sulphur dioxide emissions. This leads to a larger near-term warming. In the longer term, however, the level of emissions of long-lived greenhouse gases such as carbon dioxide and nitrous oxide becomes the dominant 
determinant of the resulting global mean temperature changes. For example, by the latter part of the 21st century, the higher emissions of greenhouse gases in scenario A2 result in larger climate changes than in the other three marker scenarios (A1B, B1 and B2) even though this scenario also has higher sulphur dioxide emissions.

Considering the six illustrative scenarios, the bars on the right-hand side of Figure 9.14 show that scenarios A1FI and B1 alone, define the top and bottom of the range of projected temperature changes, respectively. Towards the middle of the range the scenario bars overlap, indicating that most of the projections fall within this region. In the corresponding sea level rise figure, because of the greater intertia in the ocean response, there is a greater overlap in the projected response to the various scenarios (see Chapter 11, Figure 11.12). In addition, the sea level range for a given scenario is broadened by inclusion of uncertainty in land ice estimates.

By 2100, the differences in the surface air temperature response across the group of climate models forced with a given scenario is as large as the range obtained by a single model forced with the different SRES scenarios (Figure 9.15). Given the quasilinear nature of the simple model, projections which go outside the range as yet explored by AOGCMs must be treated with caution, since non-linear effects may come into play. Further uncertainties arise due to uncertainties in the radiative forcing. The uncertainty in sulphate aerosol forcing is generally characterised in terms of the 1990 radiative forcing. Wigley and Smith (1998) and Smith et al. (2001) examined the effect of this uncertainty on future temperature change by varying the assumed 1990 sulphate radiative forcing by $0.6 \mathrm{Wm}^{-2}$ above and below a central value of $-1.1 \mathrm{Wm}^{-2}$. Reducing the sulphate forcing increased the 1990 to 2100 warming by 0 to $7 \%$ (depending on the scenario), while increasing the sulphate forcing decreased warming over the next century by a similar amount. The sensitivity to the uncertainty in sulphate forcing was found to be significantly less in the new scenarios than in the IS92a scenario; in the latter the sensitivity to sulphate forcing was twice as large as the largest value for the SRES marker scenarios. Therefore, the smaller future emissions of sulphur dioxide in the new scenarios significantly lowers the uncertainty in future global mean temperature change due to the uncertain value of present day sulphate aerosol forcing. The climate effects described here use the SRES scenarios as contained in Nakićenović et al. (2000). Any feedbacks on the socio-economic development path, and hence on emissions, as a result of these climate changes have not been included.

\subsubsection{Implications for temperature of stabilisation of greenhouse gases}

The objective of Article 2 of the United Nations Framework Convention on Climate Change (United Nations, 1992) is "to achieve stabilisation of greenhouse gas concentrations in the atmosphere at a level that would prevent dangerous anthropogenic interference with the climate system." This section gives an example of the possible effect on future temperature change of the stabilisation of greenhouse gases at different levels using carbon dioxide stabilisation as a specific example.
The carbon dioxide concentration stabilisation profiles developed by Wigley et al. (1996) (see also Wigley, 2000) commonly referred to as the WRE profiles, are used. These profiles indirectly incorporate economic considerations. They are also in good agreement with observed carbon dioxide concentrations up to 1999. Corresponding stabilisation profiles for the other greenhouses gases have not yet been produced. To illustrate the effect on temperature of earlier reductions in carbon dioxide emissions, results are also presented for the original stabilisation profiles referred to as the $\mathrm{S}$ profiles (Enting et al., 1994). The $\mathrm{S}$ profiles are, however, unrealistic because, for example, they require emissions and concentration values during the 1990s below those actually observed.

In order to define future radiative forcings fully, it is necessary to make assumptions about how the emissions or concentrations of the other gases may change in the future. In addition, it is necessary to have a base scenario against which the effect of the different stabilisation pathways may be assessed. The state of the science at present is such that it is only possible to give illustrative examples of possible outcomes (Wigley et al., 1996; Schimel et al., 1997; Mitchell et al., 2000).

To produce these examples, the SRES scenario A1B is used as the base scenario. $\mathrm{CO}_{2}$ concentrations for this scenario are close to the WRE $\mathrm{CO}_{2}$ profiles in terms of their implied past and near-future values, so our choice satisfies the underlying WRE assumption that emissions should initially follow a baseline trajectory. This is not the case for the $\mathrm{S}$ profiles, however, because as pointed out above, present day $\mathrm{CO}_{2}$ concentrations already exceed the values assumed for the $\mathrm{S}$ profiles. Note that the baseline scenario (A1B) is specified only out to 2100 . For stabilisation cases, emissions of non- $\mathrm{CO}_{2}$ gases are assumed to follow the A1B scenario out to 2100 and are thereafter held constant at their year 2100 level. For scenario A1B, this assumption of constant emissions from 2100 leads to stabilisation of the other gas concentrations at values close to their 2100 values. For gases with long lifetimes (such as $\mathrm{N}_{2} \mathrm{O}$ ) it takes centuries to reach stabilisation. In all cases, however, the net radiative forcing changes for the non- $\mathrm{CO}_{2}$ gases are small after 2100 and negligible after about 2200. Note that, in comparing the baseline case with the various stabilisation cases, the only gas that changes is $\mathrm{CO}_{2}$.

The models used to calculate the other gas concentrations and to convert concentrations and sulphur dioxide emissions to radiative forcing are the same as those used in Section 9.3.3. The simple climate model used is again that based on Wigley and Raper (1992) and Raper et al. (1996), tuned to the different AOGCMs using the CMIP2 data set (see Appendix 9.1).

The temperature consequences of the five WRE stabilisation profiles used, based on the assumptions described above and using the simple model ensemble (the average results from tuning the simple model to several AOGCMs), are shown in Figure 9.16. The temperature results for the $\mathrm{S}$ profiles are also included for comparison. The simple climate model can be expected to give results in good agreement to those that would be produced by the AOGCMs up to 2100. Thereafter the agreement becomes increasingly less certain and this increasing 


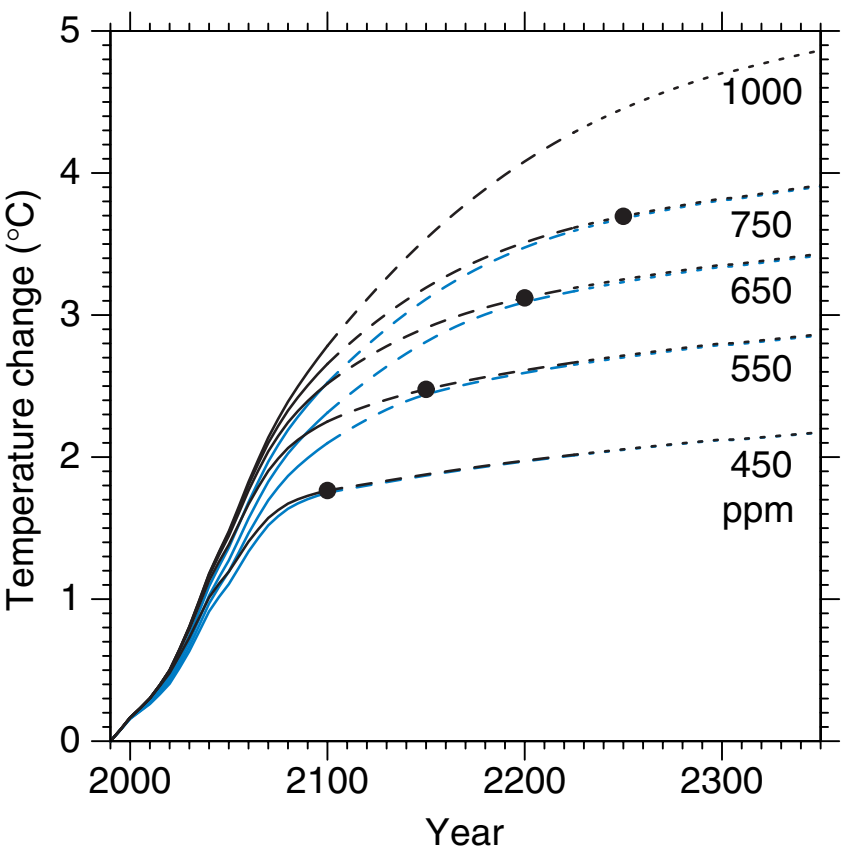

Figure 9.16: Simple model results: Projected global mean temperature changes when the concentration of $\mathrm{CO}_{2}$ is stabilised following the WRE profiles. For comparison, results with the original $\mathrm{S}$ profiles are also shown in blue (S1000 not available). The results are ensemble means produced by a simple climate model tuned to seven AOGCMs (see Appendix 9.1). The baseline scenario is scenario A1B, this is specified only to 2100 . After 2100 , the emissions of gases other than $\mathrm{CO}_{2}$ are assumed to remain constant at their A1B 2100 values. The projections are labelled according to the level of $\mathrm{CO}_{2}$ stabilisation (in ppm). The broken lines after 2100 indicate increased uncertainty in the simple climate model results beyond 2100 . The black dots indicate the time of $\mathrm{CO}_{2}$ stabilisation. The stabilisation year for the WRE1000 profile is 2375 .

uncertainty is indicated on the graph by the graduated broken lines. Indeed it has been shown in a comparison of results from the simple model and HadCM2 that the simple model underestimates the temperature change compared to HadCM2 on longer time-scales (Raper et al., 2001a). This is at least in part due to the fact that the HadCM2 effective climate sensitivity increases with time (see Section 9.3.4.1). The results in Figure 9.16 are consistent with the assumption of time-constant climate sensitivities, the average value being $2.8^{\circ} \mathrm{C}$.

Since sulphur dioxide emissions stabilise at 2100, the forcing from sulphate aerosols is constant thereafter. $\mathrm{CH}_{4}$ concentrations stabilise before 2200 , and the forcing change from $\mathrm{N}_{2} \mathrm{O}$ concentration changes after 2200 is less than 0.1 $\mathrm{Wm}^{-2}$. The continued increase in temperature after the time of $\mathrm{CO}_{2}$ stabilisation (Figure 9.16) is in part due to the later stabilisation of the other gases but is primarily due to the inertia in the climate system which requires several centuries to come into equilibrium with a particular forcing.

Temperature changes from 1990 to 2100 and from 1990 to 2350, for the simple climate model tuned to seven AOGCMs, are shown in Figure 9.17. These Figures give some indication
Table 9.3: Reduction in 1990 to 2100 temperature change, relative to the $A 1 B$ scenario, achieved by five WRE profiles across all seven simple model AOGCM model tunings. WRE1000 refers to stabilisation at a $\mathrm{CO}_{2}$ concentration of 1,000 ppm, etc.

\begin{tabular}{|l|ccccc|}
\hline Profile & WRE1000 & WRE750 & WRE650 & WRE550 & WRE450 \\
\hline $\begin{array}{l}\text { Percentage reduction } \\
\text { in temperature } \\
\text { change relative to A1B }\end{array}$ & $4-6 \%$ & $9-10 \%$ & $14-15 \%$ & $23-25 \%$ & $39-41 \%$ \\
\hline
\end{tabular}

of the range of uncertainty in the results due to differences in AOGCM response. Figure 9.17a also shows the temperature change for the baseline scenario, A1B. The percentage reductions in temperature change relative to the baseline scenario that the WRE profiles achieve by 2100 are given in Table 9.3. These range from 4 to $6 \%$ for the WRE1000 profile to 39 to $41 \%$ for the WRE450 profile. Note that these reductions are for stabilisation of $\mathrm{CO}_{2}$ concentrations alone.

Although only $\mathrm{CO}_{2}$ stabilisation is explicitly considered here, it is important to note that the other gases also eventually stabilise in these illustrations. The potential for further reductions in warming, both up to 2100 and beyond, through non- $\mathrm{CO}_{2}$ gases, depends on whether, in more comprehensive scenarios (when such become available), their stabilisation levels are less than the levels assumed here.

Only one AOGCM study has considered the regional effects of stabilising $\mathrm{CO}_{2}$ concentrations (Mitchell et al., 2000). HadCM2, which has an effective climate sensitivity in the middle of the IPCC range (Table 9.1), was run with the S550 ppm and S750 ppm stabilisation profiles ("S profiles"; Enting $e t$ al., 1994; Schimel et al., 1997). Simulations with a simple climate model (Schimel et al., 1997) indicate that the global mean temperature response in these profiles is likely to differ by no more than about $0.2^{\circ} \mathrm{C}$ from the equivalent WRE profiles (Wigley et al., 1996; see Figure 9.16), though the maximum rate of temperature change is likely to be lower with the $\mathrm{S}$ profiles. Global mean changes in the AOGCM experiments are similar to those in Schimel et al. (1994). Note that the AOGCM experiments consider stabilisation of $\mathrm{CO}_{2}$ concentrations only, and do not take into account changes in other gases, effectively assuming that concentrations of other gases are stabilised immediately. To allow for ongoing increases in other greenhouse gases, one would have in practice to reduce $\mathrm{CO}_{2}$ to even lower levels to obtain the same level of climate change. For example, in the IS92a scenario, other trace gases contribute $1.3 \mathrm{Wm}^{-2}$ to the radiative forcing by 2100 . If the emissions of these gases were to continue to increase as in the IS92a scenario, then $\mathrm{CO}_{2}$ levels would have to be reduced by about $95 \mathrm{ppm}$ to maintain the same level of climate change in these experiments.

Changes in temperature and precipitation averaged over five sub-continental regions at 2100 were compared to those in a baseline scenario based on $1 \% / y r$ increase in $\mathrm{CO}_{2}$ concentrations from 1990. With both stabilisation profiles, there were significant reductions in the regional temperature changes but the significance of the regional precipitation changes depended on location and season. The response of AOGCMs to idealised stabilisation profiles is discussed in Section 9.3.4.4. 
(a) Temperature change from 1990 to 2100

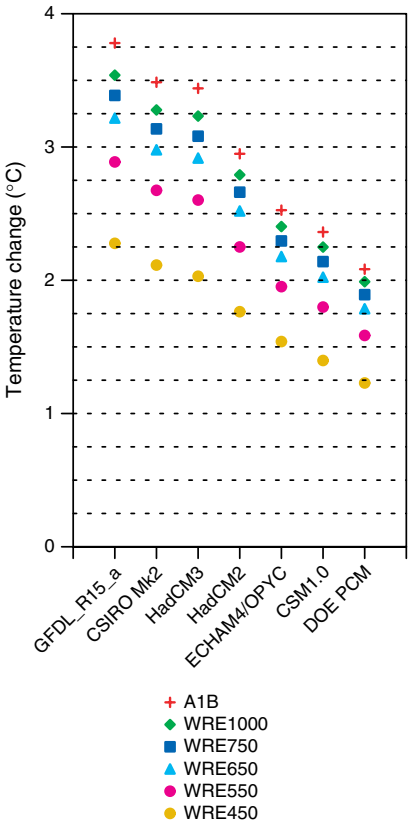

(b) Temperature change from 1990 to 2350

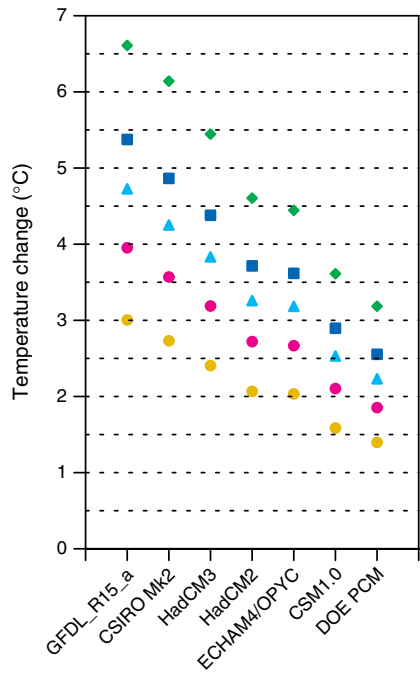

- WRE1000 - WRE750 $\triangle$ WRE650 - WRE550 (c) Temperature change from 1990 to 2100

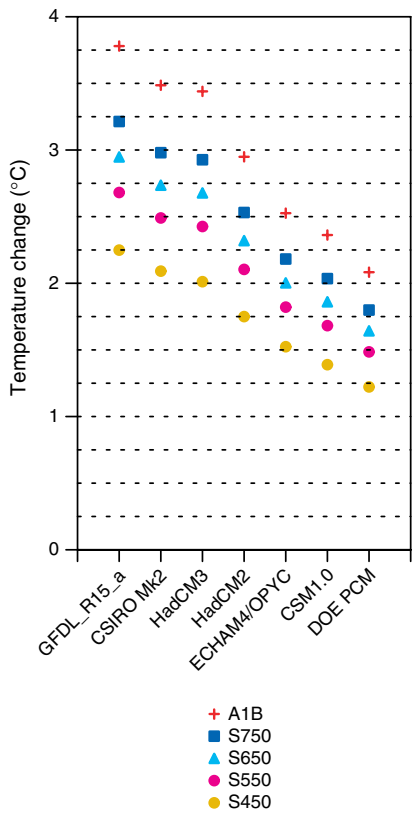

(d) Temperature change from 1990 to 2350

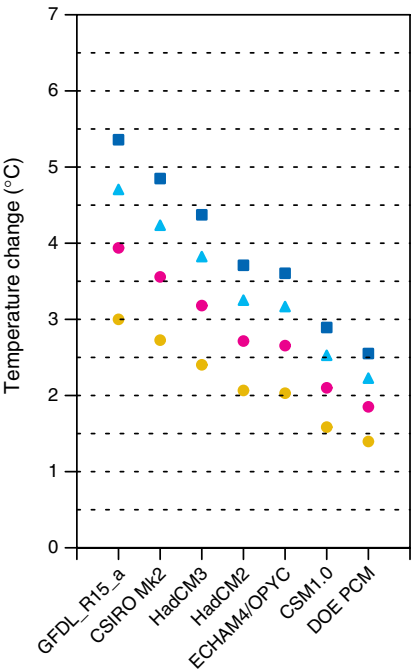

Figure 9.17: Simple model results: Temperature change (a) from 1990 to 2100 and (b) from 1990 to 2350, resulting from the five WRE stabilisation profiles, using the simple climate model tuned to different AOGCMs as indicated on the bottom axis. (c) and (d) show the corresponding results for the four $\mathrm{S}$ profiles. The underlying assumptions are the same as those for Figure 9.15. For comparison, temperature changes for the base scenario A1B are also shown in (a) and (c).

\subsubsection{Factors that Contribute to the Response}

\subsubsection{Climate sensititivity}

A variety of feedback processes operate in the climate system (Chapter 7) to determine the response to changes in radiative forcing. The climate sensitivity (see Section 9.2.1) is a broad measure of this response. Ideally, a coupled AOGCM's climate sensitivity would be obtained by integrating the model to a new climate equilibrium after doubling the $\mathrm{CO}_{2}$ concentration in the model atmosphere. Since this requires a lengthy integration, climate sensitivities are usually estimated with atmospheric GCMs coupled to mixed-layer upper ocean models, for which the new equilibrium is obtained in decades rather than millennia. Equilibrium climate sensitivities for models in current use are compared with the results reported in the SAR. A related measure, the effective climate sensitivity, is obtained from nonequilibrium transient climate change experiments.

\section{Equilibrium climate sensitivity from AGCMs coupled to mixed- layer upper ocean models}

The blue diamonds in Figure 9.18 give the equilibrium climate sensitivity and the associated percentage change in global mean precipitation rate (sometimes termed the hydrological sensitivity) for seventeen equilibrium mixed-layer model calculations documented in Table 6.3 of the SAR (Kattenberg et al., 1996). Table 9.4 gives the average sensitivity of the seventeen models as $3.8^{\circ} \mathrm{C}$ for temperature and $8.4 \%$ for precipitation, with a standard deviation or "inter-model scatter" of $0.78^{\circ} \mathrm{C}$ and $2.9 \%$, respectively. LeTreut and McAvaney (2000) provide a recent compilation of climate sensitivities for mixed-layer models and this information has been updated in Table 9.1 under the column headed "equilibrium climate sensitivity". These results, from fifteen models in active use, are represented by the red triangles in Figure 9.18. The associated statistics are given in Table 9.4 where the mean and standard deviation for temperature are 3.5 and $0.92^{\circ} \mathrm{C}$, and for precipitation are 6.6 and $3.7 \%$.

According to Table 9.4 and Figure 9.18, the average climate sensitivity, as estimated from AGCMs coupled to mixed-layer ocean models, has decreased slightly from about 3.8 to $3.5^{\circ} \mathrm{C}$ since the SAR. The inter-model standard deviation has increased and the range has remained essentially the same. The associated hydrological sensitivity has decreased from 8.4 to $6.6 \%$ but the inter-model standard deviation has increased. As explained in Section 9.2, these climate sensitivity values are not altered by the lower value for the radiative forcing change for doubled $\mathrm{CO}_{2}$ discussed in Chapter 6.

These results indicate slightly lower average values of sensitivity in models in current use compared with the SAR. Although more recent models attempt to incorporate improvements in our ability to simulate the climate system, these mean results do not in themselves provide a clear indication that modelled climate sensitivity has decreased. In particular, the inter-model scatter has increased slightly, the range of results is not much changed, the differences are not statistically significant, and the reasons for the modest decrease in average sensitivity have not been identified.

\section{Climate sensitivity from AGCMs coupled to full OGCMs}

Because of the long time-scales associated with deep ocean equilibration, the direct calculation of coupled model equilibrium 


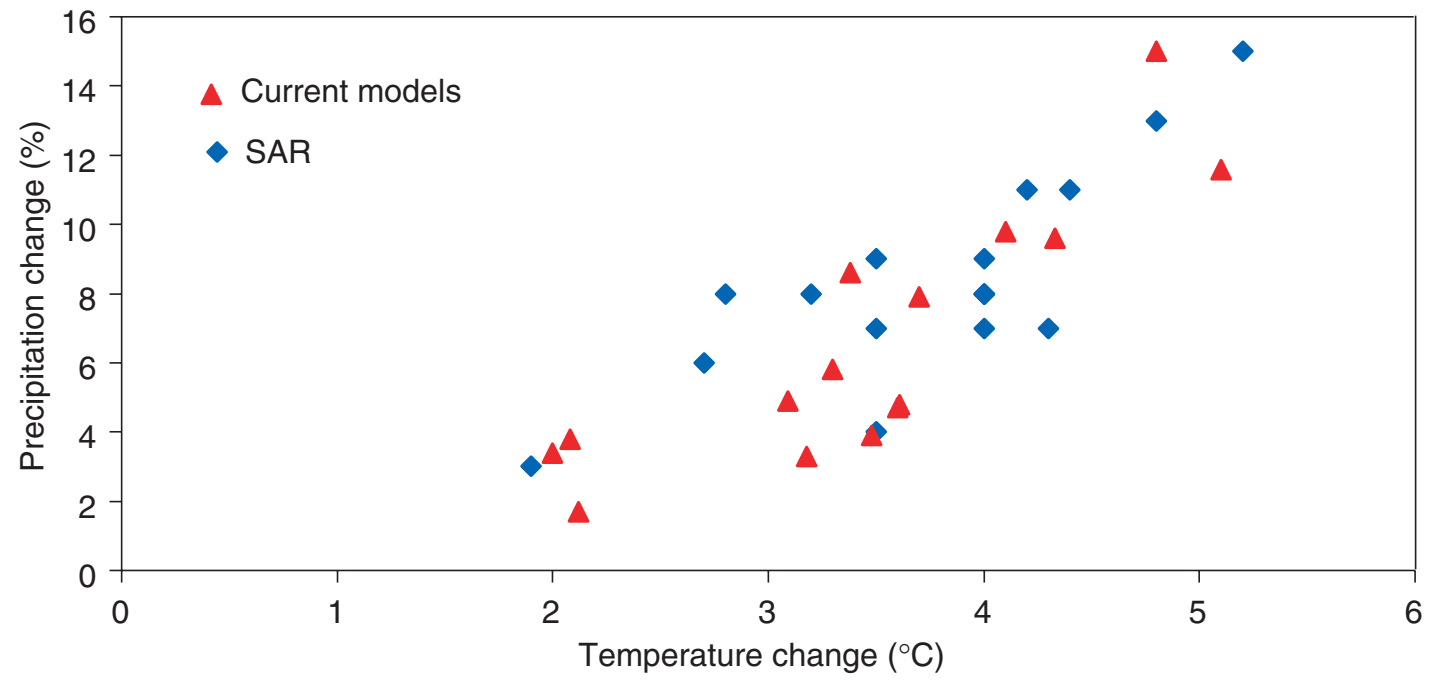

Figure 9.18: Equilibrium climate and hydrological senstitivies from AGCMs coupled to mixed-layer ocean components; blue diamonds from the SAR, red triangles from models in current use (LeTreut and McAvaney, 2000 and Table 9.1).

Table 9.4: Statistics of climate and hydrological sensitivity for mixed-layer models

\begin{tabular}{|l|c|c|c|c|c|c|c|}
\hline Source & No. of models & \multicolumn{3}{|c|}{ Temperature $\left({ }^{\circ} \mathbf{C}\right)$} & \multicolumn{3}{c|}{ Precipitation (\%) } \\
\hline & & Mean & $\begin{array}{c}\text { Standard } \\
\text { deviation }\end{array}$ & Range & Mean & $\begin{array}{c}\text { Standard } \\
\text { deviation }\end{array}$ & Range \\
\hline SAR & 17 & 3.8 & 0.78 & $1.9 / 5.2$ & 8.4 & 2.9 & $3 / 15$ \\
\hline Current models & 15 & 3.5 & 0.92 & $2.0 / 5.1$ & 6.6 & 3.7 & $2 / 15$ \\
\hline
\end{tabular}

temperature change for doubled $\mathrm{CO}_{2}$ requires an extended simulation and a considerable commitment of computer resources. One such calculation has been performed (Stouffer and Manabe, 1999, Table 9.1); a 4,000 year simulation with stabilisation at $2 \times \mathrm{CO}_{2}$, and a 5,000 year simulation with stabilisation at $4 \times \mathrm{CO}_{2}$. Figure 9.19 displays the temperature for the first 500 years of these simulations (the red curves) together with stabilisation results from other models discussed further below. The calculation shows that: (1) some 15 to 20 centuries are required for the coupled model to attain a new equilibrium after the forcing is stabilised, (2) for the $2 \times \mathrm{CO}_{2}$ case, the temperature change ultimately increases to $4.5^{\circ} \mathrm{C}$ for the GFDL_R15_a model, which exceeds the $3.9^{\circ} \mathrm{C}$ value obtained when a mixed-layer ocean is used to estimate the climate sensitivity and, (3) the $4 \times \mathrm{CO}_{2}$ equilibrium temperature change is very nearly twice that of the $2 \times \mathrm{CO}_{2}$ equilibrium temperature change for this model. In this case the mixed-layer value of climate sensitivity is resonably close to the full climate model value. The difference between coupled model and mixed-layer sensitivities for other models is unknown.

\section{Effective climate sensitivity}

The term effective climate sensitivity (Murphy, 1995) as defined in Section 9.2.1 is a measure of the strength of the feedbacks at a particular time in a transient experiment. It is a function of climate state and may vary with time. Watterson (2000) calculates the effective climate sensitivity from several experiments with different versions of an AOGCM. The results show considerable variability, particularly near the beginning of the integrations when the temperature change is small. That study nevertheless concludes that the effective climate sensitivity is approximately constant and close to the appropriate equilibrium result. However, estimates of effective climate sensitivity obtained from the HadCM2 model range from about $2.7^{\circ} \mathrm{C}$ at the time of stabilisation at $2 \times \mathrm{CO}_{2}$ to about $3.8^{\circ} \mathrm{C}$ after 900 years (Raper et al., 2001a). Senior and Mitchell (2000) implicate time-dependent cloudfeedbacks associated with the slower warming of the Southern Ocean in that model as the cause for this variation in time. The effective climate sensitivity of this climate model is initially considerably smaller than the equilibrium sensitivity obtained with a mixed-layer ocean. As the coupled model integration approaches a new equilibrium, the effective climate sensitivity increases and appears to be approaching the equilibrium climate sensitivity.

If effective climate sensitivity varies with climate state, estimates of climate sensitivity made from a transient simulation may not reflect the ultimate warming the system will undergo. The use of a constant climate sensitivity in simple models will lead to inconsistencies which depend on the value of sensitivity chosen. This feature deserves further study. 
(a)
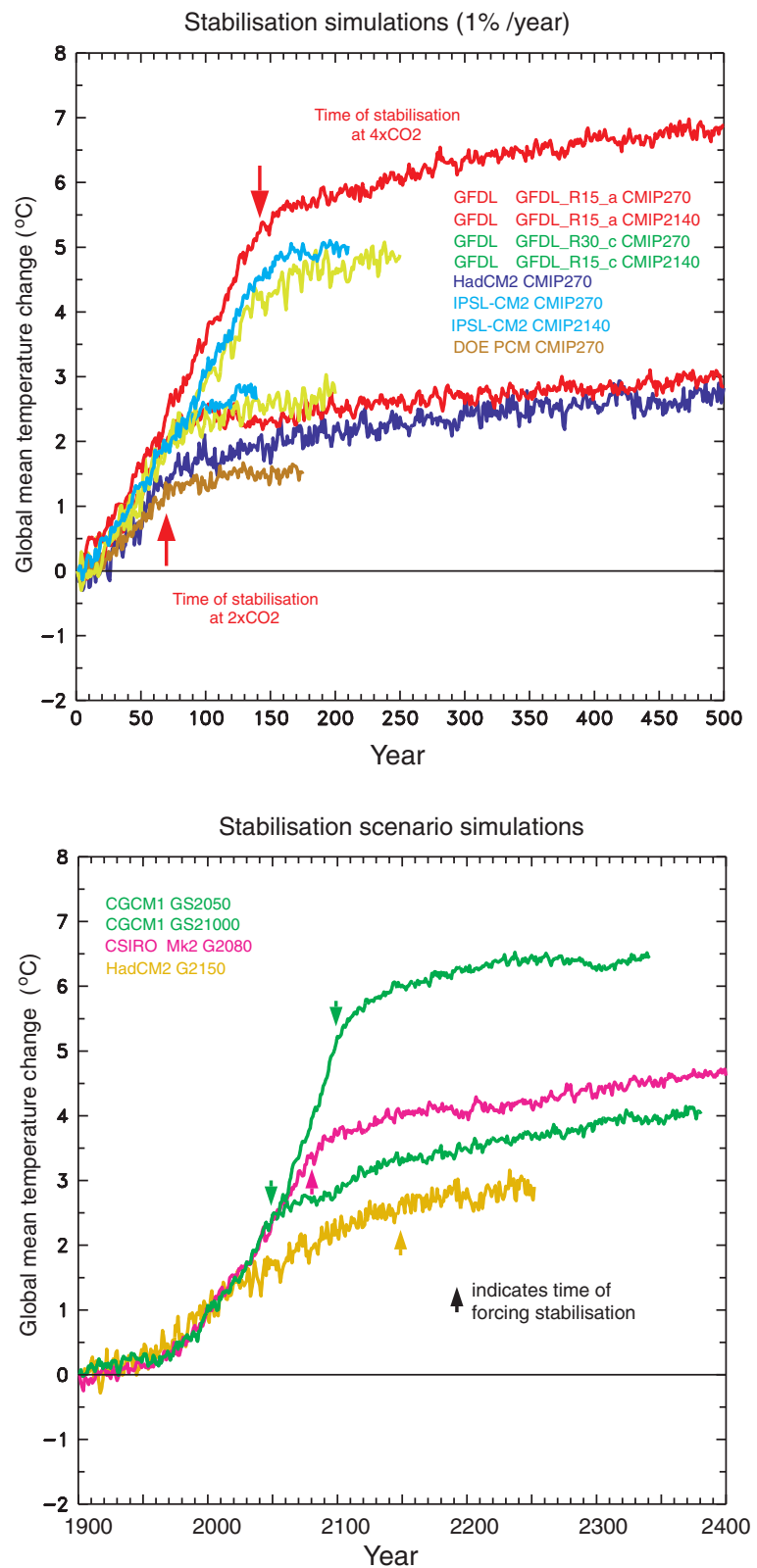

(b)
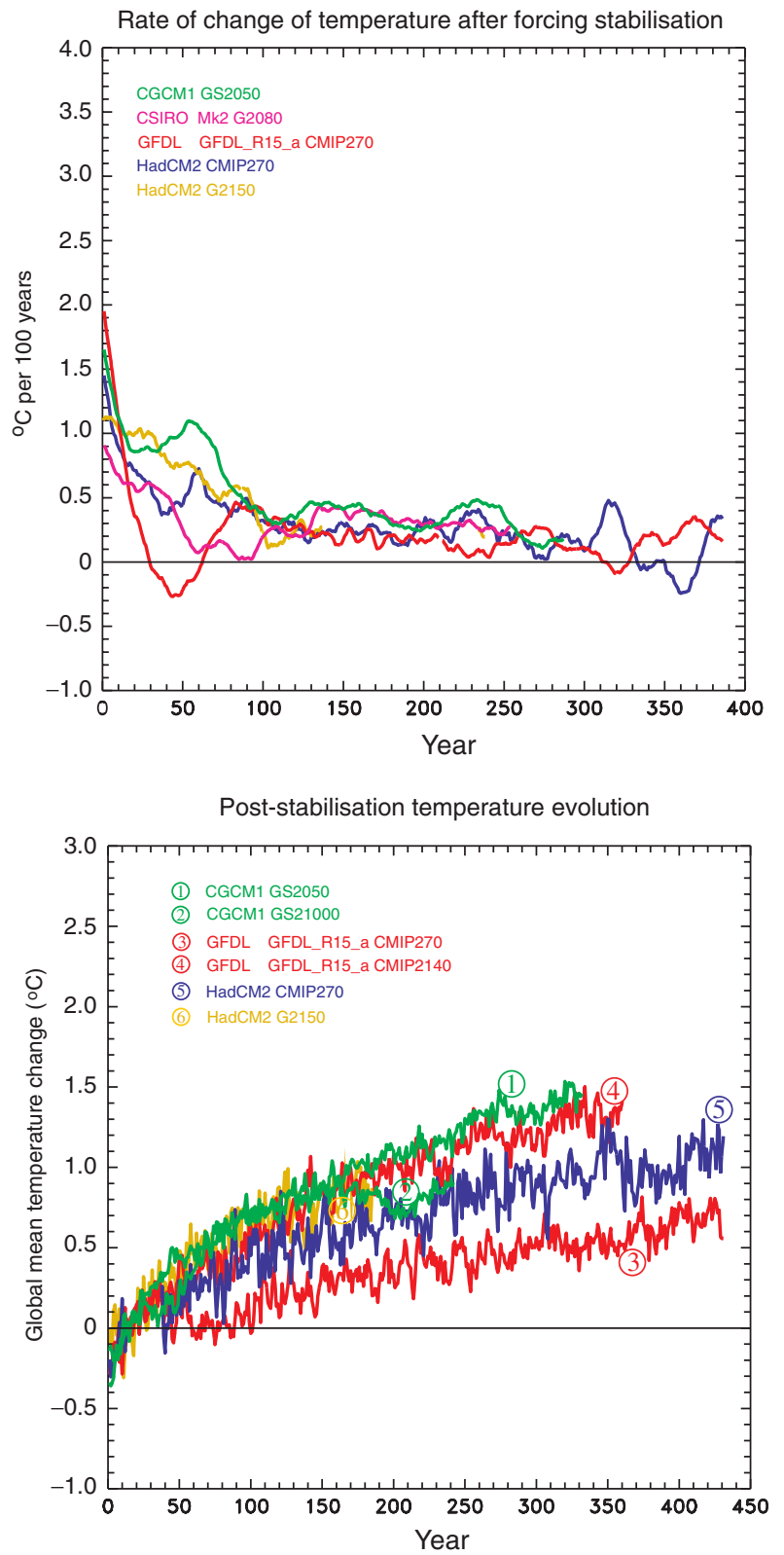

Figure 9.19: (a) AOGCM stabilisation simulations. Upper panel: $1 \% / \mathrm{yr}$ simulations to stabilisation at and near $2 \times$ and $3 \times \mathrm{CO}_{2}$. Lower panel: stabilisation experiments starting with historical greenhouse gas forcing up to present day, then going up to $2 \times$ or $3 \times \mathrm{CO}_{2}$ concentrations following the IS92a scenario, then stabilising in the years 2050 and 2100. (b) Post-stabilisation temperature evolution. Upper panel: Rate of change of the temperature. Lower panel: Temperature evolution after the stabilisation of the greenhouse gas concentration.

\section{Summary}

The climate sensitivity is a basic measure of the response of the climate system to a change in forcing. It may be measured in several ways as discussed above. The equilibrium climate sensitivity, that is, the range of the surface air temperature response to a doubling of the atmospheric $\mathrm{CO}_{2}$ concentration, was estimated to be between 1.5 and $4.5^{\circ} \mathrm{C}$ in the SAR (Kattenberg et al., 1996). That range still encompasses the estimates from the current models in active use.
9.3.4.2 The role of climate sensitivity and ocean heat uptake

Earlier (Section 9.3.1), it was noted that the climate response varies from model to model even when the radiative forcing used to drive the models is similar. This difference in the climate models' response is mainly the result of differing climate sensitivities and differing rates of heat uptake by the oceans in each model, although differences in the AOGCM radiative forcing for a given $\mathrm{CO}_{2}$ concentration also have a small effect (see Chapter 6, Section 6.3). 


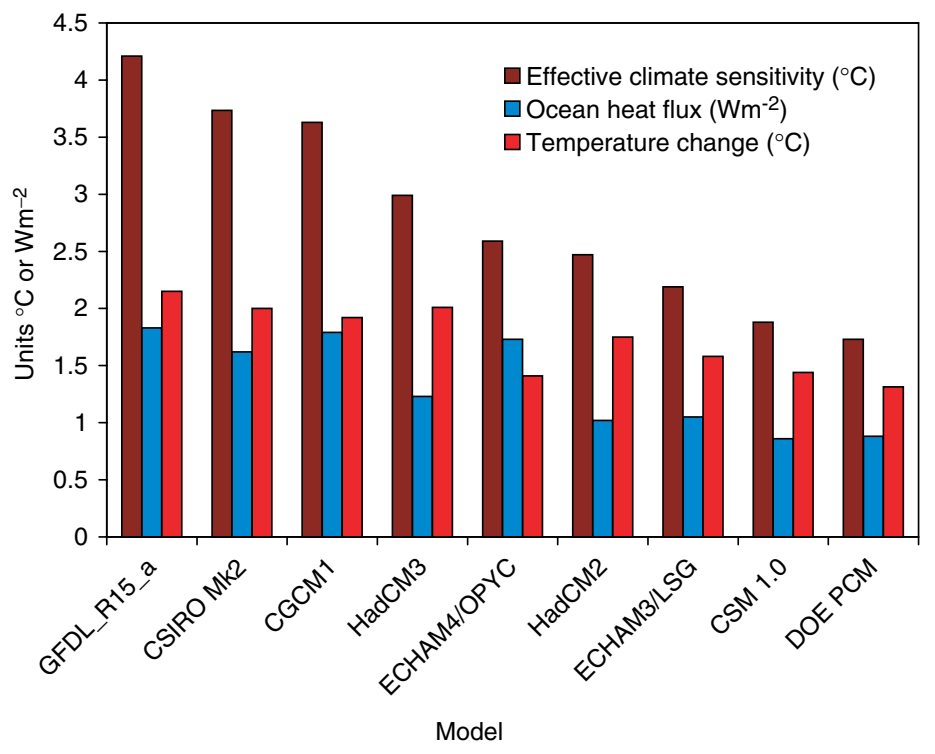

Figure 9.20: Comparison of CMIP2 model results for 20-year average values centred on year 70 , the time of $\mathrm{CO}_{2}$ doubling. Values are shown for the effective climate sensitivity, the net heat flux across the ocean surface multiplied by the ocean fraction and the global mean temperature change (TCR).

The effective climate sensitivity and ocean heat uptake are compared by Raper et al. (2001b) using the CMIP2 data set $\left(1 \% / \mathrm{yr} \mathrm{CO}_{2}\right.$ increase to doubling). The effective climate sensitivities around the time of $\mathrm{CO}_{2}$ doubling (average for the years 61 to 80 ), when the signal is strongest, agree reasonably well with the mixed-layer equilibrium climate sensitivities given in Figure 9.20. Results are shown for various models in Figure 9.20 It is evident that the models with high effective climate sensitivity also tend to have a large net heat flux into the ocean. This oceanic heat flux causes a delay in the climate response. The relationship between the effective climate sensitivity and the oceanic heat uptake was first described by Hansen et al. $(1984,1985)$ using a box diffusion model. Raper et al. (2001b) show that an additional oceanfeedback is possibly associated with the warming and freshening of the high latitude surface waters that enhances this relationship. Details of the individual model's sub-grid scale parametrizations also affect both the effective climate sensitivity and the oceanic heat uptake (Weaver and Wiebe, 1999). The evident relationship between effective climate sensitivity and ocean heat uptake leads to the transient climate response (TCR) having a smaller spread among the model results than the climate models' climate sensitivity alone would suggest (see Section 9.3.1). Since the oceanic heat uptake is directly related to the thermal expansion, the range for thermal expansion is correspondingly increased due to the compensation noted above (see Chapter 11 for a complete discussion of sea level rise).

\subsubsection{Thermohaline circulation changes}

In the SAR, it was noted that the thermohaline circulation (THC) weakens as $\mathrm{CO}_{2}$ increases in the atmosphere in most coupled climate model integrations. The weakening of the THC is found in both the Northern and Southern Hemispheres. The amount of weakening varied from model to model, but in some cases it was noted that the THC in the North Atlantic stopped completely (Manabe and Stouffer, 1994; Hirst 1999). The weakening of the THC in the Atlantic Ocean results in a reduction of the poleward heat transport that in turn leads to a minimum in the surface warming in the northern North Atlantic Ocean and/or in the circumpolar Ocean (see Section 9.3.2). The reduction in the warming in the North Atlantic region touches the extreme northeastern part of North America and north-west Europe. The shutting off of the THC in either hemisphere could have long-term implications for climate. However, even in models where the THC weakens, there is still a warming over Europe. For example, in all AOGCM integrations where the radiative forcing is increasing, the sign of the temperature change over north-west Europe is positive (see Figure 9.10).

Figure 9.21 shows a comparison of the strength of the THC through a number of transient experiments with various models and warming scenarios over the 21st century. The initial (control state) absolute strength of the Atlantic thermohaline circulation (THC) varies by more than a factor of 2 between the models, ranging from 10 to $30 \mathrm{~Sv}\left(1 \mathrm{~Sv}=10^{6} \mathrm{~m}^{3} \mathrm{~s}^{-1}\right)$. The cause of this wide variation is unclear, but it must involve the sub-grid parametrization schemes used for mixing in the oceans (Bryan, 1987) and differences in the changes of the surface fluxes. The sensitivity of the THC to changes in the radiative forcing is also quite different between the models. Generally as the radiative forcing increases, most models show a reduction of THC. However, some models show only a small weakening of the THC and one model (ECHAM4/OPYC; Latif et al., 2000) has no weakening in response to increasing greenhouse gases, as does the NCAR CSM as documented by Gent (2001). The exact reasons for the difference in the THC responses are unknown, but the role of the surface fluxes is certainly part of the reasons for the differences in the response (see below).

Stocker and Schmittner (1997), using an intermediate complexity model, found that the North Atlantic THC shut-down when the rate of $1 \% / y$ of $\mathrm{CO}_{2}$ increase was held fixed for approximately 100 years. This is in agreement with the earlier AOGCM study of Manabe and Stouffer (1994), where the THC shut-down in an integration where the $\mathrm{CO}_{2}$ concentration increased by $1 \% / \mathrm{yr}$ to four times its initial value. In integrations where the $\mathrm{CO}_{2}$ stabilised at doubling, the THC did not shut-down in either study (Stocker and Schmittner 1997; Manabe and Stouffer 1994). Furthermore, in the Manabe and Stouffer (1994) AOGCM where the $\mathrm{CO}_{2}$ is stabilised at four times its normal value, the THC recovers to the control integration value around model year 2300 . A recent study (Stouffer and Manabe, 1999) found that the amount of weakening of the THC by the time of $\mathrm{CO}_{2}$ doubling is a function of the rate of $\mathrm{CO}_{2}$ increase and not the absolute increase in the radiative forcing. They found the slower the rate of increase, the more the weakening of the $\mathrm{THC}$ by the time of $\mathrm{CO}_{2}$ doubling.

The evolution of the THC in response to future forcing scenarios is a topic requiring further study. It should be noted in particular that these climate model experiments do not currently include the possible effects of significant freshwater input arising from changes in land ice sheets (Greenland and Antarctic ice caps) and mountain glaciers, which might well lead to bigger reductions 




Figure 9.21: Simulated water-volume transport change of the Atlantic "conveyor belt" (Atlantic overturning) in a range of global warming scenarios computed by different climate research centres. Shown is the annual mean relative to the mean of the years (1961 to 1990) (Unit: SV, $\left.10^{6} \mathrm{~m}^{3} \mathrm{~s}^{-1}\right)$. The past forcings are only due to greenhouse gases and aerosols. The future-forcing scenario is the IS92a scenario. See Table 9.1 for more information on the individual models used here.

in the THC. It is too early to say with confidence whether irreversible shut-down of the THC is likely or not, or at what threshold it might occur. Though no AOGCM to date has shown a shut-down of the THC by the year 2100 , climate changes over that period may increase the likelihood during subsequent centuries, though this is scenario-dependent. The realism of the representation of oceanic mechanisms involved in the THC changes also needs to be carefully evaluated in the models.

\section{Role of the surface fluxes}

The role of heat, fresh water and momentum fluxes in weakening the North Atlantic THC as a consequence of increasing atmospheric $\mathrm{CO}_{2}$ concentration has been studied in two different AOGCMs (ECHAM3/LSG, Mikolajewicz and Voss, 2000; and GFDL_R15_b, Dixon et al., 1999). In both these studies (Figure 9.22), two baseline integrations are performed; a control integration in which the $\mathrm{CO}_{2}$ is held fixed, and a perturbation integration in which the $\mathrm{CO}_{2}$ is increasing. The water fluxes from both of these integrations are archived and used as input in two new integrations.

In the first integration, the atmospheric $\mathrm{CO}_{2}$ concentration is held fixed and the fresh water fluxes into the ocean are prescribed as those obtained from the perturbation integration. In the second integration, the $\mathrm{CO}_{2}$ increases as in the perturbation integration and the water fluxes are prescribed to be the fluxes from the control integration (see Table 9.5). In this way, the relative roles of the fresh water and heat fluxes can be evaluated (Figure 9.22).
Table 9.5: The THC-sensitivity experiments.

\begin{tabular}{|c|l|l|l|}
\hline Experiment & $\mathbf{C O}_{2}$ concentration & Freshwater flux & Wind stress \\
\hline FSS & fixed present day & simulated & simulated \\
\hline ISS & increasing & simulated & simulated \\
\hline IFS & increasing & from FSS & simulated \\
\hline FIS & fixed present day & from ISS & simulated \\
\hline FSI & fixed present day & simulated & from ISS \\
\hline IFF & increasing & from FSS & from FSS \\
\hline
\end{tabular}

\subsubsection{Time-scales of response}

As mentioned earlier, the basis of the experiments discussed in the SAR is a transient increase of greenhouse gases throughout the integration. In the model integrations presented in this section, the $\mathrm{CO}_{2}$ concentration increases up to a certain value (e.g., a doubling of the $\mathrm{CO}_{2}$ concentration) and then remains constant for the remainder of the integration. Since this type of integration involves integrating the model for very long time periods (at least several centuries) only a few integrations have been performed using AOGCMs. Furthermore, no standard emission scenarios have been used for forcing these model runs and most have used idealised stabilisation values $\left(2 \times \mathrm{CO}_{2}\right.$ or $3 \times \mathrm{CO}_{2}$ or $4 \times \mathrm{CO}_{2}$ for example). Again, in these integrations, the $\mathrm{CO}_{2}$ changes represent the radiative forcing changes of all the greenhouse gases. Results from the models of intermediate complexity are used to help understand the coupled model results, or in some cases, to explore areas where 

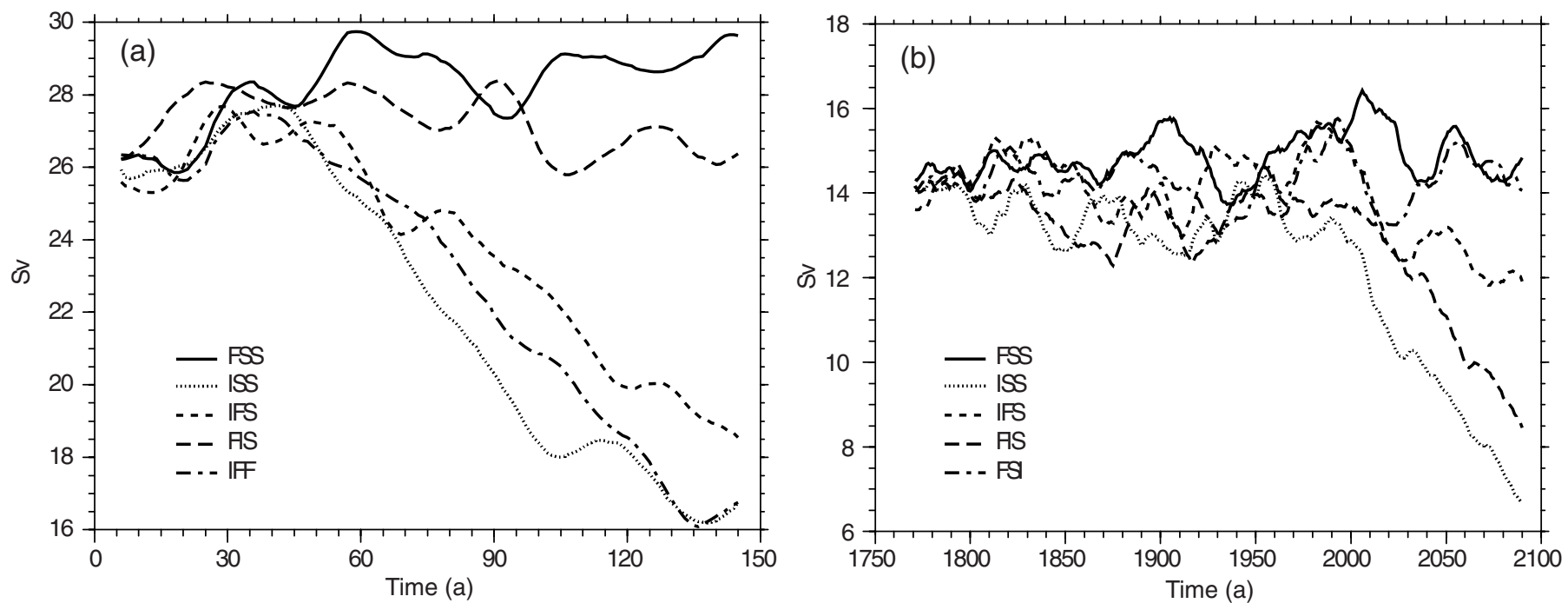

Figure 9.22: Time-series of the zonally integrated Atlantic mass transport stream function at $30^{\circ} \mathrm{N}$ and $1500 \mathrm{~m}$ depth, close to the maximum of the stream function simulated by the (a) ECHAM3/LSG model and the (b) GFDL_R15_b model. For a description of the experiments see Table 9.5.
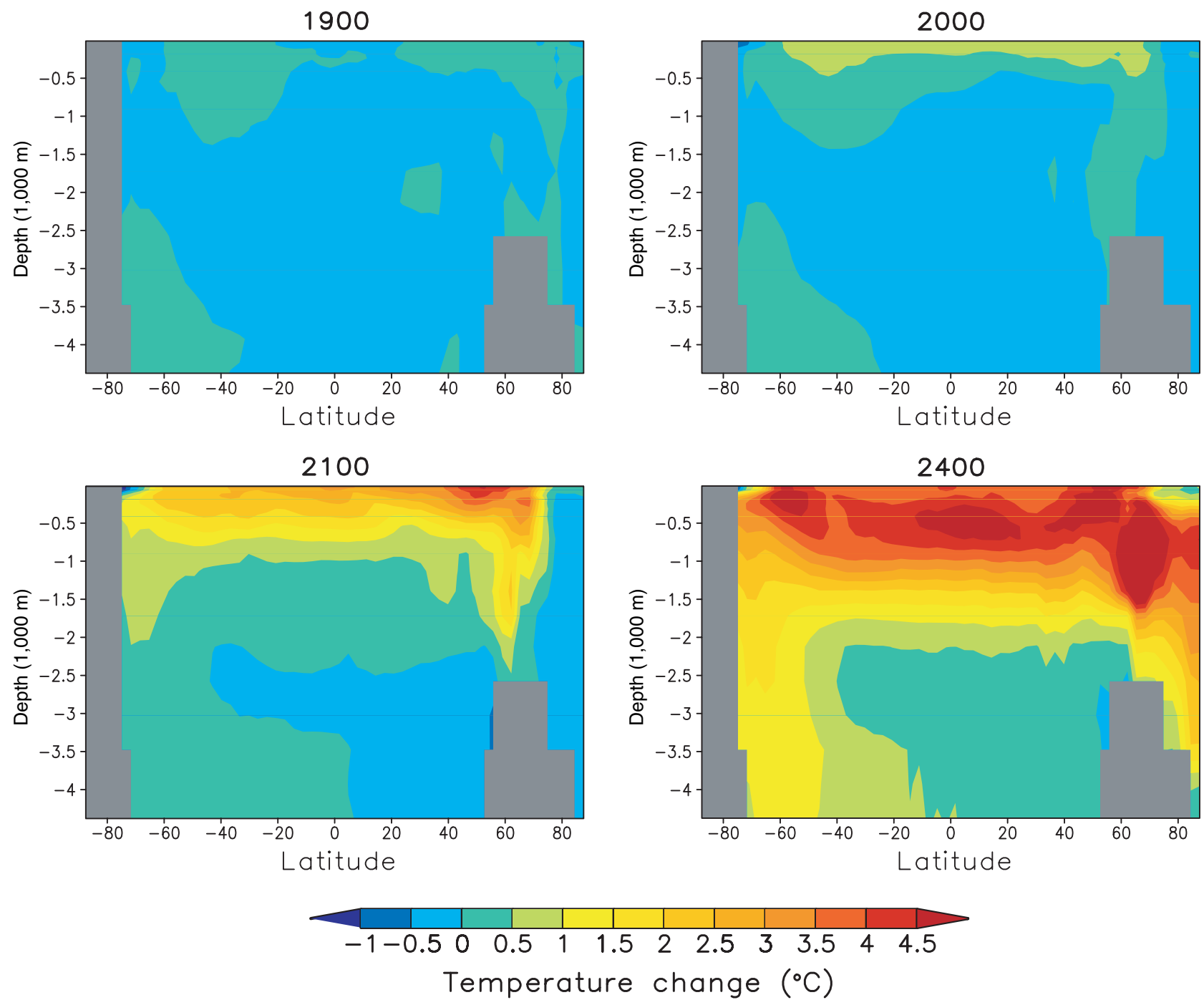

Figure 9.23: Cross-sections of ocean temperature change in the CSIRO Mk2 model stabilisation $\left(3 \times \mathrm{CO}_{2}\right)$ experiment $(\mathrm{Hirst}$, 1999). 

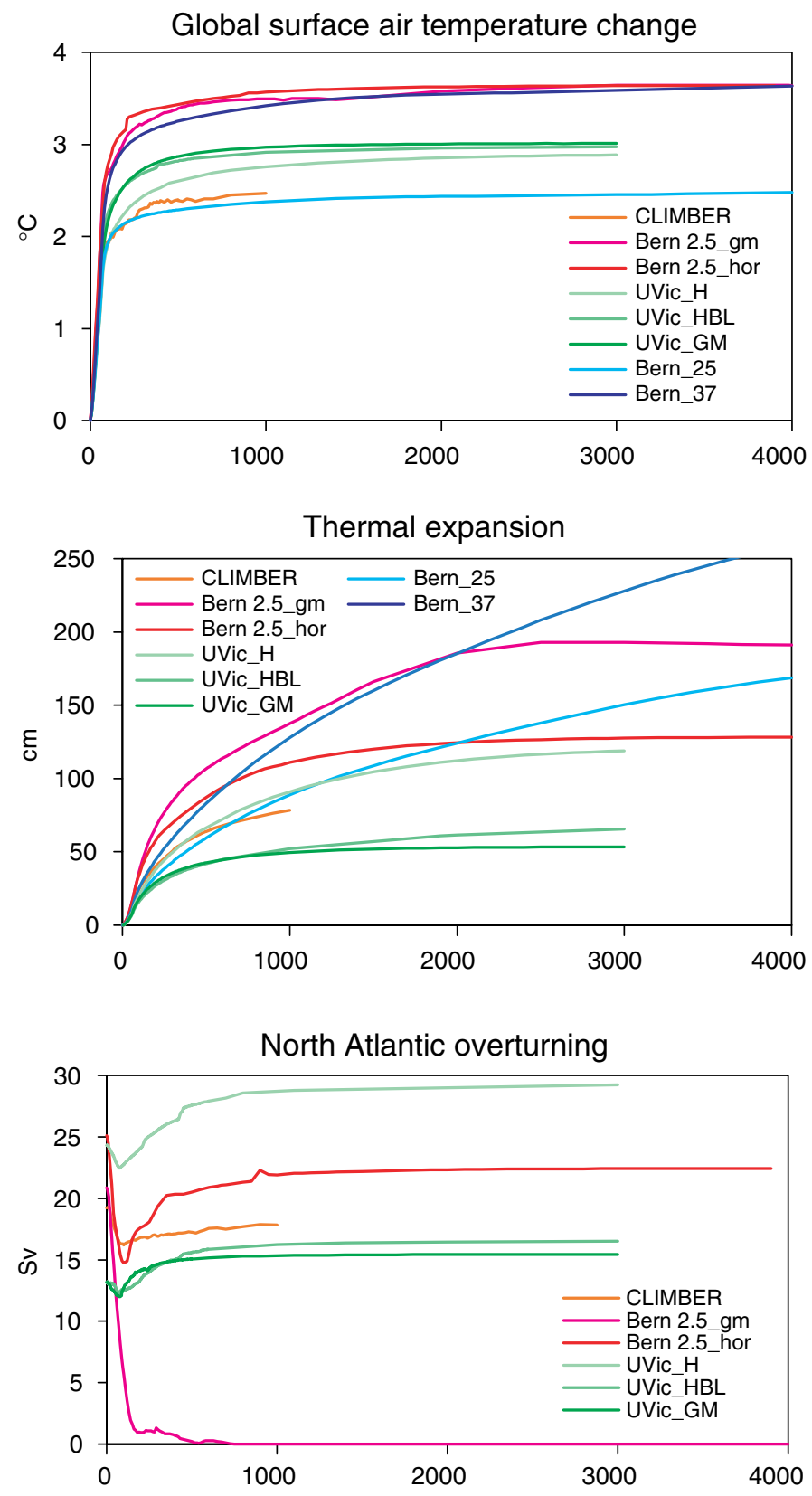

Figure 9.24: Global mean temperature change, thermal expansion and North Atlantic overturning for a number of models of intermediate complexity. The models have been forced by $1 \%$ increase of $\mathrm{CO}_{2}$ until doubling, then the $\mathrm{CO}_{2}$ concentration has been kept constant.

AOGCM integrations do not exist. Experiments where the coupled system is allowed time to reach equilibrium with the radiative forcing clearly show the response times of its various components.

Even after the radiative forcing becomes constant, the surface air temperature continues to increase for many centuries (Figure 9.19) as noted in Section 9.3.4.1. The rate of warming after stabilisation is relatively small $\left(<0.3^{\circ} \mathrm{C}\right.$ per century, Figure $\left.9.19 \mathrm{~b}\right)$; however, the total warming after the radiative forcing stabilises can be significant (more than $1^{\circ} \mathrm{C}$ ) because the warming continues for a long time period (Figure 9.19b). From Figure 9.19, one notes that the rate of warming after stabilisation varies from model to model.
The slow rate of surface air temperature increase occurs as the heat anomaly slowly penetrates to depth in the ocean (Figure 9.23). The rate of penetration is dependent on the model's vertical mixing both resolved by the model's grid and by the sub-grid scale parametrizations. The effect of the oceanic mixing parametrizations on the coupled model response has been investigated using climate models of intermediate complexity (Figure 9.24): CLIMBER - Ganopolski et al. (2001); Bern 2.5_gm, Bern 2.5_hor - Stocker et al. (1992); Uvic - Fanning and Weaver (1996), Weaver et al. (1998), Wiebe and Weaver (2000); Bern_25, Bern_37 Siegenthaler and Joos (1992), Joos et al. (1996). The effect on the response of the global mean surface air temperature, thermal expansion (see Chapter 11, Section 11.5.4.1 for a more complete discussion) and THC can be seen by comparing the results obtained from Stocker's (Bern 2.5) models and the Uvic models (Weaver and Wiebe, 1999). The sub-grid scale mixing parametrizations vary in the AOGCMs, accounting for much of the difference in the rate of surface warming (as seen in Figure 9.19b).

The thermohaline circulation (THC) response is more complex than that of the surface air temperature in the stablisation integrations (compare Figure 9.19a with Figure 9.25, for example). Typically the THC weakens as the radiative forcing increases (Section 9.3.4.3). After the radiative forcing stabilises, the THC recovers to its control integration value. The initial weakening is caused by the warming of the mixed layer in the ocean and the increase in the freshwater flux in high latitudes. As the radiative forcing stablises, the tendency for the surface fluxes to weaken the THC is balanced by the changes in the ocean heat and water transports and vertical structure. It is found that the time-scale for this recovery varies from model to model (about a century to multicenturies). Again it is likely that differences in the oceanic mixing are the cause for the differences in the recovery time.

The time rate of change in the radiative forcing also affects both the weakening and recovery of the THC (Figure 9.25). In the GFDL_R15_a model when the $\mathrm{CO}_{2}$ increased at a rate of $1 \% / \mathrm{yr}$ to doubling, the THC continued to weaken for 70 years after the point at which the $\mathrm{CO}_{2}$ was held constant at the doubled value (year 70). In a second integration, the $\mathrm{CO}_{2}$ increased at a rate of $0.25 \% / \mathrm{yr}$ to doubling. In this integration, the THC does not weaken after the doubling point (Manabe and Stouffer, 1994), indicating that the behaviour of the THC response is highly dependent on the rate that the radiative forcing changes (Figure 9.25).

Finally, it is important to note that the transient THC response (i.e., the weakening) is quite different from the equilibrium response of the THC (i.e., little change). This fact makes the interpretation of comparisons between palaeo-proxy data and coupled model results presented here difficult, since one needs to know the details of the changes in the radiative forcing and resolve relatively small time-scales in the proxy record.

\subsubsection{Changes in Variability}

The capability of models to simulate the large-scale variability of climate, such as the El Niño-Southern Oscillation (ENSO) (a major source of global interannual variability) has improved substantially in recent years, with an increase in the number and quality of coupled ocean-atmosphere models (Chapter 8) and 


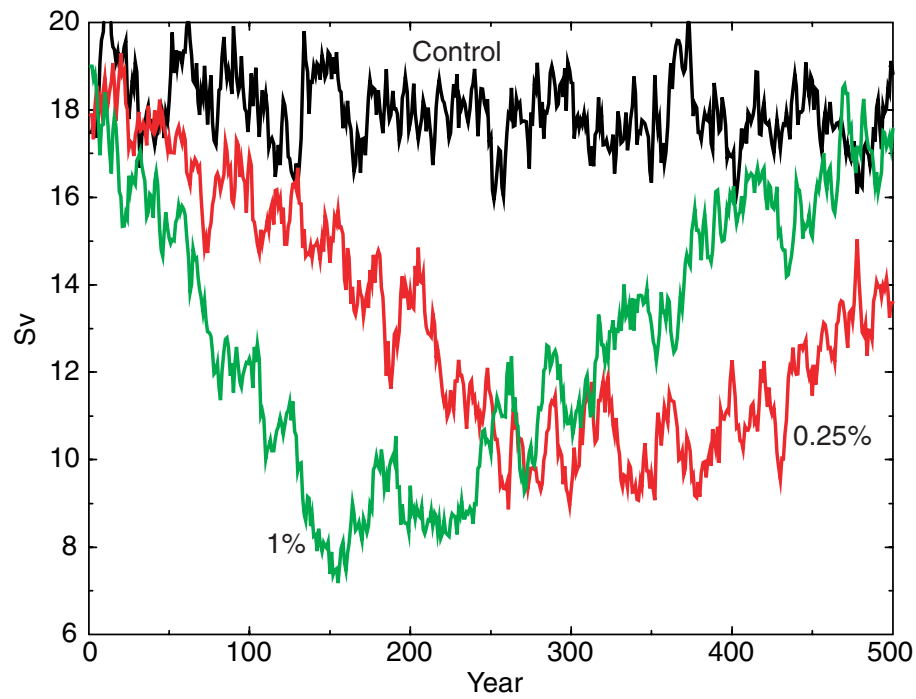

Figure 9.25: Time-series of the maximum value of the stream function $(\mathrm{Sv})$ between $40^{\circ} \mathrm{N}$ and $60^{\circ} \mathrm{N}$ in the Atlantic Ocean for the control (black line), $1 \%$ (green line) and $0.25 \%$ (red line) integrations with the GFDL_R15_a model. See text for integration definitions.

with the running of multi-century experiments and multi-member ensembles of integrations for a given climate forcing (Section 9.2). There have been a number of studies that have considered changes in interannual variability under climate change (e.g., Knutson and Manabe, 1994; Knutson et al., 1997; Tett et al. 1997; Timmermann et al. 1999; Boer et al. 2000b; Collins, 2000a,b). Other studies have looked at intra-seasonal variability in coupled models and the simulation of changes in mid-latitude storm tracks (e.g., Carnell et al. 1996; Lunkeit et al., 1996; Carnell and Senior, 1998; Ulbrich and Christoph, 1999), tropical cyclones (Bengtsson et al., 1996; Henderson-Sellers et al., 1998; Knutson et al., 1998; Krishnamurti et al., 1998; Royer et al., 1998) or blocking anticyclones (Lupo et al., 1997; Zhang and Wang, 1997; Carnell and Senior, 1998). The results from these models must still be treated with caution as they cannot capture the full complexity of these structures, due in part to the coarse resolution in both the atmosphere and oceans of the majority of the models used (Chapter 8).

An expanding area of research since the SAR is the consideration of whether climate change may be realised as preferred modes of non-linear naturally occurring atmospheric circulation patterns, or so-called weather regimes as proposed by Palmer (1999). Recent work (e.g., Hurrell 1995, 1996; Thompson and Wallace 1998; Corti et al., 1999) has suggested that the observed warming over the last few decades may be manifest as a change in frequency of these naturally preferred patterns (Chapters 2 and 7) and there is now considerable interest in testing the ability of climate models to simulate such weather regimes (Chapter 8) and to see whether the greenhouse gas forced runs suggest shifts in the residence time or transitions between such regimes on long time-scales. There are now several multi-ensemble simulations using scenarios of time-evolving forcing and multi-century experiments with stabilised forcing, which may help to separate the noise of decadal variability from the signal of climate change.
In this section, changes in variability (defined as the deviation from some mean value) will be considered on different time-scales (intra-seasonal, interannual, and decadal and longer). Particular attention will be given to changes in naturally occurring modes of variability such as ENSO, the Arctic Oscillation (AO; and its more spatially restricted counterpart, the North Atlantic Oscillation, NAO) and the Antarctic Oscillation (AAO) etc.

\subsubsection{Intra-seasonal variability}

Daily precipitation variability

Changes in daily variability of temperature and rainfall are most obviously manifest in changes in extreme events and much of the work in this area will be discussed in the extreme events section (Section 9.3.6). However, changes in short time-scale variability do not necessarily only imply changes in extreme weather. More subtle changes in daily variability, when integrated over time, could still have important socio-economic impacts. Hennessey et al. (1997) found that the simulated number of wet days (days where the rainfall is non-zero) in two mixed-layer models went down in mid-latitudes and up in high latitudes when $\mathrm{CO}_{2}$ was doubled, whilst the mean precipitation increased in both areas. The global mean precipitation also increased, by around $10 \%$ in both models, typical of the changes in many mixed-layer models on doubling $\mathrm{CO}_{2}$. An analysis of changes in daily precipitation variability in a coupled model (Durman et al., 2001) suggests a similar reduction in wet days over Europe where the increase in precipitation efficiency exceeds the increase in mean precipitation.

\section{Circulation patterns}

Kattenberg et al. (1996) reported research on changes in intermonthly temperatures and precipitation variability from two coupled models (Meehl et al, 1994; Parey, 1994). More recently, there have been several studies looking at changes in intraseasonal circulation patterns using higher resolution atmosphereonly models with projected SSTs taken from coupled models at given time periods in the future (e.g., Beersma et al., 1997; Schubert et al., 1998). The effects of changes in extra-tropical storms on extreme wind and precipitation events are described in Section 9.3.6, but there has also been work on changes in lowerfrequency variability such as persistent or "blocking" anticyclones. As discussed in the SAR, there still seems to be little consensus on the methodology for looking at changes in storms and blocks and it is likely that this is partly the reason for the lack of consistency in results. In new studies, Lupo et al. (1997) looked at the effect of doubled $\mathrm{CO}_{2}$ on several of the characteristics of blocking. They found an increase in the number of continental blocks and a general increase in the persistence of blocks, but with weakened amplitude. In contrast, Carnell and Senior (1998) found the largest change was a decrease in blocking in the North Pacific Ocean in winter in their model. Earlier studies have pointed to the possible model dependency of results (Bates and Meehl, 1986) and Carnell and Senior (2000) suggest that the changes in blocking found in their earlier study (Carnell and Senior, 1998) may depend on the meridional gradient of temperature change in the model, which may in turn depend on the simulation of cloud feedback in their model. 
Zhang and Wang (1997) found a decrease in the total number of Northern Hemisphere winter anticyclones under increased greenhouse gases, although they did not specifically look at blocking anticyclones.

Fyfe (1999) has looked at changes in African easterly waves due to a doubling of $\mathrm{CO}_{2}$ in one model. Significant low-level warming and increases in atmospheric humidity over the Northern Sahara lead to an increase in the easterly wave activity. Again, these results must be considered speculative given the relatively low resolution of the model (T32, about $3.5^{\circ}$ resolution), which leads to substantial systematic biases in the present day simulation of the low-level storm track in the region.

\subsubsection{Interannual variability}

ENSO

ENSO is associated with some of the most pronounced year-toyear variability in climate features in many parts of the world (Chapters 2 and 7). Since global climate models simulate some aspects of ENSO-like phenomena (Chapter 8), there have been a number of studies that have attempted to use climate models to assess the changes that might occur in ENSO in connection with future climate warming and in particular, those aspects of ENSO that may affect future climate extremes.

Firstly, will the long-term mean Pacific SSTs shift toward a more El Niño-like or La Niña-like regime? Since 1995, the analyses of several global climate models indicate that as global temperatures increase due to increased greenhouse gases, the Pacific climate will tend to resemble a more El Niño-like state (Knutson and Manabe, 1995; Mitchell et al., 1995; Meehl and Washington, 1996; Timmermann et al., 1999; Boer et al., 2000b). However, the reasons for such a response are varied, and could depend on the model representation of cloud feedback (Senior, 1999; Meehl et al., 2000b); the quality of the unperturbed El Niño state in the models (Chapter 8) or the stronger evaporative damping of the warming in the warm pool region, relative to the eastern Pacific due to the non-linear Clausius-Clapeyron relationship between temperature and saturation mixing ratios (e.g., Knutson and Manabe, 1995). Additionally, a different coupled model (Noda et al., 1999b) shows a La Niña-like response and yet another model shows an initial La Niña-like pattern which becomes an El Niño-like pattern due to subducted warmed extra-tropical water that penetrates through the sub-tropics into the tropics (Cai and Whetton, 2000). A possible reason for the La Niña-like response has been suggested in a simple coupled model study where the dominant role of ocean dynamics in the heat balance over the tropical Pacific is seen for a specified uniform positive forcing across the Pacific basin (Cane et al., 1997).

Secondly, will El Niño variability (the amplitude and/or the frequency of temperature swings in the equatorial Pacific) increase or decrease? Attempts to address this question using climate models have again shown conflicting results, varying from slight decreases or little change in amplitude (Tett 1995; Knutson et al., 1997; Noda et al., 1999b; Collins, 2000b; Washington et al., 2001; Figure 9.26b) to a small increase in amplitude (Timmermann et al., 1999; Collins, 2000a; Figure 9.26a), which has been attributed to an increase in the intensity of
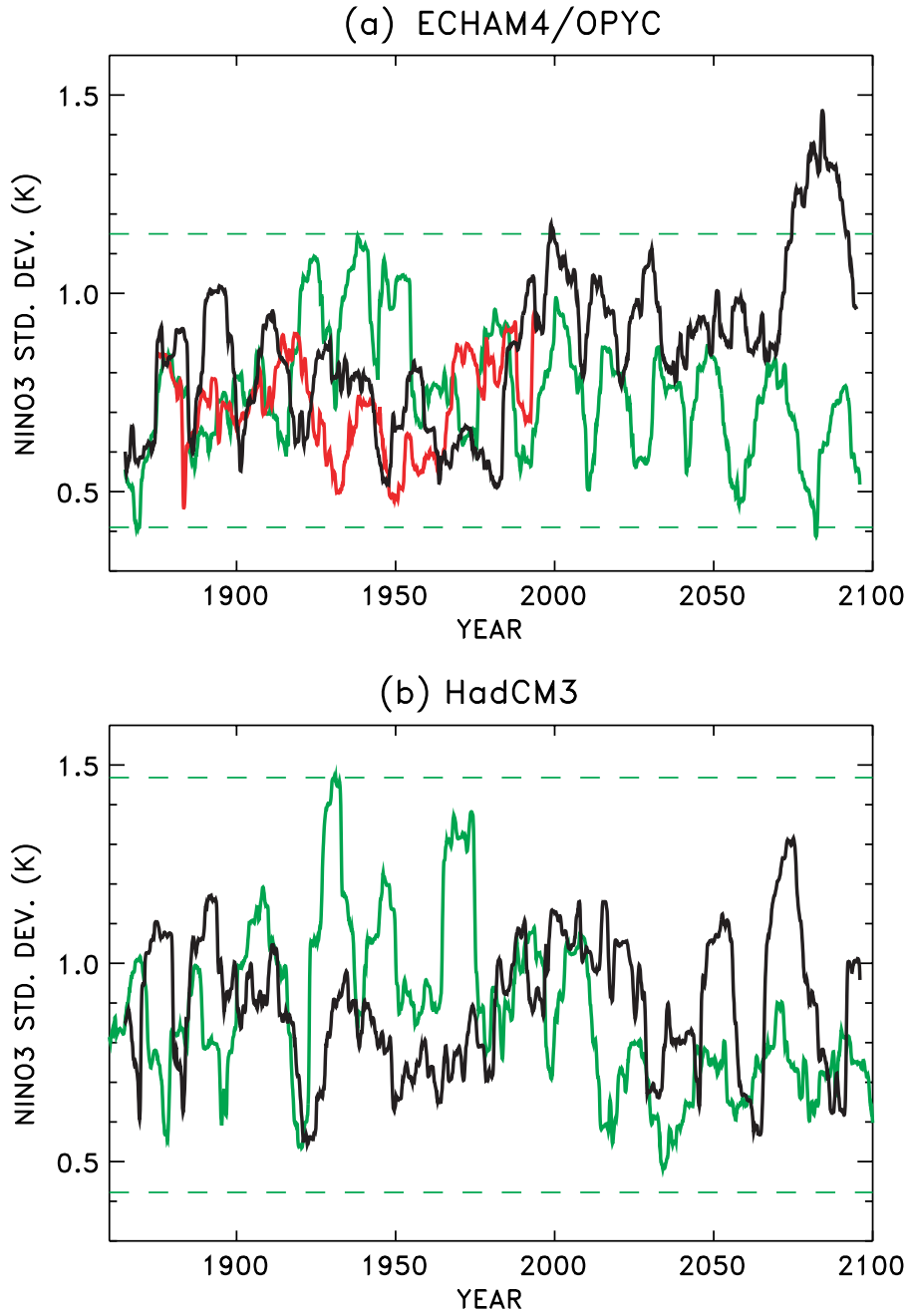

Figure 9.26: Standard deviations of Niño-3 SST anomalies (Unit: ${ }^{\circ} \mathrm{C}$ ) as a function of time during transient greenhouse warming simulations (black line) from 1860 to 2100 and for the same period of the control run (green line). Minimum and maximum standard deviations derived from the control run are denoted by the dashed green lines. A low-pass filter in the form of a sliding window of 10 years width was used to compute the standard deviations. (a) ECHAM4/OPYC model. Also shown is the time evolution of the standard deviation of the observed from 1860 to 1990 (red line). Both the simulated and observed SST anomalies exhibit trends towards stronger interannual variability, with pronounced inter-decadal variability superimposed, (reproduced from Timmermann et al., 1999), (b) HadCM3 (Collins, 2000b).

the thermocline in the tropical Pacific. Knutson et al. (1997) and Hu et al. (2001) find that the largest changes in the amplitude of ENSO occur on decadal time-scales with increased multi-decadal modulation of the ENSO amplitude. Several authors have also found changes in other statistics of variability related to ENSO. Timmermann et al. (1999) find that the interannual variability of their model becomes more skewed towards strong cold (La Niña type) events relative to the warmer mean climate. Collins (2000a) finds an increased frequency of ENSO events and a shift in the seasonal cycle, so that the maximum occurs between August and October rather than around January as in the unperturbed model and the observations. Some recent coupled models have achieved 
a stable climate without the use of flux adjustments and an important question to ask is what is the effect of flux adjustment on changes in variability. Collins (2000b) finds different responses in ENSO in two models, one of which has been run without the use of flux-adjustments. However, he concludes that differences in response are most likely to be due to differences in the response of the meridional temperature gradient in the two models arising from different cloud feedbacks (Williams et al., 2001) rather than due to the presence or absence of flux adjustment.

Finally, how will ENSO's impact on weather in the Pacific Basin and other parts of the world change? Meehl et al. (1993) and Meehl and Washington (1996) indicate that future seasonal precipitation extremes associated with a given ENSO event are likely to be more intense due to the warmer, more El Niño-like, mean base state in a future climate. That is, for the tropical Pacific and Indian Ocean regions, anomalously wet areas could become wetter and anomalously dry areas become drier during future ENSO events. Also, in association with changes in the extratropical base state in a future warmer climate, the teleconnections to mid-latitudes, particularly over North America, may shift somewhat with an associated shift of precipitation and drought conditions in future ENSO events (Meehl et al., 1993).

When assessing changes in ENSO, it must be recognised that an "El Niño-like" pattern can apparently occur at a variety of time-scales ranging from interannual to inter-decadal (Zhang et al., 1997), either without any change in forcing or as a response to external forcings such as increased $\mathrm{CO}_{2}$ (Meehl and Washington, 1996; Knutson and Manabe, 1998; Noda et al., 1999a,b; Boer et al., 2000b; Meehl et al., 2000b). Making conclusions about "changes" in future ENSO events will be complicated by these factors. Additionally, since substantial internally generated variability of ENSO statistics on multidecadal to century time-scales occurs in long unforced climate model simulations (Knutson et al., 1997), the attribution of past and future changes in ENSO amplitude and frequency to external forcing may be quite difficult, perhaps requiring extensive use of ensemble climate experiments or long experiments with stabilised forcing (e.g., Knutson et al., 1997).

Although there are now better ENSO simulations in global coupled climate models (Chapter 8), further model improvements are needed to simulate a more realistic Pacific climatology and seasonal cycle as well as more realistic ENSO variability (e.g., Noda et al., 1999b). It is likely that such things as increased ocean resolution, atmospheric physics and possibly flux correction can have an important effect on the response of the ENSO in models. Improvements in these areas will be necessary to gain further confidence in climate model projections.

\section{Monsoon}

One of the most significant aspects of regional interannual variability is the Asian Monsoon. Several recent studies (Kitoh et al., 1997; Hu et al., 2000a; Lal et al., 2000) have corroborated earlier results (Mitchell et al., 1990; Kattenberg et al., 1996) of an increase in the interannual variability of daily precipitation in the Asian summer monsoon with increased greenhouse gases. Lal et al. (2000) find that there is also an increase in intra-seasonal precipitation variability and that both intra-seasonal and interannual increases are associated with increased intra-seasonal convective activity during the summer. Less well studied is the Asian winter monsoon, although $\mathrm{Hu}$ et al. (2000b) find reductions in its intensity with a systematic weakening of the north-easterlies along the Pacific coast of the Eurasian continent. However, they find no change in the interannual or inter-decadal variability.

The effect of sulphate aerosols on Indian summer monsoon precipitation is to dampen the strength of the monsoon compared to that seen with greenhouse gases only (Lal et al., 1995; Cubasch et al., 1996; Meehl et al., 1996; Mitchell and Johns 1997; Roeckner et al., 1999), reinforcing preliminary findings in the SAR. The pattern of response to the combined forcing is at least partly dependent on the land-sea distribution of the aerosol forcing, which in turn may depend upon the relative size of the direct and indirect effects (e.g., Meehl et al., 1996; Roeckner et al., 1999). There is still considerable uncertainty in these forcings (Chapter 6). To date, the effect of aerosol forcing (direct and indirect) on the variability of the monsoon has not been investigated.

In summary, an intensification of the Asian summer monsoon and an enhancement of summer monsoon precipitation variability with increased greenhouse gases that was reported in the SAR has been corroborated by new studies. The effect of sulphate aerosols is to weaken the intensification of the mean precipitation found with increases in greenhouse gases, but the magnitude of the change depends on the size and distribution of the forcing.

\subsubsection{Decadal and longer time-scale variability}

A few studies have attempted to look at model-simulated changes in modes of low-frequency variability due to anthropogenic climate change. Particular attention has focused on changes in ENSO as reported in the SAR and in Section 9.3.5.2, and the AO or NAO and AAO which are prominent features of low-frequency variability in the Northern and Southern Hemispheres, respectively (e.g., Fyfe et al., 1999; Osborn et al., 1999; Paeth et al., 1999; Shindell et al., 1999; Ulbrich and Christoph, 1999; Zorita and González-Rouco, 2000; Monahan et al., 2000). It should be noted that these studies have used a variety of methods for analysing trends in these modes of variability, including indices based on pressure differentials and principal components (PCs) of hemispheric sea level pressure (SLP). In addition, these indices are sensitive to changes in the SLP patterns with time or forcing and so trends must be treated with some caution. Wallace (2000) finds that in both observations and modelling studies of increased greenhouse gases, the trends are larger in the PC of SLP than in the pressure differential indices. Meehl et al. (2000c) show that the changed base climate state in a future warmer climate could affect the period of global ENSO-like decadal (10 to 20 -year period) variability such that there could be a shift to longer periods.

Ulbrich and Christoph (1999) find that the NAO index, based on SLP fluctuations over the North Atlantic in the 300-year control run of their model, shows only a moderate increase over the length of a 240-year scenario run with increasing greenhouse gases. The long-term trend exceeds the variability of the control climate only at the end of the simulation in 2100. In contrast, the steadily growing storm track activity over north-west Europe 


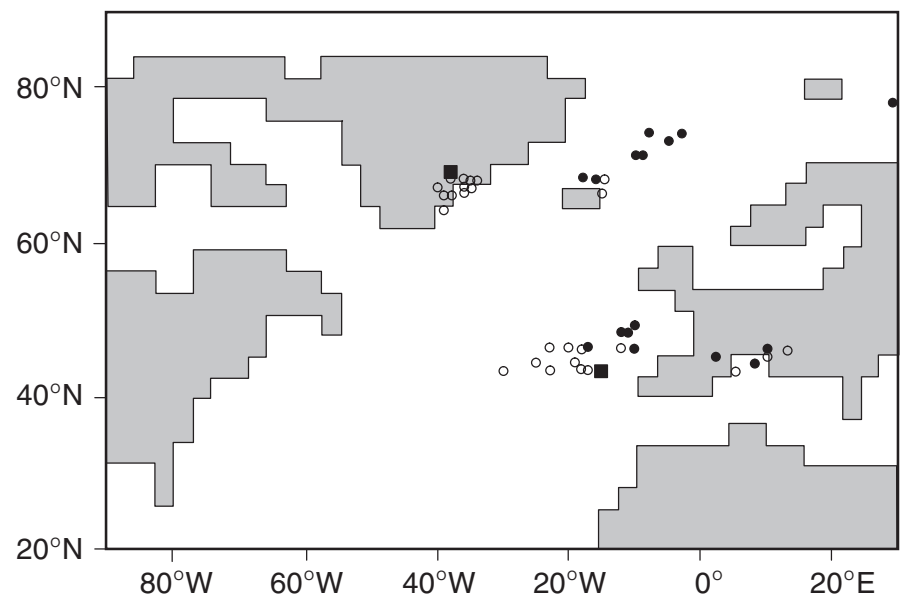

Figure 9.27: Locations of NAO centres (taken to be the position of maximum variance as computed from an EOF analysis of sea level pressure fields) of ECHAM4/OPYC (Ulbrich and Christoph, 1999). The average positions from the entire control run (using winter means) are marked by black squares, those of consecutive decades in the scenario run (using all individual months) are marked by open circles before year 2020 and by black dots thereafter.

already surpasses the standard deviation defined from the control run after about 160 years. This effect is associated with a change of the NAO pattern. During the length of the scenario experiment, empirical orthogonal functions for sequential 10 -year periods show a systematic north-eastward shift of the NAO's northern variability centre from a position close to the east coast of Greenland, where it is also located in the control run, to the Norwegian Sea (Figure 9.27)

Osborn et al. (1999) show an initial small increase followed by a decrease in the NAO index in one model when forced with increases in greenhouse gases or with greenhouse gases and sulphate aerosols. Paeth et al. (1999) have assessed changes in both the mean and variance of the NAO on decadal time-scales at quadrupled $\mathrm{CO}_{2}$-concentrations using an ensemble of four integrations of a single model. They find a statistically significant increase in the mean NAO index (at the 95\% confidence level), especially during late summer/autumn and in winter, suggesting more westerly and typically milder weather over Europe during the cold season. However, the increase in the mean NAO index is accompanied by a reduction in the lowfrequency variability of the NAO (Figure 9.28) (significant at the 5\% significance level after 1910) suggesting that the NAO stabilises in the positive phase. Shindell et al. (1999) found a trend towards more positive values of the $\mathrm{AO}$ index with increased greenhouse gases in a model which included a representation of the stratosphere, but not in troposphere-only versions of the same model. They attribute this to the high correlation of the stratospheric circulation with SLP in the Arctic (e.g., Kitoh et al., 1996; Kodera et al., 1996).

In an ensemble of 1900 to 2100 transient integrations with greenhouse gas and aerosol forcing changes, Fyfe et al. (1999) find a positive trend in the mean $\mathrm{AO}$ and $\mathrm{AAO}$ indices. They argue that in their model this is as a result of essentially

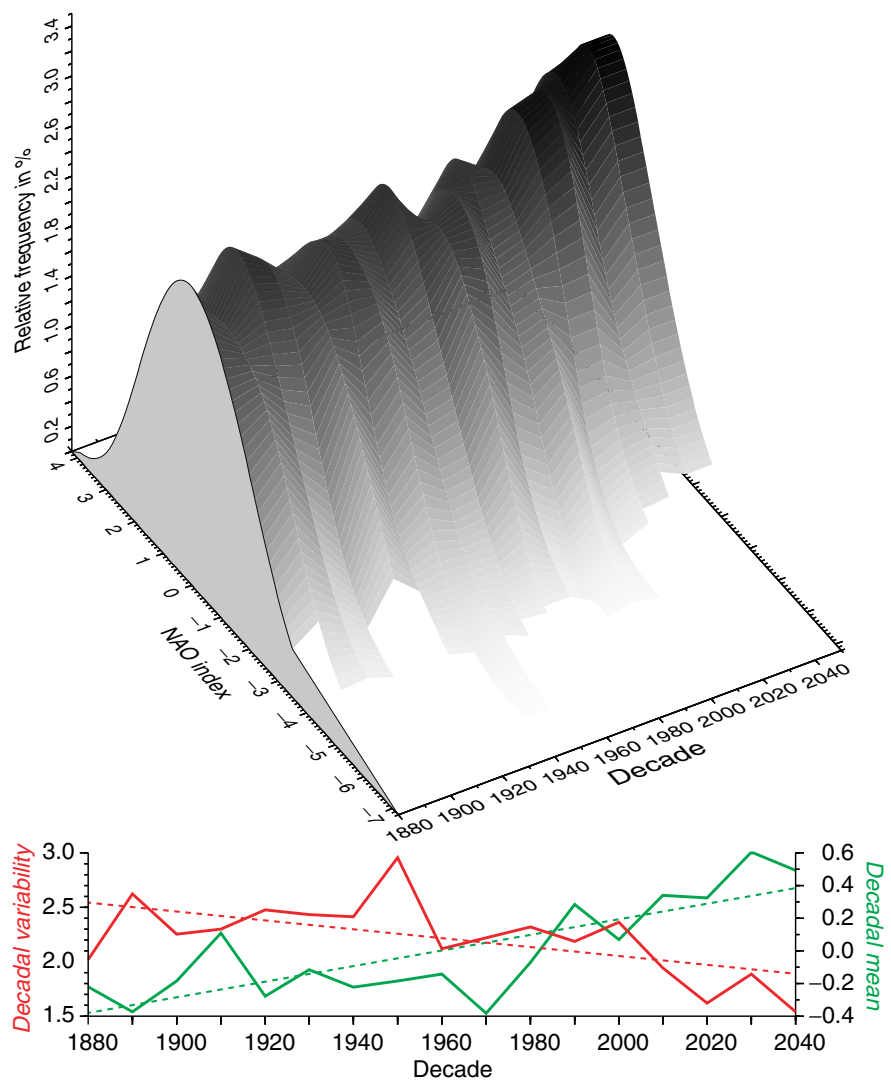

Figure 9.28: Decadal probability density functions (PDF) of the ECHAM3/LSG transient greenhouse gas ensemble: each PDF (greyscale bars) consists of 160 NAO index realisations including the monthly means of November to February of the four simulations over one decade. The greyscale background indicates the relative frequency of the classified NAO indices based on a kernel function (Matyasovszky, 1998). The PDF's width indicates the decadal variability. At the bottom, the time-series of the decadal mean (solid green line) and the variability (solid red line) of each PDF as well as the corresponding linear trends (dashed lines) are shown (from Paeth et al., 1999).

unchanged AO/AAO patterns superimposed onto a forced climate change. The result of Fyfe et al. (1999) suggests that since the mean AO/AAO increases, it might imply a change to higher-frequency variability, as the positive AO phase has enhanced westerlies and is typically correlated with aboveaverage storminess. In a subsequent non-linear analysis by Monahan et al. (2000) of a 1,000-year control and 500-year stabilisation integration (with greenhouse gas and aerosol forcing fixed at their year 2100 levels) it is found that (1) in the control integration the $\mathrm{AO}$ is part of a more general non-linear mode of tropospheric variability which is strongly bimodal and partitions the variability into two distinct regimes, and (2) in the stabilisation integration the occupancy statistics of these regimes change rather than the modes themselves.

In summary, there is not yet a consistent picture emerging from coupled models as to their ability to reproduce trends in climate regimes such as the recently observed upward trend in the NAO/AO index (Chapters 2 and 12). In addition, whilst several 
models show an increase in the NAO/AO index with increased greenhouse gases, this is not true for all models, and the magnitude and character of the changes vary across models. Such results do not necessarily suggest that the forced climate change is manifest as a change in the occurrence of only one phase of these modes of variability.

\subsubsection{Summary}

There are now a greater number of global coupled atmosphereocean models and a number of them have been run for multicentury time-scales. This has substantially improved the basis for estimating long time-scale natural unforced variability. There are still severe limitations in the ability of such models to represent the full complexity of observed variability and the conclusions drawn here about changes in variability must be viewed in the light of these shortcomings (Chapter 8).

Some new studies have reinforced results reported in the SAR. These are:

- The future mean Pacific climate base state could more resemble an El Niño-like state (i.e., a slackened west to east SST gradient with associated eastward shifts of precipitation). Whilst this is shown in several studies, it is not true of all.

- Enhanced interannual variability of daily precipitation in the Asian summer monsoon. The changes in monsoon strength depend on the details of the forcing scenario and model.

Some new results have challenged the conclusions drawn in earlier reports, such as:

- Little change or a decrease in ENSO variability. More recently, increases in ENSO variability have been found in some models where it has been attributed to increases in the strength of the thermocline. Decadal and longer time-scale variability complicates assessment of future changes in individual ENSO event amplitude and frequency. Assessment of such possible changes remains quite difficult. The changes in both the mean and variability of ENSO are still model dependent.

Finally there are areas where there is no clear indication of possible changes or no consensus on model predictions:

- Although many models show an El Niño-like change in the mean state of tropical Pacific SSTs, the cause is uncertain. In some models it has been related to changes in cloud forcing and/or changes in the evaporative damping of the east-west SST gradient, but the result remains model-dependent. For such an El Niño-like climate change, future seasonal precipitation extremes associated with a given ENSO would be more intense due to the warmer mean base state.

- There is still a lack of consistency in the analysis techniques used for studying circulation statistics (such as the AO, NAO and $\mathrm{AAO}$ ) and it is likely that this is part of the reason for the lack of consensus from the models in predictions of changes in such events.
- The possibility that climate change may be expressed as a change in the frequency or structure of naturally occuring modes of low-frequency variability has been raised. If true, this implies that GCMs must be able to simulate such regime transitions to accurately predict the response of the system to climate forcing. This capability has not yet been widely tested in climate models. A few studies have shown increasingly positive trends in the indices of the $\mathrm{NAO} / \mathrm{AO}$ or the $\mathrm{AAO}$ in simulations with increased greenhouse gases, although this is not true in all models, and the magnitude and character of the changes varies across models.

\subsubsection{Changes of Extreme Events}

In this section, possible future changes in extreme weather and climate phenomena or events (discussed in Chapter 2) will be assessed from global models. Regional information derived from global models concerning extremes will be discussed in Chapter 10.

Although the global models have improved over time (Chapter 8), they still have limitations that affect the simulation of extreme events in terms of spatial resolution, simulation errors, and parametrizations that must represent processes that cannot yet be included explicitly in the models, particularly dealing with clouds and precipitation (Meehl et al., 2000d). Yet we have confidence in many of the qualitative aspects of the model simulations since they are able to reproduce reasonably well many of the features of the observed climate system not only in terms of means but also of variability associated with extremes (Chapter 8). Simulations of 20th century climate have shown that including known climate forcings (e.g., greenhouse gases, aerosols, solar) leads to improved simulations of the climate conditions we have already observed. Ensembles of climate change experiments are now being performed to enable us to better quantify changes of extremes.

\subsubsection{Temperature}

Models described in the IPCC First Assessment Report (Mitchell et al., 1990) showed that a warmer mean temperature increases the probability of extreme warm days and decreases the probability of extreme cold days. This result has appeared consistently in a number of more recent different climate model configurations (Dai et al., 2001; Yonetani and Gordon, 2001). There is also a decrease in diurnal temperature range (DTR) since the night-time temperature minima warm faster than the daytime maxima in many locations (e.g., Dai et al., 2001). Although there is some regional variation as noted in Chapter 10 , some of these changes in DTR have also been seen over a number of areas of the world in observations (see Chapter 2). In general, the pattern of change in return values for 20-year extreme temperature events from an equilibrium simulation for doubled $\mathrm{CO}_{2}$ with a global atmospheric model coupled to a nondynamic slab ocean shows moderate increases over oceans and larger increases over land masses (Zwiers and Kharin, 1998; Figure 9.29). This result from a slab ocean configuration without ocean currents is illustrative and could vary from model to model, though it is similar to results from the fully coupled version in a subsequent study (Kharin and Zwiers, 2000). 

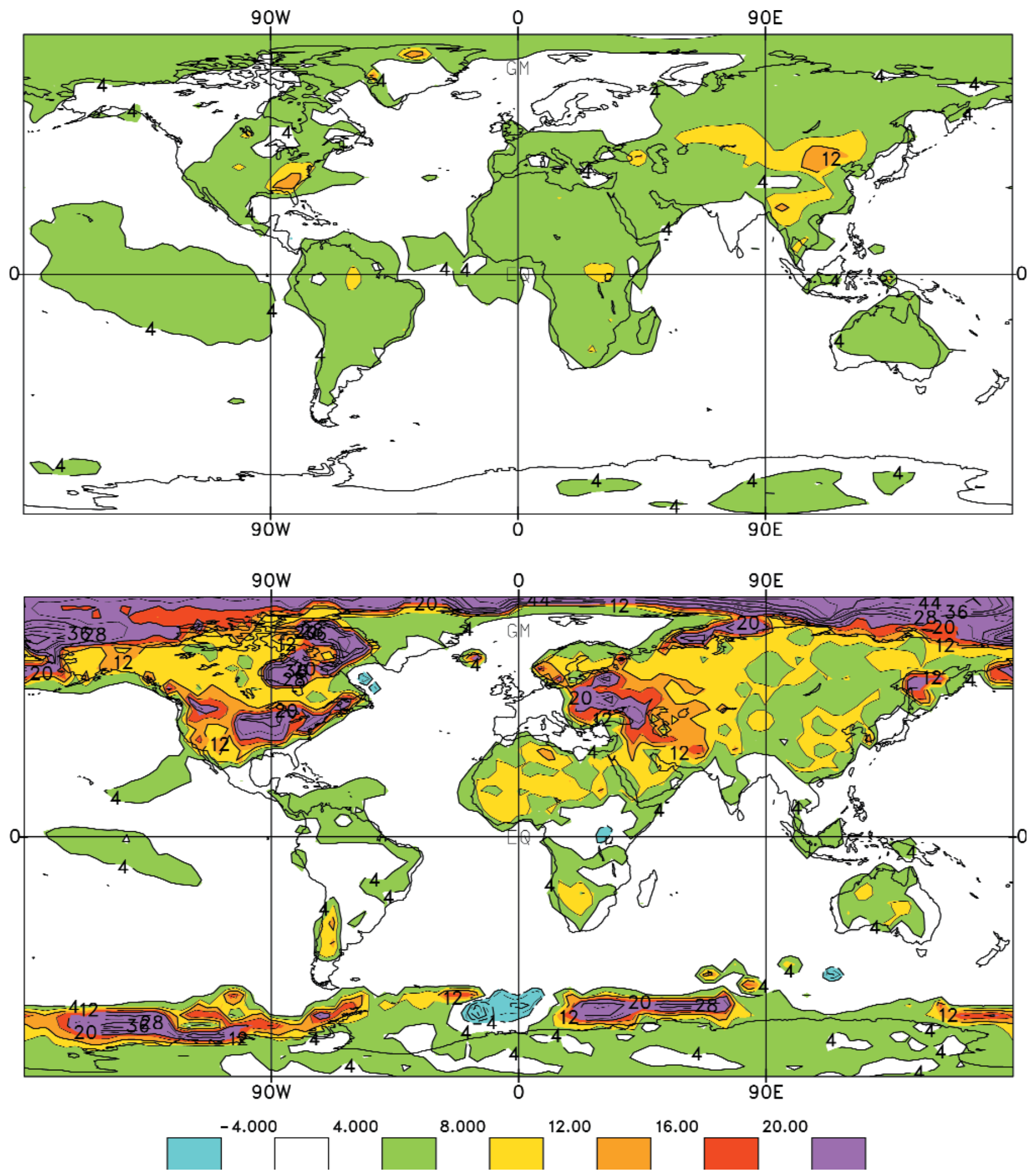

Figure 9.29: The change in 20-year return values for daily maximum (upper panel) and minimum (lower panel) surface air temperature (or screen temperature) simulated in a global coupled atmosphere-ocean model (CGCM1) in 2080 to 2100 relative to the reference period 1975 to 1995 (from Kharin and Zwiers, 2000). Contour interval is $4^{\circ} \mathrm{C}$. Zero line is omitted. 
The greatest increase in the 20-year return values of daily maximum temperature (Figure 9.29, top) is found in central and southeast North America, central and south-east Asia and tropical Africa, where there is a decrease in soil moisture content. Large extreme temperature increases are also seen over the dry surface of North Africa. In contrast, the west coast of North America is affected by increased precipitation resulting in moister soil and more moderate increases in extreme temperature. There are small areas of decrease in the Labrador Sea and Southern Ocean that are associated with changes in ocean temperature. The changes in the return values of daily minimum temperature (Figure 9.29, bottom) are larger than those of daily maximum temperature over land areas and high latitude oceans where snow and ice retreat. Somewhat larger changes are found over land masses and the Arctic while smaller increases in extreme minimum temperatures occur at the margins of the polar oceans. Thus, there is some asymmetry between the change in the extremes of minimum and maximum temperature (with a bigger increase for minima than maxima). This has to do with the change in the nature of the contact between atmosphere and the surface (e.g., minima increase sharply where ice and snow cover have retreated exposing either ocean or land, maxima increase more where the land surface has dried). Consequently there is a seasonal dependence related to changes in underlying surface conditions, which indroduces uncertainties in some regions in some models (Chapter 10).

Simulations suggest that both the mean and standard deviation of temperature are likely to change with a changed climate, and the relative contribution of the mean and standard deviation changes depends on how much each moment changes. Increased temperature variance adds to the probability of extreme high temperature events over and above what could be expected simply from increases in the mean alone. The increased variance of daily temperature in summer in northern mid-continental areas noted above has also been seen in other global models (Gregory and Mitchell, 1995). However, as noted in Chapter 10, such changes can vary from region to region and model to model (e.g., Buishand and Beersma (1996), who showed some small decreases over an area of Europe). The change in the mean is usually larger than the change in variance for most climate change simulations. Climate models have also projected decreased variability of daily temperature in winter over mid-continental Europe (Gregory and Mitchell, 1995). Such a decrease is partly related to a reduction of cold extremes, which are primarily associated with the increased mean of the daily minimum temperature. The detrimental effect of extreme summer heat is likely to be further exacerbated by increased atmospheric moisture. One model scenario shows an increase of about $5^{\circ} \mathrm{C}$ in July mean "heat index" (a measure which includes both the effects of temperature and moisture, leading to changes in the heat index which are larger than changes in temperature alone; it measures effects on human comfort; see further discussion in Chapter 10) over the southeastern USA by the year 2050 (Delworth et al., 2000). Changes in the heating and cooling degree days are another likely extreme temperature-related effect of future greenhouse warming. For example, analysis of these measures shows a decrease in heating degree days for Canada and an increase in cooling degree days in the southwest USA in model simulations of future climate with increased greenhouse gases (Zwiers and Kharin, 1998; Kharin and Zwiers, 2000), though this can be considered a general feature associated with an increase in temperature.

\subsubsection{Precipitation and convection}

Increased intensity of precipitation events in a future climate with increased greenhouse gases was one of the earliest model results regarding precipitation extremes, and remains a consistent result in a number of regions with improved, more detailed models (Hennessy et al., 1997; Kothavala, 1997; Durman et al., 2001; Yonetani and Gordon, 2001). There have been questions regarding the relatively coarse spatial scale resolution in climate models being able to represent essentially mesoscale and smaller precipitation processes. However, the increase in the ability of the atmosphere to hold more moisture, as well as associated increased radiative cooling of the upper troposphere that contributes to destabilisation of the atmosphere in some models, is physically consistent with increases in precipitation and, potentially, with increases in precipitation rate.

As with other changes, it is recognised that changes in precipitation intensity have a geographical dependence. For example, Bhaskharan and Mitchell (1998) note that the range of precipitation intensity over the south Asian monsoon region broadens in a future climate experiment with increased greenhouse gases, with decreases prevalent in the west and increases more widespread in the east (see further discussion in Chapter 10). Another model experiment (Brinkop, 2001) shows that extreme values of the convective rain rate and the maximum convective height occur more frequently during the 2071 to 2080 period than during the 1981 to 1990 period. The frequency of highest-reaching convective events increases, and the same holds for events with low cloud-top heights. In contrast, the frequency of events with moderate-top heights decreases. On days when it rains, the frequency of the daily rates of convective rainfall larger than $40 \mathrm{~mm} /$ day in JJA and greater than $50 \mathrm{~mm} /$ day for DJF, increases. Generally, one finds a strong increase in the rain rate per convective event over most of the land areas on the summer hemispheres and in the inter-tropical convergence zone (ITCZ). Between 10 and $30^{\circ} \mathrm{S}$ there are decreases in rain rate per event over the ocean and parts of the continents.

In global simulations for future climate, the percentage increase in extreme (high) rainfall is greater than the percentage increase in mean rainfall (Kharin and Zwiers, 2000). The return period of extreme precipitation events is shortened almost everywhere (Zwiers and Kharin, 1998). For example, they show that over North America the 20-year return periods are reduced by a factor of 2 indicating that extreme precipitation of that order occurs twice as often.

Another long-standing model result related to drought (a reduction in soil moisture and general drying of the midcontinental areas during summer with increasing $\mathrm{CO}_{2}$ ) has been reproduced with the latest generation of global coupled climate models (Gregory et al., 1997; Haywood et al., 1997; Kothavala, 1999; Wetherald and Manabe, 1999). This summer drying is 
generally ascribed to a combination of increased temperature and potential evaporation not being balanced by precipitation. To address this problem more quantitatively, a global climate model with increased $\mathrm{CO}_{2}$ was analysed to show large increases in frequency of low summer precipitation, the probability of dry soil, and the occurrence of long dry spells (Gregory et al., 1997). The latter was ascribed to the reduction of rainfall events in the model rather than to decreases in mean precipitation. However, the magnitude of this summer drying response may be related to the model's simulation of net solar radiation at the surface, and more accurate simulation of surface fluxes over land will increase confidence in the GCM climate changes.

Alhough of great importance to society for their potential for causing destruction, as well as their human and economic impacts, there is little guidance from AOGCMs concerning the future behaviour of tornadoes, hail or lightning. This is because these phenomena are not explicitly resolved in AOGCMs, and any studies that have been done have had to rely on empirical relationships between model features and the phenomenon of interest. For example, Price and Rind (1994a) derive a relationship between lightning activity and convective cloud-top height to infer an increase of lightning with increasing $\mathrm{CO}_{2}$. They take that relationship one step further to suggest a future increase in lightning-caused fires due to the increased lightning activity and decreased effective precipitation (Price and Rind, 1994b). Using another empirical relationship between daily minimum temperature and severe convective storm frequency for France, Dessens (1995) connects an increase in daily minimum temperature with greater convective storm frequency and more hail damage in a future climate with increased $\mathrm{CO}_{2}$. However, there have been no recent studies examining this problem with the current generation of global climate models. Due to the fact that these severe weather phenomena are sub-grid scale (even more so than discussed below for tropical cyclones), and that second and third order linkages between model output and empirical relationships for limited regions must be used to derive results, we cannot reach any definitive conclusions concerning possible future increases in hail and lightning, and there is no information from AOGCMs concerning future changes in tornado activity.

\subsubsection{Extra-tropical storms}

Storms not only have obvious effects on extremes of temperature and precipitation, but also have severe impacts associated with wind, ocean waves, etc. Due to model limitations in previous generations of global climate models, until recently there have been few studies examining changes in extra-tropical cyclones in a future climate. With the improved recent generation of global climate models (see Chapter 8), such studies are now becoming more credible. An analysis of an ensemble of four future climate change experiments using a global coupled model with increased $\mathrm{CO}_{2}$ and sulphate aerosols showed an increase in the number of deep low pressure systems in Northern Hemisphere winter, while the number of weaker storms was reduced (Carnell and Senior, 1998). Studies using different models show a similar change for both hemispheres (Sinclair and Watterson, 1999) or for a study region limited to the North Atlantic (Knippertz et al., 2000).

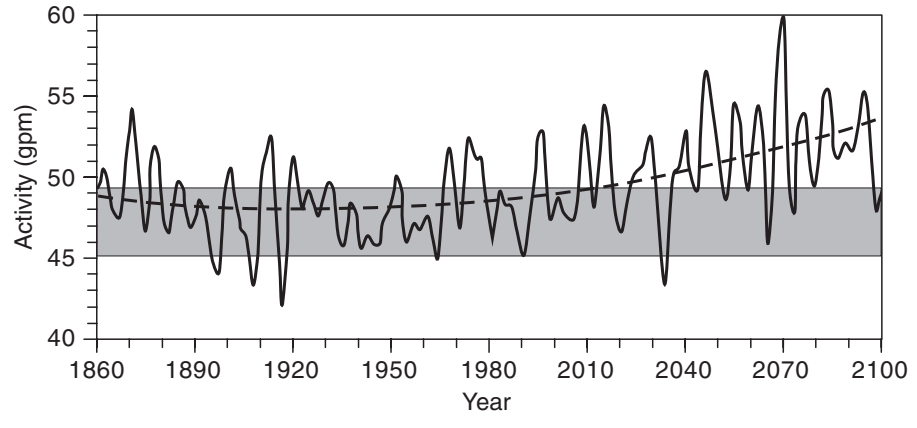

Figure 9.30: Storm track activity averaged over north-west Europe $\left(6^{\circ} \mathrm{W}\right.$ to $20^{\circ} \mathrm{E}, 40^{\circ}$ to $70^{\circ} \mathrm{N}$ ) in the ECHAM4/OPYC greenhouse gas scenario run (Unit: gpm). A 4-year running mean is shown for smoother display. The grey band indicates the variability of this index in the control run as measured by one standard deviation. The non-linear climate trend optimally obtained from quadratic curve fitting is marked by the dashed line; y-axis is activity in gpm (geopotential metres) and $\mathrm{x}$-axis is time in calendar years. From Ulbrich and Christoph (1999).

The reasons given for this common result are still under discussion. Carnell and Senior (1998) ascribe it to a decrease in the mean meridional temperature gradient in the future climate, with high latitudes warming more than low latitudes (producing fewer storms), and greater latent heating in the moister atmosphere (resulting in deeper lows). Sinclair and Watterson (1999) point to the reduced mean sea level pressure and emphasise that vorticity as a measure of cyclone strength does not increase. Knippertz et al. (2000) consider the increasing upper tropospheric baroclinicity to be an important indicator of the change in surface cyclone activity. They also detect an increasing number of strong wind events in their simulation that can be assigned to the increasing number of deep lows. Upper air storm track activity (defined as the standard deviation of the band pass filtered $500 \mathrm{hPa}$ height and related to the surface lows) has been found to increase over the East Atlantic and Western Europe with rising greenhouse gas forcing (such as seen in Figure 9.30 from Ulbrich and Christoph, 1999).

They related this increase to a change in the NAO (see discussion of possible NAO changes in Section 9.3.5.3). Several studies have tried to look at mechanisms of changes (e.g., Lunkeit et al., 1998). For example, Christoph et al. (1997) identify a midwinter suppression of the North Pacific storm track in present day climate which they attribute to very strong upper level winds at that time of year. In a $3 \times \mathrm{CO}_{2}$ climate model experiment, they note that very intense upper level winds occur more often, thus producing a more pronounced mid-winter suppression of the Pacific storm track.

Longer time-series from models have made the statistics more robust (e.g., Carnell and Senior, 1998). High-resolution models may improve the representation of storms, but the present experiments are mainly too short to provide indications of significant changes (e.g., Beersma et al., 1997). As can be seen, there are now a growing number of studies addressing possible changes in storm activity, but in spite of an emerging common signal there remains uncertainty with respect to the governing mechanisms. 


\subsubsection{Tropical cyclones}

Here we assess only AOGCM-related results pertaining to tropical cyclones. For further discussion of results from embedded and mesoscale models regarding possible future changes in tropical cyclone activity, see Chapter 10 (also refer to Box 10.2 for a summary). The ability of global models to accurately represent tropical cyclone phenomena, and their present limitations in this regard, is important for understanding their projection of possible future changes. These capabilities are discussed in detail in Chapter 8.

Some of the global climate models suggest an increase in tropical storm intensities with $\mathrm{CO}_{2}$-induced warming (Krishnamurti et al., 1998), though a limitation of that study is the short two year model run. However, the highest resolution global climate model experiment reported to date (Bengtsson et al., 1996; see Chapter 10) still has a resolution too coarse (about $1^{\circ}$ ) to simulate the most intense storms or realistically simulate structures such as the hurricane eye.

Indices of tropical cyclone activity (Gray, 1979) summarise the necessary large-scale conditions for tropical cyclone activity from coarse resolution GCMs (Evans and Kempisty, 1998; Royer et al., 1998). The latter study examined large-scale atmospheric and oceanic conditions (vertical shear, vorticity and thermodynamic stability), and suggested that only small changes in the tropical cyclone frequencies would occur (up to a $10 \%$ increase in the Northern Hemisphere primarily in the north-west Pacific, and up to a 5\% decrease in the Southern Hemisphere). Climate change studies to date show a great sensitivity to the measure of convective activity chosen, and depend less on the model produced fields. Additionally, the broad geographic regions of cyclogenesis, and therefore also the regions affected by tropical cyclones, are not expected to change significantly (HendersonSellers et al., 1998). This is because results from Holland's (1997) Maximum Potential Intensity model show that even with substantial (1 to $2^{\circ} \mathrm{C}$ ) SST increases in the tropics from global warming, one would also get a correspondingly much bigger warming in the upper troposphere leading to very little change in the moist static stability (Holland, 1997). Another study shows areas of deep convection that can be associated with tropical cyclone formation would not expand with increases in $\mathrm{CO}_{2}$ due to an increase of the SST threshold for occurrence of deep convection (Dutton et al., 2000). Additionally, since tropical storm activity in most basins is modulated by El Niño/La Niña conditions in the tropical Pacific, projections of future regional changes in tropical storm frequencies may depend on accurate projections of future El Niño conditions, an area of considerable uncertainty for climate models (as noted in Section 9.3.5.2).

\subsubsection{Commentary on changes in extremes of weather and climate}

Although changes in weather and climate extremes are important to society, ecosystems, and wildlife, it is only recently that evidence for changes we have observed to date has been able to be compared to similar changes that we see in model simulations for future climate (generally taken to be the end of the $21 \mathrm{st}$ century as shown in this chapter). Though several simulations of 20th century climate with various estimates of observed forcings now exist (see Chapter 8), few of these have been analysed for changes in extremes over the 20th century. So far, virtually all studies of simulated changes in extremes have been performed for future climate. A number of studies are now under way for simulated 20th century climate, but are not yet available for assessment. Additionally, in the 20th century climate integrations there is usually a significant signal/noise problem (especially for changes in phenomena like storms). Therefore, here we assess changes in extremes that have been observed during the 20th century (see Chapter 2), and compare these to simulated changes of extremes for the end of the 21st century from AOGCMs run with increases in greenhouse gases and other constituents. Agreement between the observations and model results would suggest that the changes in extremes we have already observed are qualitatively consistent in a very general way with those changes in climate model simulations of future climate, indicating these changes in extremes would be likely to continue into the future.

The assessment of extremes here relies on very large-scale changes that are physically plausible or representative of changes over many areas. There are some regions where the changes of certain extremes may not agree with the larger-scale changes (see Chapters 2 and 10). Therefore, the assessment here is a general one where observed and model changes appear to be representative and physically consistent with a majority of changes globally. Additionally, certain changes in observed extremes may not have been specifically itemised from model simulations, but are physically consistent with changes of related extremes in the future climate experiments and are denoted as such. Also note that the information for tropical cyclones is drawn from Chapter 10, and diurnal temperature range from Chapter 12. A further discussion of the synthesis of observed and modelled changes of extremes, along with results on how extremes can affect human society, ecosystems and wildlife, appears in Easterling et al. (2000).

The qualitative consistency between the observations from the latter half of the 20th century and the models for the end of the 21st century in Table 9.6 suggests that at least some of the changes we have observed to date are likely to be associated with changes in forcing we have already experienced over the 20th century. The implication is that these could continue to increase into the 21st century with the ongoing rise in forcing from ever greater amounts of greenhouse gases in the atmosphere.

Table 9.6 depicts an assessment of confidence in observed changes in extremes of weather and climate during the latter half of the 20th century (left column) and in projected changes during the 21 st century (right column). As noted above, this assessment relies on observational and modelling studies, as well as the physical plausibility of future projections across all commonly used scenarios and is based on expert judgement. For more details, see Chapter 2 (observations) and Chapter 10 (regional projections).

For the projected changes in the right-hand column, "very likely" indicates that a number of models have been analysed for such a change, all those analysed show it in most regions, and it is physically plausible. No models have been analysed to show fewer frost days, but it is physically plausible, since most models 
Table 9.6: Estimates of confidence in observed and projected changes in extreme weather and climate events.

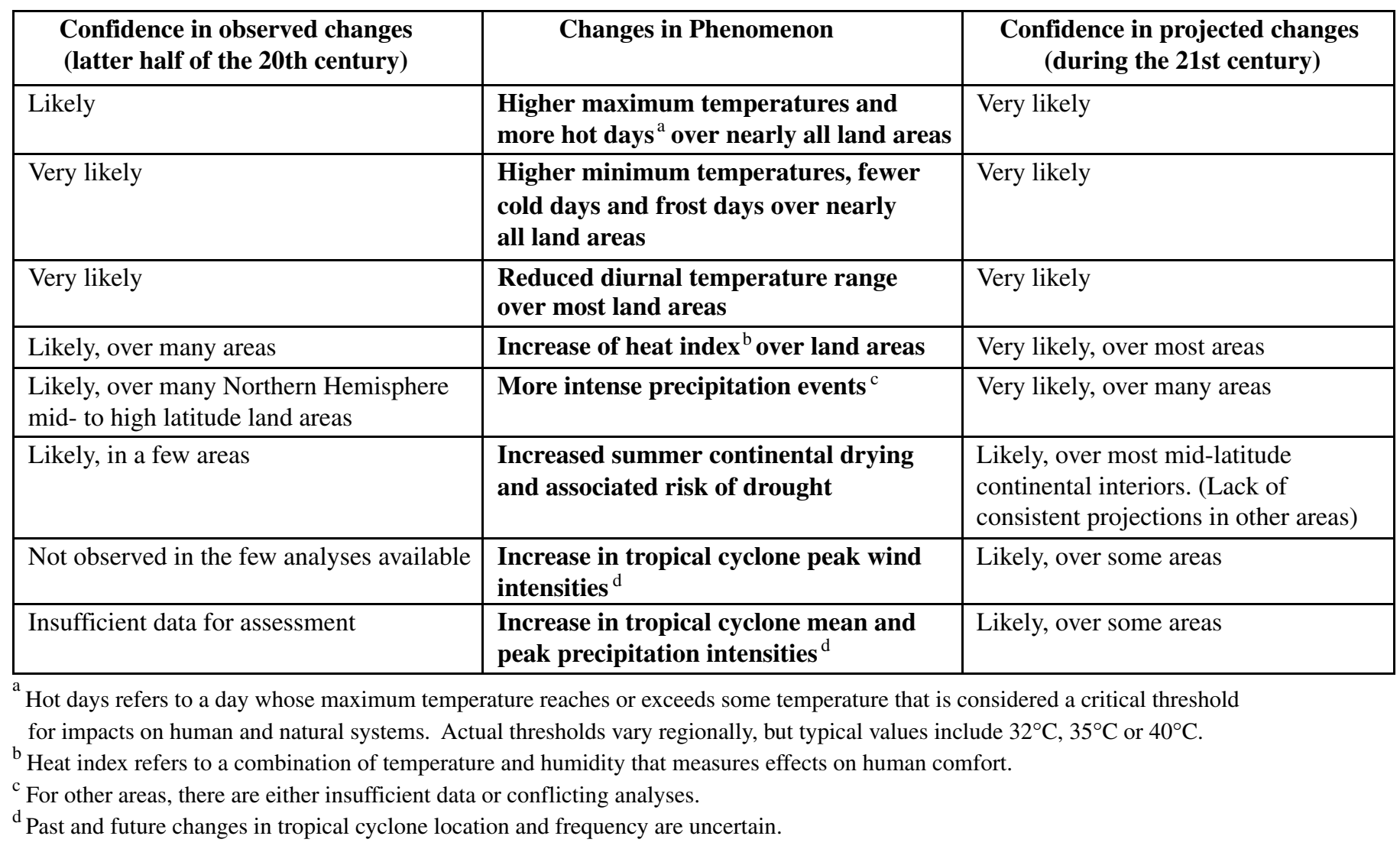

show an increase in night-time minimum temperatures, which would result in fewer frost days. The category "likely" indicates that theoretical studies and those models analysed show such a change, but only a few current climate models are configured in such a way as to reasonably represent such changes. "Hot days" refers to a day whose maximum temperature reaches or exceeds some temperature that is considered a critical threshold for impacts on human and natural systems. Actual thresholds vary regionally, but typical values include $32^{\circ} \mathrm{C}, 35^{\circ} \mathrm{C}$ or $40^{\circ} \mathrm{C}$.

For some other extreme phenomena, many of which may have important impacts on the environment and society, there is currently insufficient information to assess recent trends, and climate models currently lack the spatial detail required to make confident projections. For example, very small-scale phenomena, such as thunderstorms, tornadoes, hail and lightning, are not simulated in climate models at present.

\subsubsection{Conclusions}

Much of what climate model studies show could happen to weather and climate extremes in a future climate with increased greenhouse gases is what we would intuitively expect from our understanding of how the climate system works. For example, a warming of the surface supplies more water vapour to the atmosphere, which is a greater source of moisture in storms and thus we would expect an increase in intense precipitation and more rainfall from a given rainfall event, both results seen in climate model simulations. There are competing effects of decreased baroclinicity in some regions due to greater surface warming at high latitudes, and increasing mid-tropospheric baroclinicity due to greater mid-tropospheric low latitude warming (Kushner et al., 2001). Additionally, a number of changes in weather and climate extremes from climate models have been seen in observations in various parts of the world (decreased diurnal temperature range, warmer mean temperatures associated with increased extreme warm days and decreased extreme cold days, increased rainfall intensity, etc.). Though the climate models can simulate many aspects of climate variability and extremes, they are still characterised by systematic simulation errors and limitations in accurately simulating regional climate such that appropriate caveats must accompany any discussion of future changes in weather and climate extremes.

Recent studies have reproduced previous results in the SAR and this gives us increased confidence in their credibility (although agreement between models does not guarantee that those changes will occur in the real climate system):

- An increase in mean temperatures leads to more frequent extreme high temperatures and less frequent extreme low temperatures.

- Night-time low temperatures in many regions increase more than daytime highs, thus reducing the diurnal temperature range. 
- Decreased daily variability of temperature in winter and increased variability in summer in Northern Hemisphere midlatitude areas.

- There is a general drying of the mid-continental areas during summer in terms of decreases in soil moisture, and this is ascribed to a combination of increased temperature and potential evaporation not being balanced by precipitation.

- Intensity of precipitation events increases.

Additional results since 1995 include:

- Changes in temperature extremes noted above have been related to an increase in a heat index (leading to increased discomfort and stress on the human body), an increase in cooling degree days and a decrease in heating degree days.

- Additional statistics relating to extremes are now being produced. For example, in one model the greatest increase in the 20-year return values of daily maximum temperature is found in central and Southeast North America, central and Southeast Asia and tropical Africa where there is a decrease in soil moisture content, and also over the dry surface of North Africa. The west coast of North America is affected by increased precipitation, resulting in moister soil and more moderate increases in extreme temperature. The increases in the return values of daily minimum temperature are larger than those of daily maximum temperature mainly over land areas and where snow and sea ice retreat.

- Precipitation extremes increase more than the mean and that means a decrease in return period for the extreme precipitation events almost everywhere (e.g., 20 to 10 years over North America).

Aspects which have been addressed but remain unresolved at this time include:

- There is no general agreement yet among models concerning future changes in mid-latitude storms (intensity, frequency and variability), though there are now a number of studies that have looked at such possible changes and some show fewer weak but greater numbers of deeper mid-latitude lows, meaning a reduced total number of cyclones.

- Due to the limitations of spatial resolution in current AOGCMs, climate models do not provide any direct information at present regarding lightning, hail, and tornadoes. Results derived from earlier models used empirical relationships to infer a possible future increase in lightning and hail, though there have been no recent studies to corroborate those results.

- There is some evidence that shows only small changes in the frequency of tropical cyclones derived from large-scale parameters related to tropical cyclone genesis, though some measures of intensities show increases, and some theoretical and modelling studies suggest that upper limit intensities could increase.

\subsection{General Summary}

Figure 9.31 summarises some of the model results for projections of future climate change for the end of the 21 st century. This figure can be compared to one for observations from the 20th century in Chapter 2 (Figure 2.37). A number of the observed changes are qualitatively consistent with those projected for future climate changes from climate models. A confidence scale is provided for the model projections in Figure 9.31, and is the same as the one used in the Executive Summary. Since there is considerable agreement between the observations in Figure 2.37 and the model results listed in Figure 9.31, we conclude that many of the larger observed climate changes to date are qualitatively consistent with those changes in climate models for future climate with increases of greenhouse gases.

(a) Temperature indicators

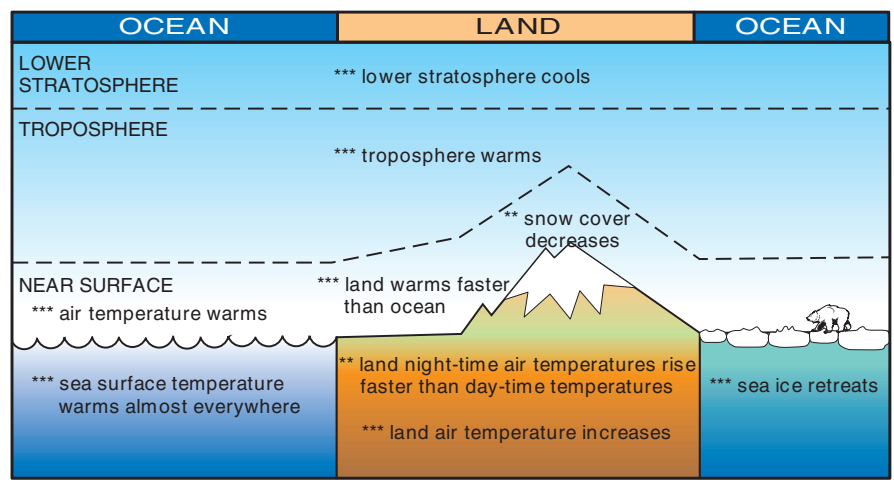

(b) Hydrological and storm-related indicators

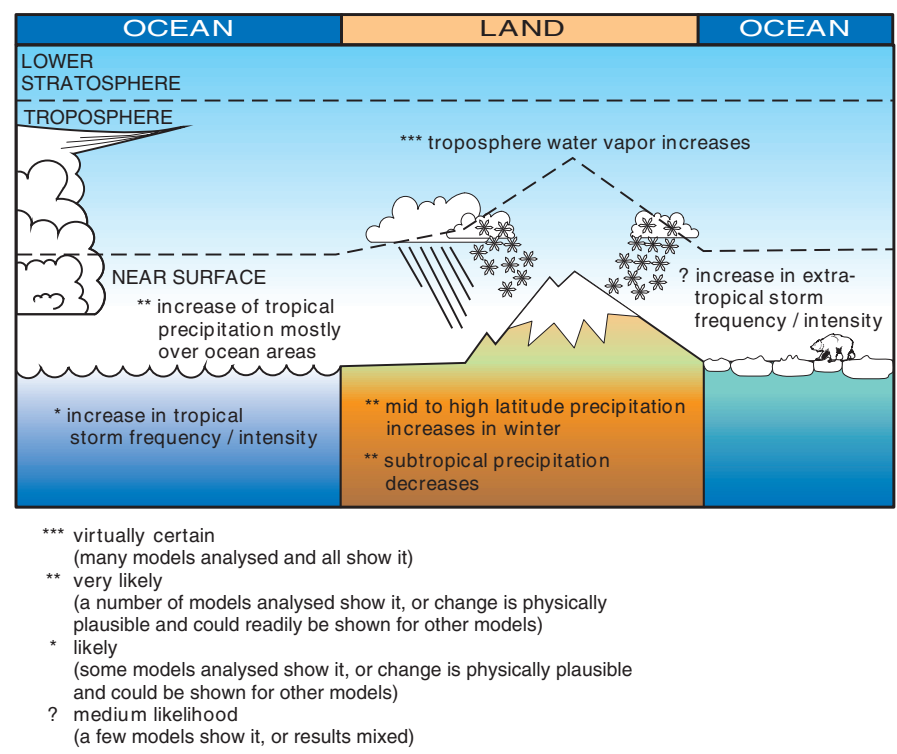

Figure 9.31: Schematic of changes in the temperature and hydrological indicators from projections of future climate changes with AOGCMs. This figure can be compared with Figure 2.37 to note climate changes already observed, to provide a measure of qualitative consistency with what is projected from climate models. 


\section{Appendix 9.1: Tuning of a Simple Climate Model to AOGCM Results}

The simple climate model MAGICC (Wigley and Raper; 1987, 1992; updated in Raper et al., 1996) was used in the SAR to make temperature projections for various forcing scenarios and for sensitivity analyses. The justification for using the simple model for this purpose was the model's ability to simulate AOGCM results in controlled comparisons spanning a wide range of forcing cases (for example SAR Figure 6.13). The approach used in this report differs from that in the SAR. Thus the upwelling diffusion-energy balance model (UD/EB) model is not used here as a stand-alone model in its own right but instead it is tuned to individual AOGCMs and is used only as a tool to emulate and extend their results. In this way, a range of results is produced reflecting the range of AOGCM results. The tuning is based on the CMIP2 data analysis of Raper et al. (2001b). The validity of the tuning is tested by comparisons with AOGCM results in the DDC data set and, where available, with recent AOGCM results using the SRES scenarios. By using such simple models, differences between different scenarios can easily be seen without the obscuring effects of natural variability, or the similar variability that occurs in coupled AOGCMs (Harvey et al., 1997). Simple models also allow the effect of uncertainties in the climate sensitivity and the ocean heat uptake to be quantified. Potentially, other simple models (for example, Watterson (2000), Visser et al. (2000)) could be used in a similar way.

The first step in the tuning process is to select appropriate values for the radiative forcing for a $\mathrm{CO}_{2}$ doubling parameter, $F_{2 x}$, and the climate sensitivity parameter, $T_{2 x}$. In the SAR, $F_{2 \mathrm{x}}=4.37$ $\mathrm{Wm}^{-2}$ was used, as given in the 1990 IPCC Assessment (Shine et al., 1990). This value, which did not account for stratospheric adjustment and solar absorption by $\mathrm{CO}_{2}$, is now considered to be too high (Myhre et al., 1998). These authors suggest a best estimate of $3.71 \mathrm{Wm}^{-2}$; model-specific values are used here (see Table 9.A1). The effect on global mean temperature and sea level change of using lower values of $F_{2 x}$ has been investigated by Wigley and Smith (1998). The lower $F_{2 x}$ values result in slightly lower temperature projections. Different definitions and methods of calculation of model climate sensitivity are discussed in Section 9.3.4.1. Here the effective climate sensitivities based on the last twenty years of the CMIP2 data are used.

Having selected the value of $F_{2 x}$ and $T_{2 x}$ appropriate to a specific AOGCM, the simple model tuning process consists of matching the AOGCM net heat flux across the ocean surface by adjusting the simple model ocean parameters following Raper $e t$ al. (2001a), using the CMIP2 results analysed in Raper et al. (2001b). Sokolov and Stone (1998) show that when using a pure diffusion model to match the behaviour of different AOGCMs a wide range of diffusion coefficients is needed. The range here is much smaller because a 1-D upwelling diffusion model is used and changes in the strength of the thermohaline circulation are also accounted for. A decrease in the strength of the thermohaline circulation leads to an increased heat flux into the ocean. In the $\mathrm{UD/EB}$ model a weakening of the thermohaline circulation is represented by a decline in the upwelling rate (see SAR). The rate of sea level rise from thermal expansion for a collapse in the thermohaline circulation in the UD/EB model is tuned to match that which occurs for an induced collapse in the GFDL model (GFDL_R15_a) control run. An instantaneous 30\% decline in the UD/EB model upwelling rate gives rates of sea level rise comparable to that seen in the GFDL model over a period of 500 years. Thus a 30\% decline in the UD/EB model upwelling rate represents a collapse in the thermohaline circulation. For the individual models the rate of decline in the strength of the thermohaline circulation relative to the global mean temperature change is based on the CIMP2 data and is specified by the parameter $\Delta \mathrm{T}^{+}$. It should be pointed out that the processes in the UD/EB model that determine the heat flux into the ocean are not necessarily physically realistic. Raper and Cubasch (1996) as well as Raper $e t$ al. (2001a) show that the net heat flux into the ocean in the UD/EB model can be tuned to match that in an AOGCM in several ways, using different sets of parameter values. Nevertheless, if the UD/EB model is carefully tuned to match the results of an AOGCM, and provided the extrapolations are not too far removed from the results used for tuning, the UD/EB model can be used to give reasonably reliable estimates of AOGCM temperature changes for different forcing scenarios. The thermal expansion results are less reliably reproduced because thermal expansion is related to the integrated heat flux into the ocean. Errors therefore tend to accumulate. In addition, the expansion depends on the distribution of warming in the ocean. Nonetheless, the simulation is adequate for comparison of scenarios.

Other parameters in the UD/EB model are adjusted in order to correctly simulate the greater surface temperature change over the land relative to the ocean as shown to a varying degree in different AOGCM results. The land-ocean, Northern-Southern Hemisphere temperature change contrasts are adjusted by parameters that govern the contrast in the land-ocean climate sensitivity and the land-ocean exchange coefficients. The specific parameter values used for the different AOGCMs are given in Table 9.A1.

Table 9.A1: Simple climate model parameter values used to simulate AOGCM results. In all cases the mixed-layer depth $\mathrm{hm}=60 \mathrm{~m}$, the sea ice parameter CICE $=1.25$ and the proportion of the upwelling that is scaled for a collapse of the thermohaline circulation is 0.3 , otherwise parameters are as used in the SAR (Kattenberg et al., 1996; Raper et al., 1996).

\begin{tabular}{|l|c|c|c|c|c|c|}
\hline AOGCM & $\begin{array}{c}\mathbf{F}_{2 \mathbf{x}} \\
\left(\mathbf{W m}^{\mathbf{2}}\right)\end{array}$ & $\begin{array}{c}\mathbf{T}_{\mathbf{2 x}} \\
\left({ }^{\mathbf{0}} \mathbf{C}\right)\end{array}$ & $\begin{array}{c}\Delta \mathbf{T}^{+} \\
\left.\mathbf{(}^{\mathbf{0}} \mathbf{C}\right)\end{array}$ & $\begin{array}{c}\mathbf{k} \\
\left(\mathbf{c m}^{\mathbf{2}} \mathbf{s}^{-\mathbf{1}}\right)\end{array}$ & $\mathbf{R L O}$ & $\begin{array}{c}\mathbf{L O} \text { and NS } \\
\left(\mathbf{W m}^{\mathbf{2}}{ }^{\circ} \mathbf{C}^{-\mathbf{1}}\right)\end{array}$ \\
\hline GFDL_R15_a & $3.71^{*}$ & 4.2 & 8 & 2.3 & 1.2 & 1.0 \\
\hline CSIRO Mk2 & 3.45 & 3.7 & 5 & 1.6 & 1.2 & 1.0 \\
\hline HadCM3 & 3.74 & 3.0 & 25 & 1.9 & 1.4 & 0.5 \\
\hline HadCM2 & 3.47 & 2.5 & 12 & 1.7 & 1.4 & 0.5 \\
\hline ECHAM4/OPYC & 3.8 & 2.6 & 20 & 9.0 & 1.4 & 0.5 \\
\hline CSM 1.0 & 3.60 & 1.9 & - & 2.3 & 1.4 & 0.5 \\
\hline DOE PCM & 3.60 & 1.7 & 14 & 2.3 & 1.4 & 0.5 \\
\hline
\end{tabular}

${ }^{*}$ Here the best estimate from Myhre et al. (1998) is used.

$\mathrm{F}_{2 \mathrm{x}} \quad$ - the radiative forcing for double $\mathrm{CO}_{2}$ concentration

$\mathrm{T}_{2 \mathrm{x}}$ - climate sensitivity

$\mathrm{hm} \quad$ - mixed-layer depth

CICE - sea ice parameter (see Raperet al., 2001a)

$\Delta \mathrm{T}^{+} \quad$ - magnitude of warming that would result in a collapse of the THC

$\mathrm{k}$ - vertical diffusivity

RLO - ratio of the equilibrium temperature changes over land versus ocean

LO and NS - land/ocean and Northern Hemisphere/Southern Hemisphere exchange coefficients 


\section{References}

Barthelet, P., L. Terray and S. Valcke, 1998a: Transient $\mathrm{CO}_{2}$ experimets using the ARPEGE/OPAICE non-flux corrected coupled model. Geophys. Res. Lett., 25, 2277-2280.

Barthelet, P., S. Bony, P. Braconnot, A. Braun, D. Cariolle, E. Cohen-Solal, J.-L. Dufresne, P. Delecluse, M. Déqué, L.Fairhead, M.-A., Filiberti, M. Forichon, J.-Y. Grandpeix, E. Guilyardi, M.-N. Houssais, M Imbard, H. LeTreut, C. Lévy, Z. X. Li, G. Madec, P. Marquet, O. Marti, S. Planton, L. Terray, O. Thual and S. Valcke, 1998b: Simulations couplées globales de changements climatiques associés à une augmentation de la teneur atmosphérique en $\mathrm{CO}_{2}$. C. R. Acad. Sci. Paris, Sciences de la terre et des planètes, 326, 677-684 (in French with English summary).

Bates, G.T. and G.A. Meehl, 1986: Effect of $\mathrm{CO}_{2}$ concentration on the frequency of blocking in a general circulation model coupled to a simple mixed layer ocean model. Mon. Wea. Rev., 114, 687-701.

Beersma, J.J., K.M. Rider, G.J. Komen, E. Kaas and V.V. Kharin, 1997: An analysis of extratropical storms in the North Atlantic region as simulated in a control and $2 \times \mathrm{CO}_{2}$ time-slice experiment with a highresolution atmospheric model. Tellus, 49A, 347-361.

Bengtsson, L., M. Botzet and M. Esch, 1996. Will greenhouse gas-induced warming over the next 50 years lead to a higher frequency and greater intensity of hurricanes? Tellus, 48A, 175-196.

Bhaskharan B. and Mitchell J.F.B., 1998: Simulated changes in the intensity and variability of the southeast Asian monsoon in the twenty first century resulting from anthropogenic emissions scenarios. Int. J. Climatol., 18, 1455-1462.

Boer, G.J., K. Arpe, M. Blackburn, M. Deque, W.L. Gates, T.L. Hart, H. le Treut, H. E. Roeckner, D.A. Sheinin, I. Simmonds, R.N.B. Smith, T. Tokioka, R.T. Wetherald and D. Williamson, 1992: Some results from an intercomparison of climates simulated by 14 atmospheric general circulation models. J. Geophys. Res., 97, 12,771-12,786.

Boer, G.J., G. Flato, M. C. Reader and D. Ramsden, 2000a: A transient climate change simulation with greenhouse gas and aerosol forcing: experimental design and comparison with the instrumental record for the 20th century. Clim. Dyn. 16, 405-425.

Boer, G.J., G. Flato, and D. Ramsden, 2000b: A transient climate change simulation with greenhouse gas and aerosol forcing: projected climate for the 21st century. Clim. Dyn. 16, 427-450.

Boville, B.A., J.T. Kiehl, P.J. Rasch and F.O. Bryan, 2001: Improvements to the NCAR-CSM-1 for transient climate simulations. J. Climate, 14, 164-179.

Brankovic, C. and T. Palmer, 2000: Seasonal skill and predictability of ECMWF PROVOST ensemble, Quart. J. R. Met. Soc., 126, 20352069.

Brinkop, S., 2001: Change of convective activity and extreme events in a transient climate change simulation, DLR-Institut fuer Physik der Atmosphaere, Report No. 142, [Available from DLROberpfaffenhofen, Institut fuer Physik der Atmosphaere, D-82234 Wessling, Germany].

Bryan, F. 1987: Parameter sensitivity of primitive equation ocean general circulation model. J. Phys. Oceanogr., 17. 970-985.

Buishand, T.A. and J.J. Beersma, 1996: Statistical tests for comparison of daily variability in observed and simulated climates. J. Climate, $\mathbf{9}$, 2538-2550

Cai, W. and P.H. Whetton, 2000: Evidence for a time-varying pattern of greenhouse warming in the Pacific Ocean. Geophys. Res. Lett., 27, 2577-2580

Cane M.A, A.C. Clement, A. Kaplan, Y. Kushnir, D. Pozdnyakov, R. Seager, S.E. Zebiak and R. Murtugudde, 1997: Twentieth century sea surface temperature trends. Science, 275, 957-960.

Carnell, R.E., C.A. Senior and J.F.B. Mitchell, 1996: An assessment of measures of storminess: simulated changes in Northern Hemisphere winter due to increasing $\mathrm{CO}_{2}$. Clim. Dyn., 12, 467-476.

Carnell, R.E. and C.A. Senior, 1998. Changes in mid-latitude variability due to increasing greenhouse gases and sulphate aerosols. Clim. Dyn., 14, 369-383.
Carnell, R.E. and C.A. Senior, 2000: Mechanisms of changes in storm tracks with increased greenhouse gases. Hadley Centre Technical Note 18. Available from Met Office, London Road Bracknell, RG12 2SZ, UK.

Christoph, M., U. Ulbrich and P. Speth, 1997: Midwinter suppression of Northern Hemisphere storm track activity in the real atmosphere and in GCM experiments. J. Atmos. Sci., 54, 1589-1599.

Collins, M., 2000a: The El-Niño Southern Oscillation in the second Hadley Centre coupled model and its response to greenhouse warming. $J$. Climate, 13, 1299-1312.

Collins, M., 2000b: Understanding uncertainties in the response of ENSO to greenhouse warming. Geophys. Res. Lett., 27, 3509-3512.

Colman, R.A. and B.J. McAvaney, 1995: Sensitivity of the climate response of an atmospheric general circulation model to changes in convective parameterisation and horizontal resolution. J. Geophys. Res. 100, 3155-3172.

Colman, R.A., 2001: On the vertical extent of GCM feedbacks. Clim. Dyn., in press.

Corti, S., F. Molteni, T.N. Palmer, 1999: Signature of recent climate change in frequencies of natural atmospheric circulation regimes. Nature, 398 , 799-802.

Cox, P.M., R.A. Betts, C.D. Jones, S.A. Spall, and I.J. Totterdell, 2000: Acceleration of global warming by carbon cycle feedbacks in a 3D coupled model. Nature, 408, 184-187.

Cubasch, U., K. Hasselmann, H. Höck, E. Maier-Reimer, U. Mikolajewicz, B. D. Santer and R. Sausen, 1992: Time-dependent greenhouse warming - computations with a coupled ocean-atmosphere model. Clim. Dyn., 8, 55-69.

Cubasch, U., B. D. Santer, A. Hellbach, G. Hegerl, H. Höck, E. MaierReimer, U. Mikolajewicz, A. Stössel and R. Voss, 1994: Monte Carlo climate change forecasts with a global coupled ocean-atmosphere model. Clim. Dyn., 10, 1-19.

Cubasch, U., G. C. Hegerl and J. Waszkewitz, 1996: Prediction, detection and regional assessment of anthropogenic climate change. Geophysica, 32, 77-96.

Cubasch, U., M. Allen, P. Barthelet, M. Beniston, C.Bertrand, S. Brinkop, J.-Y.Caneill, J.-L. Dufresne, L. Fairhead, M.-A. Filiberti, J. Gregory, G. Hegerl, G. Hoffmann, T. Johns, G. Jones, C. Laurent, R. McDonald, J. Mitchell, D. Parker, J. Oberhuber, C. Poncin, R. Sausen, U. Schlese, P. Stott, L. Terray, S. Tett, H. leTreut, U. Ulbrich, S. Valcke, R. Voss, M. Wild, J.-P. van Ypersele, 1999: Summary Report of the Project Simulation, Diagnosis and Detection of the Anthropogenic Climate Change (SIDDACLICH), EU-Commission, Brussels, EUR 19310, ISBN 92-828-8864-9.

Cubasch, U. and I. Fischer-Bruns, 2000: An intercomparison of scenario simulations performed with different AOGCMs, in: RegClim, General Technical Report No. 4, DNMI (Norwegian Meteorological Institute), eds. T. Iversen and B.A.K. Hoiskar.

Dai, A., T.M.L. Wigley, B. A. Boville, J.T. Kiehl, and L.E. Buja, 2001: Climates of the 20th and 21st centuries simulated by the NCAR climate system model. J. Climate, 14, 485-519.

Delworth, T.L., J.D. Mahlman, and T.R. Knutson: 2000: Changes in heat index associated with $\mathrm{CO}_{2}$-induced global warming. Clim. Change, $\mathbf{4 3}$, 369-386.

Derome, J., G. Brunet, A. Plante, N. Gagnon, G.J. Boer, F. Zwiers, S.Lambert, J. Sheng and H. Ritchie, 2001: Seasonal prediction based on two dynamical models, Atmos.-Ocean, in press.

Dessens, J., 1995: Severe convective weather in the context of a night-time global warming. Geophys. Res. Lett., 22, 1241-1244.

Dixon, K.W. and J.R. Lanzante, 1999: Global mean surface air temperature and North Atlantic overturning in a suite of coupled GCM climate change experiments. Geophys. Res. Lett., 26, 1885-1888.

Dixon, K. W., T. L. Delworth, M. J. Spelman and R. J. Stouffer, 1999: The influence of transient surface fluxes on North Atlantic overturning in a coupled GCM climate change experiment, Geophys. Res. Lett., 26, 2749-2752.

Doblas-Reyes, J., M. Deque and J.-P. Piedelievre, 2000: Multi-model spread and probabilistic seasonal forecasts in PROVOST, Quart. J. R. 
Met. Soc., 126, 2069 - 2089

Durman, C.F., J.M. Gregory, D.C. Hassell, R.G. Jones and J.M. Murphy, 2001: A comparison of extreme European daily precipitation simulated by a global and a regional model for present and future climates. Quart. J. R. Met. Soc., in press.

Dutton, J.F., C.J. Poulsen, and J.L. Evans, 2000: The effect of global climate change on the regions of tropical convection in CSM1. Geophys. Res. Lett., 27, 3049-3052.

Easterling, D.R., G. A. Meehl, C. Parmesan, S.A. Changnon, T.R. Karl and L.O. Mearns, 2000: Climate extremes: observations, modelling and impacts. Science, 289, 2068-2074.

Emori, S., T. Nozawa, A. Abe-Ouchi, A. Numaguti and M. Kimoto, 1999: Coupled ocean-atmosphere model experiments of future climate change with an explicit representation of sulphate aerosol scattering. $J$. Met. Soc. Japan, 77, 1299-1307.

Enting, I.G., T.M.L. Wigley and M. Heimann, 1994: Future emissions and concentrations of carbon dioxide: key ocean/atmosphere/land analyses, CSIRO Division of Atmospheric Research Technical Paper No. 31

Evans, J.L. and T. Kempisty, 1998: Tropical cyclone signatures in the climate. AMS Symposium on Tropical Cyclone Intensity Change, 1116 January, 1998, Phoenix AZ.

Fanning, A.F. and A.J. Weaver, 1996: An atmospheric energy-moisture balance model: climatology, interpentadal climate change, and coupling to an ocean general circulation model. J. Geophys. Res., 101, $15,111-15,128$.

Fanning, A.F. and A.J. Weaver, 1997: On the role of flux adjustments in an idealised coupled climate model. Clim. Dyn., 13, 691-701.

Flato, G.M. and G.J. Boer, 2001: Warming asymmetry in climate change simulations. Geophys. Res. Lett., 28, 195-198.

Friedlingstein, P., L. Bopp, P. Ciais, J.-L. Dufresne, L. Fairhead, H. LeTreut, P. Monfray, and J. Orr, 2001: Positive feedback of the carbon cycle on future climate change. Geophys. Res. Lett., in press.

Fyfe, J.C., 1999: On climate simulations of African easterly waves. J. Climate, 12, 1747-1769.

Fyfe, J.C., G.J. Boer, and G.M. Flato, 1999: The Arctic and Antarctic oscillations and their projected changes under global warming, Geophys. Res. Lett., 26, 1601-1604.

Ganopolski, A., V. Petoukhov, S. Rahmstorf, V. Brovkin, M. Claussen, A. Eliseev and C. Kubatzki, 2001: CLIMBER-2: A climate system model of intermediate complexity. Part II: validation and sensitivity tests. Clim. Dyn., in press.

Gates, W.L., J.S. Boyle, C.Covey, C.G. Dease, C.M. Doutriaux, R.S Drach, M. Fiorino, P.J. Gleckler, J.J. Hnilo, S.M. Marlais, T.J. Phillips, G.L. Potter, B.D. Santer, K.R. Sperber, K.E.Taylor and D.N. Williams, 1999: An overview of the results of the atmospheric model intercomparison project (AMIP I). Bull. Am. Met. Soc., 80, 29-55.

Gent, P.R., 2001: Will the North Atlantic Ocean thermohaline circulation weaken during the 21st century? Geophys. Res. Lett., in press.

Gordon, H.B. and S.P. O'Farrell, 1997: Transient climate change in the CSIRO coupled model with dynamic sea ice. Mon. Wea. Rev., 125, 875907.

Graham, R.J., A.D.L. Evans, K.R. Mylen, M.S.J. Harrison and K.B. Robertson, 1999: An assessment of seasonal predictability using atmospheric general circulation models. Forecasting Research Scientific Paper No. 54. UK Met Office, Bracknell Berkshire RG12 2SY, UK, $22 \mathrm{pp}$

Gray, W. M., 1979: Hurricanes: their formation, structure and likely role in the tropical circulation, In: Meteorology Over the Tropical Oceans, D.B. Shaw (ed), Royal Meteorological Society, J. Glaisher House, Grenville Place, Bracknell, Berks, pp. 155-218.

Gregory, J. M. and J. F. B. Mitchell, 1995: Simulation of daily variability of surface temperature and precipitation over Europe in the current and $2 \times \mathrm{CO}_{2}$ climate using the UKMO high-resolution climate model. Quart. J. R. Met. Soc., 121, 1451-1476.

Gregory, J.M. and J.F.B. Mitchell, 1997: The climate response to $\mathrm{CO}_{2}$ of the Hadley Centre coupled AOGCM with and without flux adjustment. Geophys. Res. Lett., 24, 1943-1946.

Gregory, J.M., J.F.B. Mitchell and A.J. Brady, 1997: Summer drought in
Northern midlatitudes in a time-dependent $\mathrm{CO}_{2}$ climate experiment. $J$. Climate, 10, 662-686.

Gregory, J.M. and J.A. Lowe, 2000: Predictions of global and regional sealevel rise using AOGCMs with and without flux adjustment. Geophys. Res. Lett., 27, 3069-3072.

Hansen, J., A. Lacis, D. Rind, G. Russell, P. Stone, I. Fung, R. Ruedy and J.Lerner, 1984: Climate sensitivity: analysis of feedback mechanisms, Met. Monograph, 29, 130-163.

Hansen, J., G. Russell, A. Lacis, I. Fung, D. Rind and P. Stone, 1985: Climate response times: dependence on climate sensitivity and ocean mixing. Science, 299, 857-859.

Harvey, D., J. Gregory, M. Hoffert, A. Jain, M. Lal, R. Leemans, S. Raper T. Wigley and J. de Wolde, 1997: An introduction to simple climate models used in the IPCC Second Assessment Report. [J.T. Houghton, L. G. Meira Filho, D. J. Griggs and K. Maskell (eds.)] IPCC Technical Paper II.

Haywood, J.M., R.J. Stouffer, R.T. Wetherald, S. Manabe and V. Ramaswamy, 1997: Transient response of a coupled model to estimated changes in greenhouse gas and sulphate concentrations. Geophys. Res. Lett., 24, 1335-1338.

Henderson-Sellers, A., H. Zhang, G. Berz, K. Emanuel, W. Gray, C. Landsea, G. Holland, J. Lighthill, S.-L. Shieh, P. Webster and K. McGuffie, 1998. Tropical cyclones and global climate change: a post IPCC assessment. Bull. Am. Met. Soc., 79, 19-38.

Hennessy, K.J., J.M. Gregory and J.F.B. Mitchell, 1997: Changes in daily precipitation under enhanced greenhouse conditions: comparison of UKHI and CSIRO9 GCM. Clim. Dyn., 13, 667-680.

Hirst, A.C. 1999: The Southern Ocean response to global warming in the CSIRO coupled ocean-atmosphere model. Environmental Modelling and Software, 14, 227-241.

Holland, G.J., 1997: Maximum potential intensity of tropical cyclones. J. Atmos. Sci., 54, 2519-2541.

Hu, Z.-Z., M. Latif, E. Roeckner and L. Bengtsson, 2000a: Intensified Asian summer monsoon and its variability in a coupled model forced by increasing greenhouse gas concentrations. Geophys. Res. Lett., 27, 2681-2684

Hu, Z.-Z., L. Bengtsson and K. Arpe, 2000b: Impact of the global warming on the Asian winter monsoon in a coupled GCM. J. Geophys. Res., 105, 4607-4624.

Hu, Z.-Z., L. Bengtsson, E. Roeckner, M. Christoph, A. Bacher and J. Oberhuber, 2001: Impact of global warming on the interannual and interdecadal climate modes in a coupled GCM. Clim. Dyn., in press.

Hurrell J.W., 1995: Decadal trends in the North Atlantic Oscillation: regional temperatures and precipitation. Science, 269, 676-679.

Hurrell J.W., 1996: Influence of variations in extratropical wintertime teleconnections on Northern Hemisphere temperature. Geophys. Res. Lett., 23, 1665-1668.

IPCC, 1990: Climate Change: The IPCC Scientific Assessment. Contribution of Working Group I to the First Assessment Report of the Intergovernmental Panel on Climate Change. [Houghton, J.T., G.J. Jenkins and J.J. Ephraums (eds.)]. Cambridge University Press, Cambridge, United Kingdom and New York, NY, USA, 365 pp.

IPCC, 1992: Climate Change 1992: The Supplementary Report to the IPCC Scientific Assessment. Report prepared for IPCC by Working Group I. [Houghton, J.T., B.A.Callander and S.K.Varney (eds.)]. Cambridge University Press, Cambridge, United Kingdom and New York, NY, USA, 200 pp.

IPCC, 1996: Climate Change 1995: The Science of Climate Change. Contribution of Working Group I to the Second Assessment Report of the Intergovernmental Panel on Climate Change. [Houghton, J.T., L.G. Meira Filho, B.A. Callander, N. Harris, A. Kattenberg, and K. Maskell (eds.)]. Cambridge University Press, Cambridge, United Kingdom and New York, NY, USA, 572 pp.

Japan Meteorological Agency, 1999: Information of Global Warming, Vol. 3 -Climate change due to increase of $\mathrm{CO}_{2}$ and sulphate aerosol projected with a coupled atmosphere ocean model (in Japanese). 70pp. (CD-ROM data are available from JMA.)

Jones, P.D., 1994: Hemispheric surface air temperature variations: a 
reanalysis and an update to 1993. J. Climate, 7, 1794-1802.

Johns, T.C., J.M. Gregory, W.J. Ingram, C.E. Johnson, A. Jones, J.A. Lowe, J.F.B. Mitchell, D.L. Roberts, D.M.H. Sexton, D.S. Stevenson, S.F.B Tett and M.J. Woodge, 2001: Anthropogenic climate change for 1860 to 2100 simulated with the HadCM3 model under updated emissions scenarios. Hadley Centre Technical Note No. 22, available from The Hadley Centre for Climate Prediction and Research, The Met Office, London Road, Bracknell, RG12 2SY, UK.

Joos, F., M. Bruno, R. Fink, T.F.Stocker, U. Siegenthaler, C. Le Quéré and J.L. Sarmiento, 1996: An efficient and accurate representation of complex oceanic and biospheric models of anthropogenic carbon uptake. Tellus, 48B, 397-417.

Kattenberg, A., F. Giorgi , H. Grassl, G.A. Meehl, J.F.B. Mitchell, R.J. Stouffer, T. Tokioka, A.J. Weaver and T.M.L.Wigley, 1996. In: Climate Change 1995: The Science of Climate Change. Contribution of Working Group I to the Second Assessment Report of the Intergovernmental Panel on Climate Change [Houghton, J.T., L.G. Meira Filho, B.A. Callander, N. Harris, A. Kattenberg, and K. Maskell (eds.)]. Cambridge University Press, Cambridge, United Kingdom and New York, NY, USA, 572 pp.

Keen, A.B. and J.M. Murphy, 1997: Influence of natural variability and the cold start problem on the simulated transient response to increasing $\mathrm{CO}_{2}$ Clim. Dyn. , 13, 847-864.

Kharin, V.V. and F.W. Zwiers, 2000: Changes in the extremes in an ensemble of transient climate simulations with a coupled atmosphereocean GCM. J. Climate, 13, 3760-3788.

Kitoh, A., H. Koide, K. Kodera, S. Yukimoto and A. Noda, 1996: Interannual variability in the stratospheric-tropospheric circulation in an ocean-atmosphere coupled GCM. Geophys. Res. Lett., 23, 543-546.

Kitoh, A., S. Yukimoto, A. Noda and T. Motoi, 1997. Simulated changes in the Asian summer monsoon at times of increased atmospheric $\mathrm{CO}_{2}$. Journal of the Meteorological Society of Japan, 75, 1019-1031.

Knippertz, P., U. Ulbrich and P. Speth, 2000: Changing cyclones and surface wind speeds over the North Atlantic and Europe in a transient GHG experiment. Clim. Res., 15, 109-122.

Knutson T.R. and S. Manabe, 1994: Impact of increased $\mathrm{CO}_{2}$ on simulated ENSO-like phenomena. Geophys. Res. Lett., 21, 2295-2298.

Knutson, T.R., and S. Manabe, 1995: Time-mean response over the tropical Pacific to increased $\mathrm{CO}_{2}$ in a coupled ocean-atmosphere model. $J$. Climate, 8, 2181-2199.

Knutson, T.R., S. Manabe and D. Gu, 1997: Simulated ENSO in a global coupled ocean-atmosphere model: multidecadal amplitude modulation and $\mathrm{CO}_{2}$-sensitivity. J. Climate, 10, 138-161.

Knutson, T.R. and S. Manabe, 1998: Model assessment of decadal variability and trends in the tropical Pacific ocean. J. Climate, 11, 22732296.

Knutson, T.R., R.E. Tuleya and Y. Kurihara, 1998: Simulated increase of hurricane intensities in a $\mathrm{CO}_{2}$-warmed climate. Science, 279, 10181020.

Knutson, T.R., T.L. Delworth, K.W. Dixon and R.J. Stouffer, 1999: Model assessment of regional surface temperature trends (1949-97). J. Geophys. Res., 104, 30,981-30,996.

Kodera, K., M. Chiba, H. Koide, A. Kitoh and Y. Nikaidou, 1996. Interannual variability of the winter stratosphere and troposphere in the Northern Hemisphere. Journal of the Meteorological Society of Japan, 74, 365-382.

Kothavala, Z., 1997: Extreme precipitation events and the applicability of global climate models to study floods and droughts. Math. and Comp. in Simulation, 43, 261-268.

Kothavala, Z., 1999: The duration and severity of drought over eastern Australia simulated by a coupled ocean-atmosphere GCM with a transient increase in $\mathrm{CO}_{2}$. Environmental Modelling Software, 14, 243 252

Krishnamurti, T.N., R. Correa-Torres, M. Latif and G. Daughenbaugh, 1998. The impact of current and possibly future SST anomalies on the frequency of Atlantic hurricanes. Tellus, 50A, 186-210.

Krishnamurti, T.N., C.M. Kishtawal, T.E. LaRow, D.R. Bachiochi, Z Zhang, C.E. Williford, S. Gadgil and S. Surendran, 1999: Improved weather and seasonal climate forecasts from multimodel superensemble. Science, 285, 1548-1550.

Kushner, P.J., I.M. Held and T.L. Delworth, 2001: Southern-hemisphere atmospheric circulation response to global warming. J. Climate, in press.

Lal, M., U. Cubasch, R. Voss and J. Waszkewitz, 1995: The effect of transient increase of greenhouse gases and sulphate aerosols on monsoon climate. Curr. Sci., 69, 752-763.

Lal, M., G.A. Meehl and J.M. Arblaster, 2000: Simulation of Indian summer monsoon rainfall and its intraseasonal variability. Regional Environmental Change, in press.

Lambert, S.J. and G.J. Boer, 2001: CMIP1 evaluation and intercomparison of coupled climate models. Clim. Dyn., 17, 83-106.

Latif, M., E. Roeckner, U. Mikolajewicz and R. Voss, 2000: Tropical stabilisation of the thermohaline circulation in a greenhouse warming simulation. J.Climate, 13, 1809-1813.

LeTreut, H. and B.J. McAvaney, 2000: A model intercomparison of equilibrium climate change in response to $\mathrm{CO}_{2}$ doubling. Note du Pole de Modelisation de l'IPSL, Number 18, Institut Pierre Simon LaPlace, Paris, France.

Lunkeit, F., M. Ponater, R. Sausen, M. Sogalla, U. Ulbrich and M. Windelband, 1996: Cyclonic activity in a warmer climate. Contrib. Atmos. Phys., 69, 393-407.

Lunkeit, F., S.E. Bauer and K. Fraedrich, 1998: Storm tracks in a warmer climate: sensitivity studies with a simplified global circulation model. Clim. Dyn., 14, 813-826.

Lupo, A.R., R.J. Oglesby and I.I. Mokhov, 1997: Climatological features of blocking anticyclones: a study of Northern Hemisphere CCM1 model blocking events in present-day and double $\mathrm{CO}_{2}$ concentrations. Clim. Dyn., 13, 181-195.

Manabe, S., R.J. Stouffer, M.J. Spelman and K. Bryan, 1991: Transient responses of a coupled ocean-atmosphere model to gradual changes of atmospheric $\mathrm{CO}_{2}$. Part I: annual mean response. J. Climate, 4, 785-818.

Manabe, S. and R.J. Stouffer, 1994: Multiple-century response of a coupled ocean-atmosphere model to an increase of the atmospheric carbon dioxide. J. Climate, 7, 5-23.

Matyasovszky,I., 1998: Non-parametric estimation of climate trends. Quarterly Journal of the Hungarian Meteorolgical Service, 102, 149158.

Meehl, G.A., G.W. Branstator and W.M. Washington, 1993: Tropical Pacific interannual variability and $\mathrm{CO}_{2}$ climate change. J. Climate, 6 , 42-63.

Meehl, G. A., M. Wheeler and W.M. Washington, 1994: Low-frequency variability and $\mathrm{CO}_{2}$ transient climate change. Part 3. Intermonthly and interannual variability. Clim. Dyn. 10, 277-303.

Meehl, G.A., W.M. Washington, D.J. Erickson III, B.P. Briegleb and P.J. Jaumann, 1996: Climate change from increased $\mathrm{CO}_{2}$ and direct and indirect effects of sulphate aerosols. Geophys. Res. Lett., 23, 3755-3758.

Meehl, G.A. and W.M. Washington, 1996: El Nino-like climate change in a model with increased atmospheric $\mathrm{CO}_{2}$-concentrations. Nature, $\mathbf{3 8 2}$, 56-60.

Meehl, G.A., G.J. Boer, C. Covey, M. Latif and R.J. Stouffer, 2000a: The Coupled Model Intercomparison Project (CMIP). Bull. Am. Met. Soc., 81, 313-318.

Meehl, G.A., W. Collins, B. Boville, J.T. Kiehl, T.M.L. Wigley and J.M. Arblaster, 2000b: Response of the NCAR Climate System Model to increased $\mathrm{CO}_{2}$ and the role of physical processes. J. Climate, 13, 18791898.

Meehl, G.A., W.M. Washington, J.M. Arblaster, T.W. Bettge and W.G. Strand Jr., 2000c: Anthropogenic forcing and decadal climate variability in sensitivity experiments of 20th and 21st century climate. J. Climate, 13, 3728-3744.

Meehl, G.A., F. Zwiers, J. Evans, T. Knutson, L. Mearns and P. Whetton, 2000d: Trends in extreme weather and climate events: issues related to modelling extremes in projections of future climate change. Bull. Am. Met. Soc., 81, 427-436.

Meehl, G.A., P. Gent, J.M. Arblaster, B. Otto-Bliesner, E. Brady and A. Craig, 2001: Factors that affect amplitude of El Nino in global coupled 
climate models. Clim. Dyn., 17, 515-526.

Mikolajewicz, U. and R. Voss, 2000: The role of the individual air-sea flux components in $\mathrm{CO}_{2}$-induced changes of the ocean's circulation and climate. Clim. Dyn. 16, 627-642.

Mitchell, J.F.B., S. Manabe, V. Meleshko and T. Tokioka, 1990. Equilibrium climate change - and its implications for the future. In Climate Change. The IPCC Scientific Assessment. Contribution of Working Group 1 to the first assessment report of the Intergovernmental Panel on Climate Change, [Houghton, J. L, G. J. Jenkins and J. J. Ephraums (eds)], Cambridge University Press, Cambridge, pp. 137-164.

Mitchell, J.F.B., T.C. Johns, J.M. Gregory and S.F.B. Tett, 1995: Climate response to increasing levels of greenhouse gases and sulphate aerosols. Nature, 376, 501-504.

Mitchell, J.F.B. and T.C. Johns, 1997: On the modification of global warming by sulphate aerosols. J. Climate, 10, 245-267.

Mitchell J.F.B., T.C. Johns and C.A. Senior, 1998: Transient response to increasing greenhouse gases using models with and without flux adjustment. Hadley Centre Technical Note 2. Available from Met Office, London Road Bracknell, RG12 2SZ, UK.

Mitchell, J.F.B., T.C. Johns, W.J. Ingram and J.A. Lowe, 2000: The effect of stabilising atmospheric carbon dioxide concentrations on global and regional climate change. Geophys. Res. Lett. 27, 2977-2930.

Monahan, A.H., J.C. Fyfe and G.M. Flato, 2000: A regime view of Northern Hemisphere atmospheric variability and change under global warming. Geophys. Res. Lett, 27, 1139-1142.

Murphy, J.M., 1995: Transient response of the Hadley Centre coupled ocean-atmosphere model to increasing carbon dioxide. Part III: analysis of global-mean response using simple models. J. Climate, 8, 496-514.

Murphy, J.M. and J.F.B. Mitchell, 1995: Transient response of the Hadley Centre coupled ocean-atmosphere model to increasing carbon dioxide. Part II: spatial and temporal structure of response. J. Climate, 8, 57-80.

Myhre, G., E.J. Highwood, K.P. Shine and F. Stordal, 1998: New estimates of radiative forcing due to well mixed greenhouse gases. Geophys. Res. Lett., 25, 2715-2718.

Nakićenović, N., J. Alcamo, G. Davis, B. de Vries, J. Fenhann, S. Gaffin, K. Gregory, A. Grübler, T. Y. Jung, T. Kram, E. L. La Rovere, L. Michaelis, S. Mori, T. Morita, W. Pepper, H. Pitcher, L. Price, K. Raihi, A. Roehrl, H.-H. Rogner, A. Sankovski, M. Schlesinger, P. Shukla, S. Smith, R. Swart, S. van Rooijen, N. Victor, Z. Dadi, 2000: IPCC Special Report on Emissions Scenarios, Cambridge University Press, Cambridge, United Kingdom and New York, NY, USA, 599 pp.

Noda, A. and T. Tokioka, 1989: The effect of doubling the $\mathrm{CO}_{2}$ concentration on convective and non-convective precipitation in a general circulation model coupled with a simple mixed layer ocean model. J. Met. Soc. Japan, 67, 1057-1069.

Noda, A., K. Yoshimatsu, A. Kitoh and H. Koide, 1999a: Relationship between natural variability and $\mathrm{CO}_{2}$-induced warming pattern: MRI coupled atmosphere/mixed-layer (slab) ocean GCM (SGCM) Experiment. 10th Symposium on Global Change Studies , 10-15 January 1999, Dallas, Texas. pp. 355-358, American Meteorological Society, Boston. Mass.

Noda, A., K. Yoshimatsu, S. Yukimoto, K. Yamaguchi and S. Yamaki, 1999b: Relationship between natural variability and $\mathrm{CO}_{2}$-induced warming pattern: MRI AOGCM Experiment. 10th Symposium on Global Change Studies, 10-15 January 1999, Dallas, Texas. American Meteorological Society, Boston. Mass. pp. 359-362

Noda, A., S. Yukimoto, S. Maeda, T. Uchiyama, K. Shibata and S. Yamaki, 2001: A new meteorological research institute coupled GCM (MRICGCM2): Transient response to greenhouse gas and aerosol scenarios. CGER's supercomputer monograph report Vol. 7, National Institute for Environmental Studies, Tsukuba, Japan, 66pp (in press).

Nozawa, T., S. Emori, A. Numaguti, Y. Tsushima, T. Takemura, T. Nakajima, A. Abe-Ouchi and M. Kimoto, 2001: Projections of future climate change in the $21^{\text {st }}$ century simulated by the CCSR/NIES CGCM under the IPCC SRES scenarios, In: Present and Future of Modelling Global Environmental Change - Toward Integrated Modelling, T. Matsuno (ed), Terra Scientific Publishing Company, Tokyo (in press).
Osborn, T.J., K.R. Briffa, S.F.B. Tett, P.D. Jones and R.M. Trigo, 1999. Evaluation of the North Atlantic Oscillation as simulated by a coupled climate model. Clim. Dyn., 15, 685-702.

Paeth, H., A. Hense, R. Glowienka-Hense, R. Voss and U. Cubasch, 1999: The North Atlantic Oscillation as an indicator for greenhouse-gas induced climate change. Clim. Dyn., 15, 953-960.

Palmer, T. N., 1999. A nonlinear dynamical perspective on climate prediction. J. Climate, 12, 575-591.

Parey, S, 1994: Simulations de Trente ans $1 \mathrm{xCO}_{2}, 2 \mathrm{xCO}_{2}, 3 \mathrm{xCO}_{2}$, avec le modele du LMD (64x50x11) premiers resultata. EDF (Electricité de France), Direction des études et Recherches, HE-33/94/008.

Price, C., and D. Rind, 1994a: Possible implications of global climate change on global lightning distributions and frequencies. J. Geophys. Res., 99, 10,823-10,831.

Price, C., and D. Rind, 1994b: The impact of a $2 \mathrm{xCO}_{2}$ climate on lightningcaused fires. J. Climate, 7, 1484-1494.

Räisänen, J., 1997: Objective comparison of patterns of $\mathrm{CO}_{2}$-induced climate change in coupled GCM experiments. Clim. Dyn., 13, 197-221.

Räisänen, J., 2000: $\mathrm{CO}_{2}$-induced climate change in Northern Europe: comparison of 12 CMIP2 experiments. Reports Meteorology and Climatology No. 87, SMHI, 59 pp.

Räisänen, J., 2001: $\mathrm{CO}_{2}$-induced climate change in CMIP2 experiments. Quantification of agreement and role of internal variability. J. Climate, in press.

Ramstein, G., Y. Serafini-Le Treut, H. Le Treut, M. Forichon and S. Joussaume, 1998. Cloud processes associated with past and future climate changes. Clim. Dyn., 14, 233-247.

Raper, S.C.B, T.M.L. Wigley and R.A. Warrick, 1996: Global sea-level rise: past and future, In: Sea-Level rise and Coastal Subsidence, J.D. Milliman and B.U. Haq (eds), Kluwer Academic Publishers, 11-46.

Raper, S.C.B. and U. Cubasch, 1996: Emulation of the results from a coupled general circulation model using a simple climate model. Geophys. Res. Lett., 23, 1107-1110.

Raper, S.C.B., J.M. Gregory and T.J. Osborn, 2001a: Use of an upwellingdiffusion energy balance climate model to simulate and diagnose AOGCM results. Clim. Dyn., in press.

Raper, S.C.B., J.M. Gregory and R.J. Stouffer, 2001b: The role of climate sensitivity and ocean heat uptake on AOGCM transient temperature and thermal expansion response. J. Climate, in press.

Roeckner, E., L. Bengtsson, J. Feichter, J. Lelieveld and H. Rodhe, 1999: Transient climate change with a coupled atmosphere-ocean GCM including the tropospheric sulfur cycle. J. Climate, 12, 3004-3032.

Royer, J.-F., F. Chauvin, B. Timbal, P. Araspin and D. Grimal, 1998. A GCM study of the impact of greenhouse gas increase on the frequency of occurrence of tropical cyclones. Clim. Change, 38, 307-343.

Russell, G.L., J.R. Miller and D. Rind, 1995: A coupled atmosphere-ocean model for transient climate change studies. Atmosphere-Ocean, 33, $683-730$

Russell, G.L. and D. Rind, 1999. Response to $\mathrm{CO}_{2}$ transient increase in the GISS coupled model. Regional cooling in a warming climate. $J$. Climate, 12, 531-539.

Sarmiento, J.L., T.M.C. Hughes, R.J. Stouffer and S. Manabe, 1998: Simulated response of the ocean carbon cycle to anthropogenic climate warming. Nature, 393, 245-249.

Schimel, D., I.G. Enting, M. Heimann, T.M.L. Wigley, D. Raynaud, D. Alves and U. Siegenthaler, 1994. $\mathrm{CO}_{2}$ and the carbon cycle. In: Climate Change, 1994: Radiative Forcing of Climate Change and an Evaluation of the IPCC IS92 Emission Scenarios. [J.T. Houghton, L.G. Meira Filho, J. Bruce, H Lee, B.A. Callander, E.F. Haites, N. Harris and K. Maskell (eds.)] Cambridge University Press, Cambridge, UK, 35-71.

Schimel, D., M. Grubb, F. Joos, R. Kufmann, R. Moss, W. Ogana, R. Richels, T. Wigley , 1997: Stabilisation of Atmospheric Greenhouse Gases: Physical, Biological and Socio-economic Implications. [J.T. Houghton, L. Gylvan Meira Filho, D.J. Griggs and K. Maskell (eds.)] IPCC Technical Paper III.

Schubert, M., J. Perlwitz, R. Blender, K. Fraedrich, and F. Lunkeit, 1998: North Atlantic cyclones in $\mathrm{CO}_{2}$-induced warm climate simulations: Frequency, intensity, and tracks. Clim. Dyn. , 14, 827-837. 
Senior, C.A., 1999: Comparison of mechanisms of cloud-climate feedbacks in a GCM. J. Clim., 12, 1480-1489.

Senior, C.A. and J.F.B. Mitchell, 2000: The time-dependence of climate sensitivity. Geophys. Res. Lett., 27, 2685-2688.

Shindell, D.T., R.L. Miller, G.A. Schmidt and L. Pandolfo, 1999 Simulation of recent northern winter climate trends by greenhouse gas forcing. Nature, 399, 452-455.

Shine, K.P., R.G. Derwent, D.J. Wuebbles and J.-J.Morcrette, 1990: Radiative forcing of climate. In: Climate Change: The IPCC Scientific Assessment. [Houghton, J.T., G.J. Jenkins and J.J. Ephraums (eds.)]. Cambridge University Press, Cambridge, United Kingdom and New York, NY, USA, pp. 41-68.

Siegenthaler, U. and Joos, F., 1992: Use of a simple model for studying oceanic tracer distributions and the global carbon cycle. Tellus, 44B, 186-207.

Sinclair, M.R. and I.G. Watterson, 1999: Objective assessment of extratropical weather systems in simulated climates. J. Climate, 12, 34673485 .

Smith, S. J., T.M.L.Wigley, N. Nakićenović and S.C.B. Raper, 2001: Climate implications of greenhouse gas emission scenarios. Technological Forecasting and Social Change, 65, 195-204.

Sokolov, A.P. and P.H. Stone 1998: A flexible climate model for use in integrated assessments. Clim. Dyn., 14, 291-303.

Stendel, M., T. Schmith, E. Roeckner and U. Cubasch, 2000: The climate of the 21st century: transient simulations with a coupled atmosphereocean general circulation model. Danmarks Klimacenter Report 00-6, Danish Meteorological Institute, Lyngbyvej 100, DK-2100 Copenhagen, Denmark, ISBN: 87-7478-427-7.

Stocker T.F., D.G. Wright and L.A. Mysak, 1992: A zonally averaged, coupled ocean-atmosphere model for paleoclimate studies. J. Climate, 5, 773-797.

Stocker, T.F. and A. Schmittner, 1997: Influence of $\mathrm{CO}_{2}$ emission rates on the stability of the thermohaline circulation. Nature, 388, 862-865.

Stouffer, R.J. and S. Manabe, 1999: Response of a coupled oceanatmosphere model to increasing atmospheric carbon dioxide: Sensitivity to the rate of increase. J. Climate, 12, 2224-2237.

Tett, S.F.B., 1995: Simulation of El Niño-Southern Oscillation-like variability in a global coupled AOGCM and its response to $\mathrm{CO}_{2-}$ increase. J. Climate, 8, 1473-1502.

Tett, S.F.B., T.C. Johns and J.F.B. Mitchell, 1997: Global and regional variability in a coupled AOGCM. Clim. Dyn., 13, $303-323$.

Thompson, D.W.J. and J.M. Wallace, 1998: The Arctic Oscillation signature in the wintertime geopotential height and temperature fields. Geophys. Res. Lett., 25, 1297-1300.

Timmermann, A., J. Oberhuber, A. Bacher, M. Esch, M. Latif and E. Roeckner, 1999: Increased El Niño frequency in a climate model forced by future greenhouse warming. Nature, 398, 694-696.

Tokioka, T., A. Noda, A. Kitoh, Y. Nikaidou, S. Nakagawa, T. Motoi, Y. Yukimoto and K. Takata, 1995: Transient $\mathrm{CO}_{2}$ experiment with the MRI CGCM - Quick report. J. Met. Soc. Japan, 73, 817-826.

Tokioka, T., A. Noda, A. Kitoh, Y. Nikaidou, S. Nakagawa, T. Motoi, Y. Yukimoto and K. Takata, 1996: Transient $\mathrm{CO}_{2}$ experiment with the MRI CGCM - Annual mean response -. CGER's Supercomputer Monograph Report Vol. 2, National Institute for Environmental Studies, Tsukuba, Japan, 86 pp.

Ulbrich, U., and M. Christoph, 1999: A shift of the NAO and increasing storm track activity over Europe due to anthropogenic greenhouse gas forcing. Clim. Dyn., 15, 551-559.

United Nations, 1992: United Nations framework convention on climate change, UNFCCC. http://www.unfccc.int/

Visser, H., R.J.M. Folkert, J. Hoekstra and J.J.de Wolff, 2000: Identifying key sources of uncertainty in climate change projections. Clim. Change, 45, 421-457.

Voss, R. and U. Mikolajewicz, 2001: Long-term climate changes due to increased $\mathrm{CO}_{2}$ concentration in the coupled atmosphere-ocean general circulation model ECHAM3/LSG. Clim. Dyn., 17, 45-60.

Wallace, J. M., 2000. North Atlantic Oscillation / annular mode: Two paradigms - one phenomenon. Quart. J. R. Met. Soc, 126, 791-806.

Washington, W.M., J.W. Weatherly, G.A. Meehl, A.J. Semtner Jr., T.W. Bettge, A.P. Craig, W.G. Strand Jr., J.M. Arblaster, V.B. Wayland, R. James and Y. Zhang, 2000: Parallel climate model (PCM) control and transient simulations. Clim. Dyn., 16, 755-774.

Watterson, I. G., M.R. Dix and R.A. Colman, 1998: A comparison of present and doubled $\mathrm{CO}_{2}$ climates and feedbacks simulated by three general circulation models. J. Geophys. Res., 104, 1943-1956.

Watterson, I. G. 2000: Interpretation of simulated global warming using a simple model. J. Climate, 13, 202-215.

Weaver, A.J., M. Eby, A.F. Fanning and E.C. Wiebe, 1998: Simulated influence of carbon dioxide, orbital forcing and ice sheets on the climate of the last glacial maximum. Nature, 394, 847-853.

Weaver, A.J. and E.C. Wiebe, 1999: On the sensitivity of projected oceanic thermal expansion to the parameterisation of sub-grid scale ocean mixing. Geophys. Res. Lett., 26, 3461-3464.

Wetherald, R. T. and S. Manabe, 1999: Detectability of summer dryness caused by greenhouse warming. Climatic Change, 43, 495-511.

Wiebe, E.C. and A.J. Weaver, 2000: On the sensitivity of global warming experiments to the parameterisation of sub-grid scale ocean mixing. Clim. Dyn., 15, 875-893.

Wigley T.M.L. and Raper S.C.B., 1987: Thermal expansion of sea water associated with global warming. Nature, 330, 127-131.

Wigley, T.M.L., 1991: Could reducing fossil-fuel emissions cause global warming? Nature, 349, 503-506.

Wigley T.M.L. and S.C.B. Raper,1992: Implications for climate and sea level of revised IPCC emissions scenarios. Nature, 357, 293-300.

Wigley, T.M.L. 1993: Balancing the carbon budget. Implications for projections of future carbon dioxide concentration changes. Tellus, 45B, 409-425.

Wigley, T.M.L, R. Richels and J.A. Edmonds, 1996: Economic and environmental choices in the stabilisation of atmospheric $\mathrm{CO}_{2}$ concentrations. Nature, 379, 242-245.

Wigley, T.M.L. and S.J. Smith, 1998: Uncertainties in projections of future global-mean temperature change. In: Do We Understand Global Climate change? Norwegian Academy of Technological Sciences (NTVA), Trondheim, Norway, 185-195.

Wigley, T.M.L., 1999: The science of climate change: global and U.S. perspectives. Pew Centre, 2101 Wilson Blvd., Arlington, VA, USA, 48pp.

Wigley, T.M.L., 2000: TAR version of MAGICC forcing. http://www.acacia.ucar.edu

Williams, K. D., C.A. Senior and J.F.B. Mitchell, 2001. Transient climate change in the Hadley Centre models: The roles of physical processes. J. Climate, in press.

Yao, M-S. and Del Genio, A., 1999. Effects of parameterisation on the simulation of climate changes in the GISS GCM. J. Climate., 12, 761779 .

Yonetani, T. and H.B. Gordon, 2001: Simulated changes in the frequency of extremes and regional features of seasonal/annual temperature and precipitation when atmospheric $\mathrm{CO}_{2}$ is doubled. J.Climate, in press.

Yukimoto, S., A. Noda, A. Kitoh, M. Sugi, Y. Kitamura, M. Hosaka, K. Shibata, S. Maeda and T. Uchiyama, 2001: A new meteorological research institute coupled GCM (MRI-CGCM2) - model climate and its variability. Pap. Meteor. Geophys., 51, 47-88.

Zhang, Y. and W.-Ch. Wang, 1997: Model simulated northern winter cyclone and anti-cyclone activity under a greenhouse warming scenario. J. Climate, 10, 1616-1634.

Zhang, Y., J.M. Wallace and D.S. Battisti, 1997: ENSO-like interdecadal variability: 1900-93. J. Climate, 10, 1004-1020.

Zorita, E. and F. González-Rouco, 2000. Disagreement between predictions of the future Atrctic Oscillation as simulated in two different climate models: Implications for global warming. Geophys. Res. Lett., 27, 1755-1758

Zwiers, F.W. and V. V. Kharin, 1998: Changes in the extremes of the climate simulated by $\mathrm{CCC}$ GCM2 under $\mathrm{CO}_{2}$-doubling. J. Climate, $\mathbf{1 1}$, 2200-2222. 